\title{
Ceramographic Examinations of Irradiated AGR-1 Fuel Compacts
}

The INL is a

U.S. Department of Energy

National Laboratory

operated by

Battelle Energy Alliance

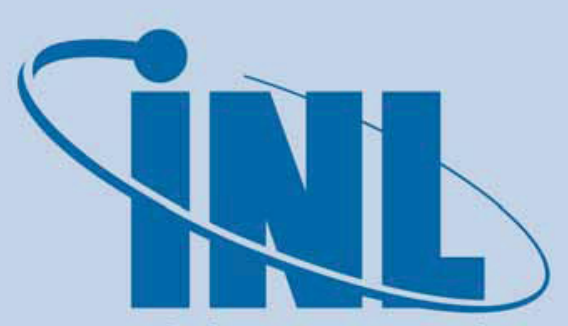

Idaho National Laboratory
Scott A. Ploger

Paul A. Demkowicz

John D. Hunn

May 2012

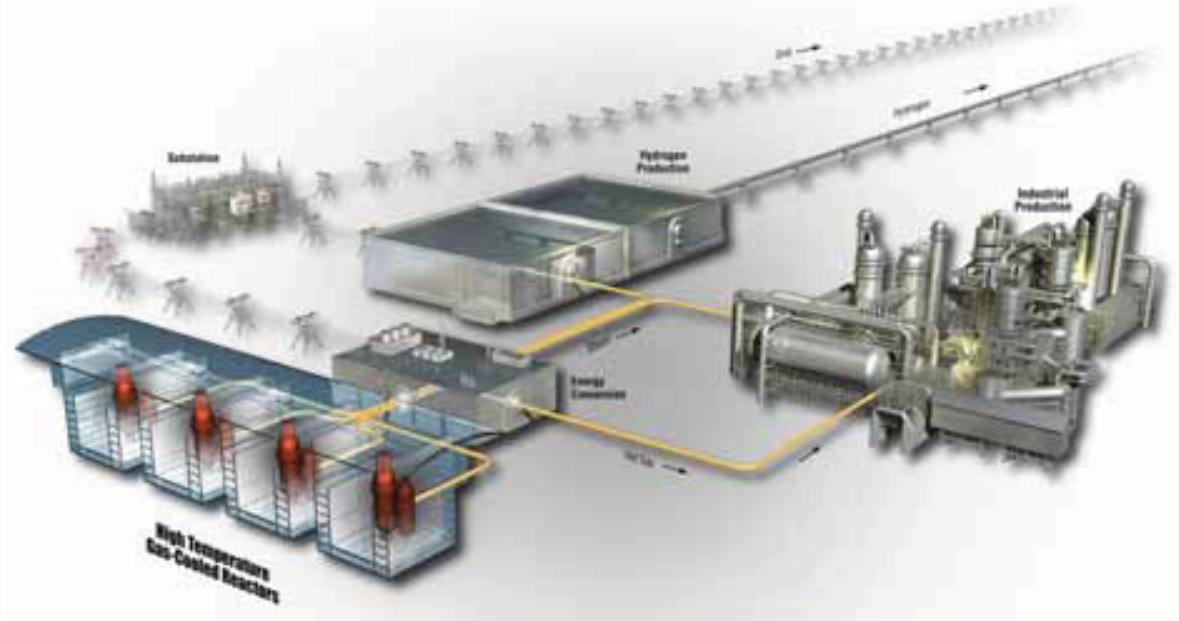




\section{DISCLAIMER}

This information was prepared as an account of work sponsored by an agency of the U.S. Government. Neither the U.S. Government nor any agency thereof, nor any of their employees, makes any warranty, expressed or implied, or assumes any legal liability or responsibility for the accuracy, completeness, or usefulness, of any information, apparatus, product, or process disclosed, or represents that its use would not infringe privately owned rights. References herein to any specific commercial product, process, or service by trade name, trade mark, manufacturer, or otherwise, does not necessarily constitute or imply its endorsement, recommendation, or favoring by the U.S. Government or any agency thereof. The views and opinions of authors expressed herein do not necessarily state or reflect those of the U.S. Government or any agency thereof. 


\title{
Ceramographic Examinations of Irradiated AGR-1 Fuel Compacts
}

\author{
Scott A. Ploger \\ Paul A. Demkowicz \\ John D. Hunn
}

May 2012

\begin{abstract}
Idaho National Laboratory
Very High Temperature Reactor Project

Idaho Falls, Idaho 83415
\end{abstract}

http://www.inl.gov

Prepared for the

U.S. Department of Energy

Office of Nuclear Energy

Under DOE Idaho Operations Office

Contract DE-AC07-05ID14517 



\section{Very High Temperature Reactor Project}

\section{Ceramographic Examinations of Irradiated AGR-1 Fuel Compacts}

INL/EXT-12-25301

May 2012

Approved by:

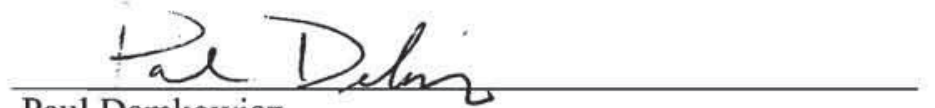

Paul Demkowicz

VHTR Fuels Post-irradiation Technical Lead

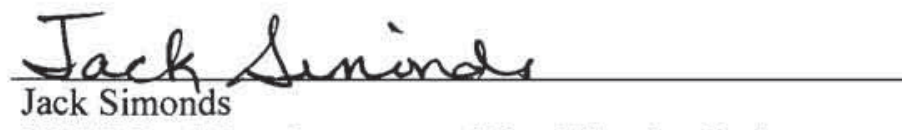

VHTR Fuel Development and Qualification Project

Manager

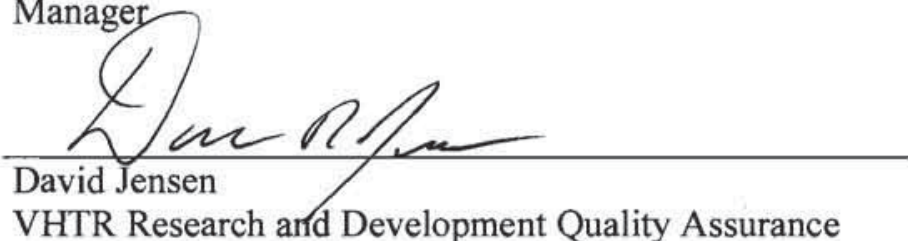

VHTR Research and Development Quality Assurance

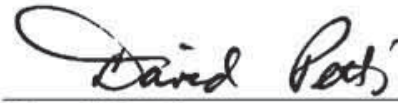

David Petti

VHTR Research and Development Director
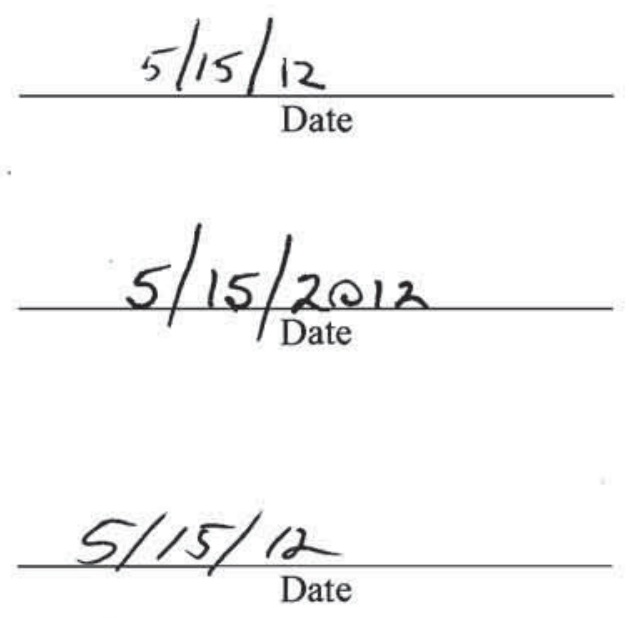

$5 / 15 / 12$ Date 



\section{SUMMARY}

The AGR-1 experiment involved irradiating 72 cylindrical fuel compacts containing tri-structural isotropic (TRISO)-coated particles to a peak burnup of $19.5 \%$ fissions per initial metal atom with no inpile failures observed out of almost 300,000 particles. Five irradiated AGR-1 fuel compacts were selected for microscopy that spanned the range of irradiation conditions (temperature, burnup, and fast fluence). These five compacts also included all four TRISO coating variations irradiated in the AGR-1 experiment. The five compacts were cross-sectioned both transversely and longitudinally, mounted, ground, and polished after development of careful techniques for preserving particle structures against preparation damage. Approximately 40 to 80 particles within each cross section were exposed near enough to midplane for optical microscopy of kernel, buffer, and coating behavior. The microstructural analysis focused on kernel swelling and porosity, buffer densification and fracturing, debonding between the buffer layer and the inner pyrolytic carbon (IPyC) layer, and fractures in the IPyC and SiC (silicon carbide) layers.

Three basic particle morphologies were established according to the extent of bonding between the buffer and IPyC layers: complete debonding along the interface (Type A), no debonding along the interface (Type B), and partial debonding (Type AB). These basic morphologies were subdivided according to whether the buffer stayed intact or fractured. The resulting six characteristic morphologies were used to classify particles within each cross section, but no spatial patterns were clearly observed in any of the cross-sectional morphology maps. Although positions of particle types appeared random within compacts, examining a total of 830 classified particles allowed several specific relationships among morphological types to be established:

- Buffer-IPyC debonding was prevalent in irradiated AGR-1 particles. Nearly $62 \%$ of the irradiated AGR-1 particles showed a radial gap between buffer and IPyC layers from complete debonding while less than $6 \%$ of the particles had fully intact buffer-IPyC bonds. Partial debonding was found in approximately $33 \%$ of the classified particles. These percentages varied only slightly among the cross-sectioned compacts, so differences in irradiation temperature and neutron exposure (burnup, fast fluence) apparently had little influence on buffer-IPyC bond integrity.

- Buffer layers remained intact in over three-fourths of the AGR-1 particles. Fractured buffers were nearly three times as likely in particles with partial buffer-IPyC debonding (Type AB) than in particles with complete debonding (Type A). This tendency was found in all five cross-sectioned compacts, so differences among them in irradiation temperature and neutron exposure apparently had little influence on buffer fracturing.

- Buffer fractures in Type A particles tended to have smooth radially oriented contours. Buffer fractures in Type $\mathrm{AB}$ particles tended to have jagged contours (mixture of tangential, circumferential, and radial directions). Most buffer fractures in Type $\mathrm{AB}$ particles appear to have initiated tangentially at the kernel interface, while most buffer fractures in Type A particles apparently initiated radially.

In addition to buffer fractures, all classified particles were inspected for fractures in other TRISO coating layers. No irradiation-induced fractures were found in OPyC layers. OPyC layers also resisted fracturing during the occasional cases where the carbonaceous compact matrix fractured when compacts were sawed and mounted. The only instances found of OPyC-matrix debonding were caused by preparation damage, especially sawing of the compacts.

Although partial IPyC fractures and more severe through-IPyC fractures were found in only 50 of the 830 particles $(6 \%)$, fractures through the entire IPyC layer were much more likely than average in particles from the Variant 1 compact, which had the lowest density IPyC layer. Significant variations in IPyC fracturing behavior also were seen among morphological types: 
- All buffer fractures in Type B particles propagated into IPyC fractures, presumably because of the intact buffer/IPyC interface.

- $\quad$ Through-IPyC fractures were more likely in particles with partial buffer-IPyC debonding (Type AB) than with complete debonding (Type A).

- In Type $\mathrm{AB}$ particles, IPyC fractures tended to occur where buffer-IPyC debonds ended (where buffer-IPyC bonding was locally strong).

- In Type $\mathrm{AB}$ particles, IPyC fractures were much more likely with fractured buffers than with intact buffers, although IPyC fractures in these particles normally did not propagate from buffer fractures.

Extra attention was paid to extremely rare $\mathrm{SiC}$ fractures because $\mathrm{SiC}$ constitutes the primary barrier to migration of metallic fission products in TRISO-coated particle fuel:

- Tangential SiC fractures were found in only five particles from the five cross-sectioned AGR-1 compacts. All of the observed fractures were apparently confined close to the IPyC interface, and none appeared to breach the entire layer thickness during the AGR-1 experiment.

- Two of these particles contained foreign material along a portion of the IPyC-SiC interface, which may be soot picked up from the coating chamber wall. The SiC fractures in these two particles initiated tangentially where the foreign material ended and IPyC was able to bond to the $\mathrm{SiC}$.

- Two Type B particles showed tangential SiC fractures at the ends of extensive IPyC-SiC debonds, where the debonding apparently began at the tips of radial fractures through both buffer and IPyC.

- One unclassified particle exhibited a tangential $\mathrm{SiC}$ fracture that may have propagated directly from a tangential IPyC fracture.

- Tangential $\mathrm{SiC}$ fractures appeared more likely in conjunction with extensive IPyC-SiC debonds. Very short IPyC-SiC debonds were found at tips of IPyC fractures in eight Type AB particles with fractured buffers, but no $\mathrm{SiC}$ fractures were discovered in these eight particles. SiC fractures also seemed somewhat more likely in particles from the Variant 1 compact, which probably is related to its much higher frequency of through-IPyC fractures.

Observations of kernels in the five AGR-1 compacts suggested that kernels often swelled into fractured buffer cavities, at least where buffer fractures occurred relatively early during irradiation. Kernel pores generally appeared somewhat larger inside fractured buffers than nearby intact buffers, perhaps reflecting less constraint on kernels after buffer fracturing. Similar side-by-side comparisons indicated that pores were even larger inside Type B kernels, where outward buffer densification imposed even less constraint on kernel growth.

Insights on kernel and buffer behavior were obtained by extracting measurements from a total of 95 calibrated particle images taken from three transverse cross sections, where the three compacts were selected to cover extremes of irradiation temperature and neutron exposure. To reduce geometric errors, only those particles ground close to midplane were measured. Movement of loose kernels and buffers inside IPyC layers compromised accurate measurements, but dimensions in the plane of polish were obtained and summarized to look for trends. The following observations could be made despite the wide distributions in measurement results:

- Cross-sectional kernel areas increased during the AGR-1 irradiation. Distributions were similar for all three compacts, so differences in irradiation temperatures and neutron exposures apparently had no major influence on amounts of kernel swelling.

- Although kernels often swelled into buffer fracture cavities, area increases in kernels surrounded by fractured buffers were similar to those inside intact buffers. This probably reflects constraint 
on kernel growth before buffers fractured. Buffer fractures apparently had more influence on where kernels swelled than on how much they swelled.

- Kernel area increases were slightly larger on average in Type B particles, where uniformly strong buffer-IPyC bonding and the associated outward buffer densification imposed the least constraint on kernel swelling over the course of the AGR-1 experiment.

- Porosity (area percentage inside kernel exteriors) and pore enlargement (as indicated by the diameter of the largest pore in each kernel) were greatest in Type B kernels with the least constraint on kernel swelling and smallest in Type A and Type AB kernels with intact buffers (maximum constraint on kernel swelling from inward buffer densification).

- All measured buffers showed appreciable decreases in area and thickness from as-fabricated values. No differences in the measured buffer parameters could be discerned among the three compacts. Buffer area and thickness reductions appeared similar for radially inward densification (Types A and $\mathrm{AB}$ ) and outward densification (Type B).

- Inward buffer densification created a thick buffer-IPyC gap in Type A and Type AB particles with intact buffers. Average gaps with fractured buffers were approximately one-half as thick, evidently because buffer pieces separated after fracturing and moved closer to IPyC interiors. 


\section{ACKNOWLEDGEMENTS}

Careful preparation of fuel compact cross sections for microscopic examinations by the skillful operators at the INL Hot Fuel Examination Facility was absolutely essential to the success of this effort. In particular, new techniques had to be developed to stabilize internal structures of AGR-1 fuel particles against damage during grinding. Choice of grinding disks, polishing cloths, and polishing suspensions also had to be painstakingly optimized. The cooperation, creative contributions, and patience of Brian Frickey, Cad Christensen, Colt Killian, and Lawrence Brower are gratefully acknowledged.

The authors also thank Dr. James Kendall (Global Virtual LLC) for his constructive contributions during formative discussions on particle classification, particularly regarding the importance of buffer$\mathrm{IPyC}$ bonding. 


\section{CONTENTS}

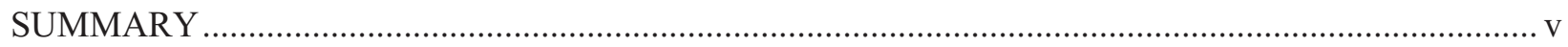

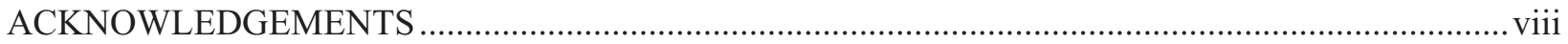

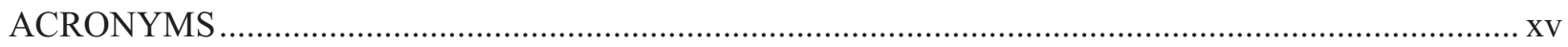

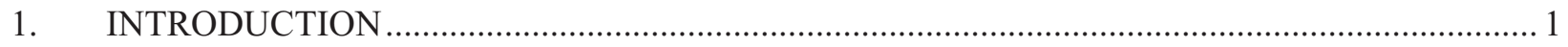

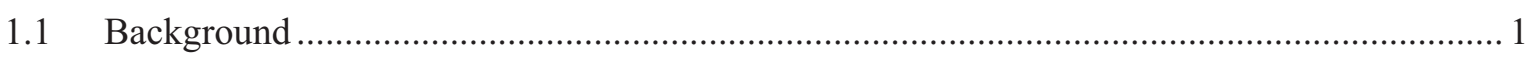

1.2 Objectives for AGR-1 Compact Ceramography …....................................................... 2

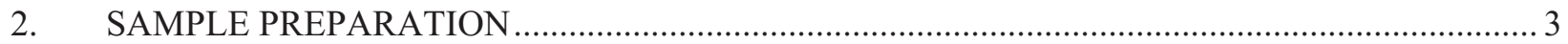

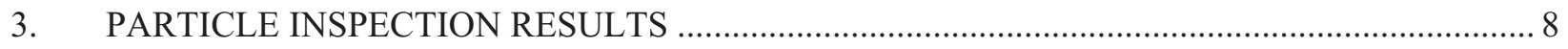

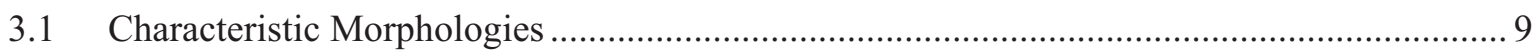

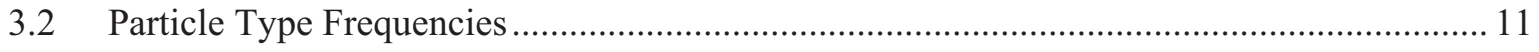

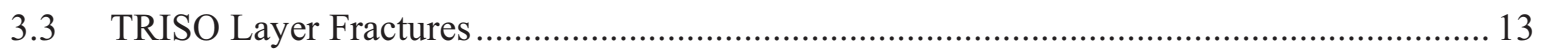

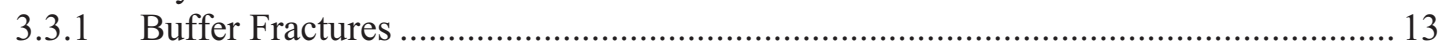

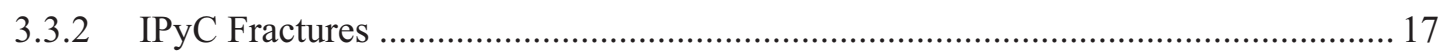

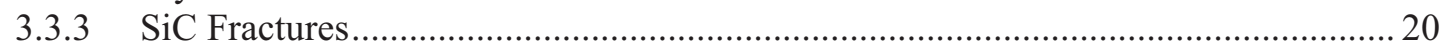

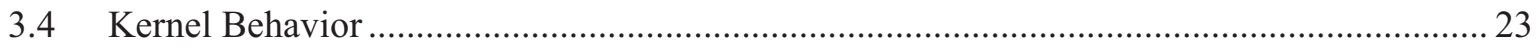

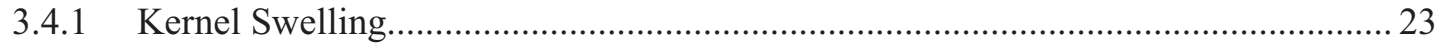

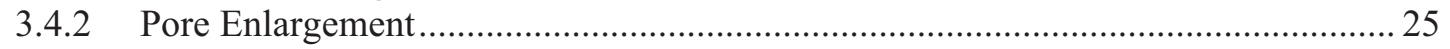

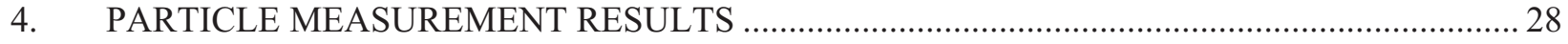

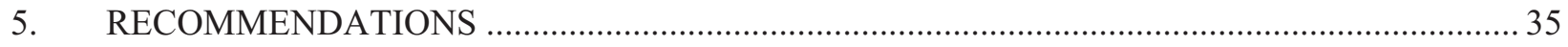

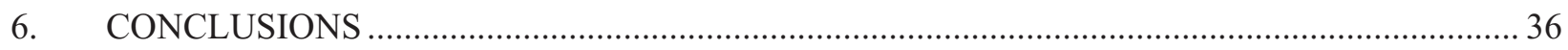

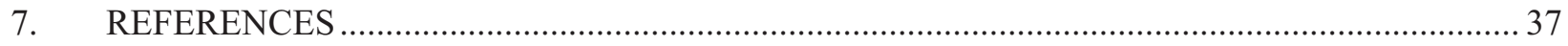

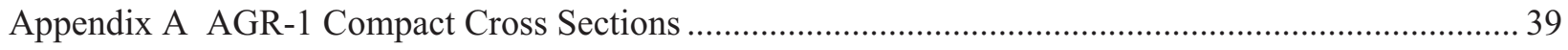

\section{FIGURES}

Figure 1. Photograph (left) and x-radiograph (right) of an unirradiated AGR-1 fuel compact. ..................2

Figure 2. Cutting diagram for AGR-1 compacts, illustrating how bright paint was used to preserve orientations of discrete sections for alignment with mount reference markers.

Figure 3. Periscope photos of as-cut transverse cross section of Compact 4-1-3 (a) and longitudinal cross sections of Compact 6-3-3 (b) after placing into Micarta mounts. 4

Figure 4. Cleanly cut particle at bottom end of a longitudinal cross section showing little internal damage.

Figure 5. Representative appearance of AGR-1 mounts near saw-cut surfaces after initial grinding and polishing (here transverse cross section of Compact 3-3-3) ........................................... 6

Figure 6. Shielded camera assembly before installation in the HFEF Containment Box.......................... 6 
Figure 7. Cropped images of the transverse cross section of Compact 2-1-3 taken by the new Containment Box camera: (a) 600-grit surface with most saw damage still present, (b) 600 -grit surface before last back-pot, and (c) after final 1- $\mu \mathrm{m}$ diamond polish.

Figure 8. Montage of transverse cross section of Compact 3-3-3 after incremental grinding and back-potting. (Compare to Figure 5 to see improved retention of kernels and buffers in Figure 8.)

Figure 9. Grinding damage before internal structures were stabilized by back-potting. ........................ 8

Figure 10. Continuous radial gap commonly seen in irradiated AGR-1 particle cross sections (a) and radial gap interrupted by residual buffer-IPyC bonding along the interface (b).

Figure 11. Characteristic end-state morphologies and associated features observed in irradiated AGR-1 fuel particles, where "i" signifies an intact buffer and "f" signifies a fractured buffer.

Figure 12. Particle morphology map for the transverse cross section of Compact 3-3-3.

Figure 14. Matrix fracture diverted around OPyC layer (a) and matrix-OPyC delamination (b), both from sample preparation.

Figure 18. Different buffer fracture shapes seen in Type Af and Type ABf particles.

Figure 19. Apparent incipient buffer fractures that initiated tangentially near azimuths where buffer-IPyC debonds terminated. 16

Figure 20. Tangential buffer fractures that propagated to varying extents. ........................................... 16

Figure 22. IPyC fractures in Type AB particles with multiple buffer fractures....................................... 18

Figure 24. Percentages of particles with IPyC fractures among irradiated AGR-1 compacts................... 19

Figure 25. Tangential SiC fractures bordering a probable soot inclusion................................................ 20

Figure 27. Pairs of SiC fractures symmetrically positioned around two buffer-IPyC fractures................21

Figure 29. Frequencies of IPyC fractures, IPyC-SiC debonds, and $\mathrm{SiC}$ fractures by type among the 830 classified AGR-1 particles. 22

Figure 30. Extensive kernel swelling at a plane between separated pieces of buffer. ….........................23

Figure 31. Kernels inhibited from swelling into buffer cavities by buffer fragments...............................2 24

Figure 32. IPyC interiors damaged by kernel growth through fractured buffer cavities. .........................24

Figure 33. Apparent radial gap between kernel and buffer in two Type B particles from Compact 6-3-3.

Figure 34. Larger kernel pores inside a fractured buffer than in a nearby kernel inside an intact buffer.

Figure 35 . Kernel pores within a fractured buffer that were only slightly larger than inside an intact buffer.

Figure 36. Larger pores in Type Bi Particles 061 and 023 than in nearby particles with fractured buffers

Figure 37. Two examples of spatial variations in pore enlargement.

Figure 38. Circles (white), polygons (red), and arcs (blue) fit to particles with (a) and without (b) a fractured buffer for dimensional measurements. 
Figure 39. Histogram of 95 irradiated cross-sectional kernel areas....................................................2 29

Figure 40. Kernel areas grouped according to buffer-imposed constraint on kernel growth..................... 30

Figure 41. Pore area percentages in kernels according to buffer-imposed constraint on kernel

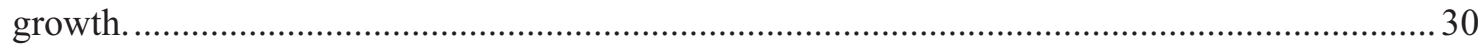

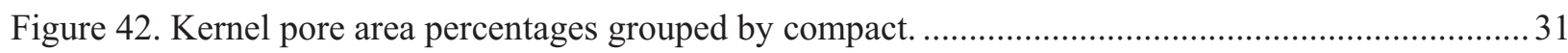

Figure 43. Size of largest pore in each kernel grouped by compact. ...................................................... 32

Figure 44. Largest kernel pore sizes grouped according to buffer-imposed constraint on kernel

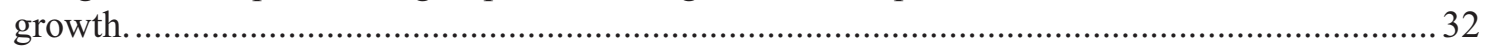

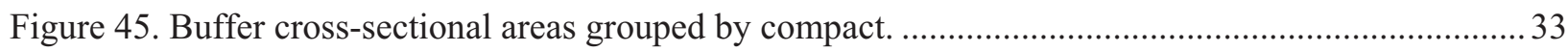

Figure 46. Buffer cross-sectional areas grouped according to buffer bonding and fracturing

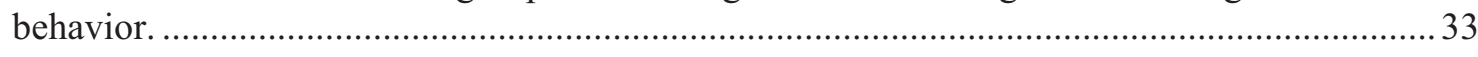

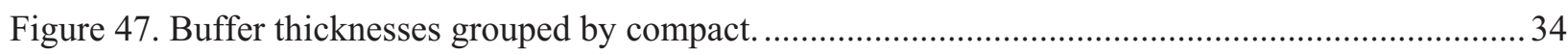

Figure 48. Buffer thicknesses grouped by buffer bonding and fracturing behavior. ............................... 34

Figure 49. Average buffer-IPyC gaps grouped by buffer densification and fracturing behavior. .............. 35

Figure 50. Average buffer-IPyC gaps grouped by compact. ............................................................... 35

Figure A-1. Transverse cross section through middle of Compact 2-1-3 (Mount 136T) with

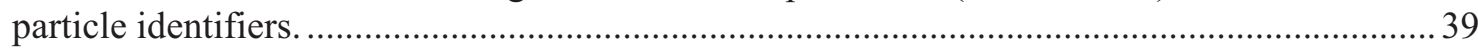

Figure A-2. Spatial distribution of particle morphologies across Compact 2-1-3 (Mount 136T).............. 40

Figure A-3. Longitudinal cross section through top portion of Compact 2-1-3 (Mount 137T) with

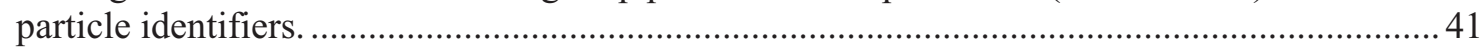

Figure A-4. Spatial distribution of particle morphologies across Compact 2-1-3 (Mount 137T).............. 42

Figure A-5. Longitudinal cross section through bottom part of Compact 2-1-3 (Mount 138T) with

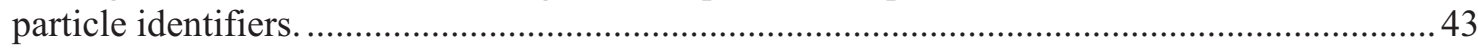

Figure A-6. Spatial distribution of particle morphologies across Compact 2-1-3 (Mount 138T).............. 44

Figure A-7. Transverse cross section through middle of Compact 3-3-3 (Mount 56T) with particle

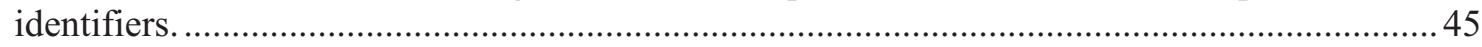

Figure A-8. Spatial distribution of particle morphologies across Compact 3-3-3 (Mount 56T)................ 46

Figure A-9. Longitudinal cross section through top portion of Compact 3-3-3 (Mount 62T).................. 47

Figure A-10. Longitudinal cross section through bottom part of Compact 3-3-3 (Mount 68T)................48

Figure A-11. Transverse cross section through middle of Compact 4-1-3 (Mount 59T) with

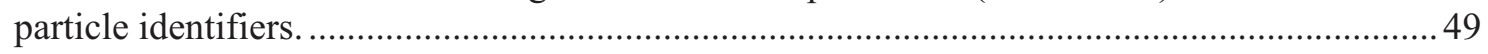

Figure A-12. Spatial distribution of particle morphologies across Compact 4-1-3 (Mount 59T).............. 50

Figure A-13. Longitudinal cross section through top portion of Compact 4-1-3 (in Mount 64T) with particle identifiers. The " $T$ " before the numbers denotes the top longitudinal section

Figure A-14. Spatial distribution of particle morphologies across top portion of Compact 4-1-3 (in Mount 64T). 
Figure A-15. Longitudinal cross section through bottom portion of Compact 4-1-3 (in Mount 64T) with particle identifiers. The " $\mathrm{B}$ " before the numbers denotes the bottom longitudinal section.

Figure A-16. Spatial distribution of particle morphologies across bottom portion of Compact 4-1-3 (in Mount 64T)...

Figure A-17. Transverse cross section through middle of Compact 5-1-2 (Mount 133T) with particle identifiers. .55

Figure A-18. Spatial distribution of particle morphologies across Compact 5-1-2 (Mount 133T)............ 56

Figure A-19. Longitudinal cross section through top portion of Compact 5-1-2 (Mount 134T) with particle identifiers.

Figure A-20. Spatial distribution of particle morphologies across top portion of Compact 5-1-2 (Mount 134T).

Figure A-21. Longitudinal cross section through bottom portion of Compact 5-1-2 (Mount 135T) with particle identifiers.

Figure A-22. Spatial distribution of particle morphologies across bottom portion of Compact 5-1-2 (Mount 135T)

Figure A-23. Transverse cross section through middle of Compact 6-3-3 (Mount 57T) with particle identifiers.

Figure A-24. Spatial distribution of particle morphologies across Compact 6-3-3 (Mount 57T).

Figure A-25. Longitudinal cross section through top portion of Compact 6-3-3 (in Mount 67T) with particle identifiers. The " $T$ " before the numbers denotes the top longitudinal section. This portion of the compact fractured during sawing.

Figure A-26. Spatial distribution of particle morphologies across top portion of Compact 6-3-3 (in Mount 67T).

Figure A-27. Longitudinal cross section through bottom portion of Compact 6-3-3 (in Mount 67T) with particle identifiers. The " $\mathrm{B}$ " before the numbers denotes the bottom longitudinal section.

Figure A-28. Spatial distribution of particle morphologies across bottom portion of Compact 6-3-3 (in Mount 67T).

\section{TABLES}

Table 1. Irradiation conditions for cross-sectioned AGR-1 compacts.................................................. 3

Table 2. Number of classified particles by type identified in each AGR-1 fuel compact.......................... 12

Table 3. Summary of IPyC fracture data from all classified particles in five AGR-1 fuel compacts.

Table 4. SiC anomalies in AGR-1 compacts, with unclassified particles in Figures 26 and 28(b) included. 


\section{ACRONYMS}

AGR Advanced Gas Reactor

ATR Advanced Test Reactor

FIMA fissions per initial metal atom

HFEF Hot Fuel Examination Facility

INL Idaho National Laboratory

IPyC inner pyrolytic carbon

MFC Materials and Fuels Complex

NDE nondestructive examination

OPyC outer pyrolytic carbon

ORNL Oak Ridge National Laboratory

PIE post-irradiation examination

TRISO tri-structural isotropic

VHTR very high temperature reactor 


\section{Ceramographic Examinations of Irradiated AGR-1 Fuel Compacts}

\section{INTRODUCTION}

\subsection{Background}

The Advanced Gas Reactor (AGR) Fuel Development and Qualification Program was established to perform the requisite research and development on tri-structural isotropic (TRISO)-coated particle fuel to support deployment of a very high temperature reactor (VHTR). The overarching goal of the program is to provide a baseline fuel qualification data set to support licensing and operation of a VHTR. To achieve these goals, the program includes the elements of fuel fabrication, irradiation, post-irradiation examination (PIE) and safety testing, fuel performance, and fission product transport [Simonds 2010].

Fuel irradiation experiments are being planned and conducted in the Advanced Test Reactor (ATR) at Idaho National Laboratory (INL). These experiments are intended to provide data on fuel performance under irradiation, support fuel process development, qualify the fuel for normal operating conditions, provide irradiated fuel for accident testing, and support the development of fuel performance and fission product transport models. The first of these irradiation tests, designated AGR-1, began in the ATR in December of 2006 and ended in November 2009. This experiment was primarily to act as a shakedown test of the multicapsule test train design and to provide early data on fuel performance that will be used in fuel fabrication process development. This test is also providing samples for post-irradiation safety testing, where fission product retention of the fuel at high temperatures will be experimentally measured. The capsule design and details of the irradiation experiment have been presented previously [Grover et al. 2010].

The AGR-1 fuel particles and compacts were fabricated at Oak Ridge National Laboratory (ORNL) using 350- $\mu \mathrm{m}$ diameter mixed uranium oxide/uranium carbide kernels produced by Backcock and Wilcox, Inc. The kernels were coated with a $100-\mu \mathrm{m}$ thick porous carbon buffer, a $40-\mu \mathrm{m}$ inner pyrolytic carbon (IPyC) layer, a 35- $\mu \mathrm{m}$ silicon carbide $(\mathrm{SiC})$ layer, and a $40-\mu \mathrm{m}$ outer pyrolytic carbon (OPyC) layer. The particles were pressed into right cylindrical compacts that were nominally $25 \mathrm{~mm}$ in length and $12.4 \mathrm{~mm}$ in diameter, which contained approximately 4,100 coated particles each (Figure 1). Uranium oxide/uranium carbide kernels are clearly visible in the x-radiograph shown in Figure 1, along with the $\sim 1.5-\mathrm{mm}$ thick fuel-free end caps at the top and bottom. A baseline fuel type and three fuel variants were included in the AGR-1 irradiation. All kernels came from a single composite lot with a uranium enrichment of $19.7 \%$ [Maki 2006]. Baseline fuel was fabricated using process conditions similar to historic German coating conditions, while Variant 1 had a higher IPyC deposition temperature, Variant 2 had a higher IPyC coating gas fraction, and Variant 3 had a lower SiC deposition temperature in conjunction with mixing an argon diluent into the hydrogen gas used to fluidize the particles [Maki 2006]. One key goal of the AGR-1 experiment is to identify any performance differences between the fuel types, either during irradiation or post-irradiation high temperature safety tests, in order to support optimization of the fuel fabrication process and eventual selection of a reference fuel for qualification.

A total of 72 compacts were irradiated in the AGR-1 experiment. The experiment completed 620 effective full power days of irradiation in the ATR. A peak burnup of $19.5 \%$ fissions per initial metal atom (FIMA) was achieved with zero particle failures observed based on the measured fission gas release-to-birth rate ratios [Grover 2010]. At completion of the irradiation, the test train was shipped to the Materials and Fuels Complex (MFC) at INL for PIE and high temperature safety testing. The primary objectives of the AGR-1 PIE and associated safety testing are to: (a) assess the overall performance of the 
test train and components and provide data to verify the test train thermal analyses, (b) evalute the fission product retention of the fuel during the irradiation and during high temperature post-irradiation safety tests, and (c) characterize the fuel compacts and individual particles to assess the condition of the matrix material, kernels, and coatings and document any concerns. Details of the activities planned as part of the PIE and safety testing, including the planned activities for specific compacts, have been described in the AGR-1 Post-Irradiation Examination Plan [Demkowicz 2010].
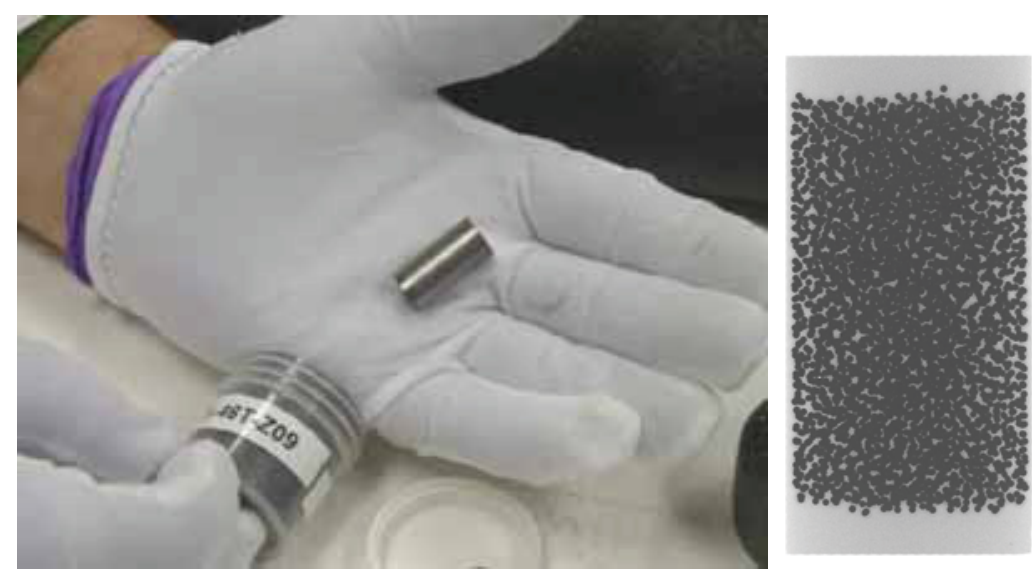

Figure 1. Photograph (left) and x-radiograph (right) of an unirradiated AGR-1 fuel compact.

Upon receipt at MFC, the AGR-1 test train was unpackaged, visually inspected, and underwent nondestructive examination (NDE) using gamma spectrometry. The test train was then sectioned into individual capsules and the capsules were opened to remove the fuel compacts and other internal capsule components for PIE. The fuel compacts, graphite fuel holders, and metal capsule shells were measured to determine the degree of dimensional change that occurred during the irradiation. Results of test train inspection and NDE, disassembly, and dimensional measurements have been reported in the AGR-1 Irradiated Test Train Preliminary Inspection and Disassembly First Look report [Demkowicz et al. 2011]. All of the irradiated AGR-1 fuel compacts were also gamma scanned in axial increments to determine the inventory of fission products and experimentally measure the burnup [Harp 2011]. Graphite fuel holders were also gamma scanned to detect and profile fission products released from the compacts to the adjacent holder material [Harp and Ploger 2011].

\subsection{Objectives for AGR-1 Compact Ceramography}

As outlined in the AGR-1 Post-Irradiation Examination Plan [Demkowicz 2010], microscopic examinations of cross-sectioned AGR-1 fuel compacts were primarily intended to investigate visually conspicuous phenomena such as cracking in the carbonaceous matrix, kernel deformation and porosity, buffer layer degradation, SiC layer corrosion, fractures in the TRISO layers and delaminations between them, and any deleterious interactions between the matrix and OPyC layer. Many of these objectives are also being pursued on epoxy-mounted groups of loose particles after deconsolidation. However, matrix behavior could only be studied in compact cross sections. Compact cross sections also provided the only opportunity to determine whether there were any spatial variations in particle behavior that might be related to temperature or burnup gradients within the compact. Spatial investigations necessarily required preserving microstructures and morphologies within as many particles as practicable against preparation damage. Consequently, an essential precursor to these activities was developing remote cutting, grinding, and polishing techniques that reliably produced high quality sample surfaces. Preserving the vast majority of particle features within these cross sections enabled characterizing large numbers of particles, which in turn enabled statistically significant determinations on particle behavior. 
As described in the AGR-1 Post-Irradiation Examination Plan [Demkowicz 2010], compacts were identified first by capsule number ( 1 through 6 ), then by elevation in each stack ( 1 at the bottom through 4 at the top), and finally by stack number $(1,2$, or 3$)$. A total of five irradiated AGR-1 compacts were examined at INL: Compacts 2-1-3, 3-3-3, 4-1-3, 5-1-2, and 6-3-3. Compact 6-4-2 is being examined at ORNL (results to be reported in a revision of this document). These compacts were chosen to span ranges of AGR-1 irradiation temperature [Hawkes 2012] and neutron exposure [Sterbentz 2011], as indicated in Table 1, and to represent speciments from each of the four fuel types. Additional ceramography is anticipated on a subset of the compacts subjected to high temperature accident tests.

Table 1. Irradiation conditions for cross-sectioned AGR-1 compacts.

\begin{tabular}{|c|c|c|c|c|}
\hline Compact & $\begin{array}{c}\text { Time-average volume- } \\
\text { average temperature }\left({ }^{\circ} \mathrm{C}\right)\end{array}$ & $\begin{array}{c}\text { Time-average maximum } \\
\text { temperature }\left({ }^{\circ} \mathrm{C}\right)\end{array}$ & $\begin{array}{c}\text { Average burnup } \\
(\% \text { FIMA })\end{array}$ & $\begin{array}{c}\text { Fast fluence } \\
\left(\mathrm{x} \mathrm{10} 0^{21} \mathrm{n} / \mathrm{cm}^{2}\right)\end{array}$ \\
\hline $2-1-3$ & 988 & 1097 & 18.4 & 3.77 \\
\hline $3-3-3$ & 1054 & 1147 & 19.0 & 4.30 \\
\hline $4-1-3$ & 1075 & 1184 & 19.3 & 4.20 \\
\hline $5-1-2$ & 1008 & 1115 & 15.7 & 3.38 \\
\hline $6-3-3$ & 1106 & 1174 & 13.5 & 2.70 \\
\hline $6-4-2$ & 1008 & 1100 & 11.2 & 2.17 \\
\hline
\end{tabular}

\section{SAMPLE PREPARATION}

All AGR-1 compacts were cut and marked in accordance with Figure 2, which created one transverse cross section and two longitudinal cross sections from each compact for examination. Cutting was conducted in the Containment Box at the MFC Hot Fuel Examination Facility (HFEF) with a Struers Minitom saw equipped with a Buehler $15 \mathrm{HC}, 127-\mathrm{mm}$ diameter diamond wafering blade. Saw speed was fixed at 200 revolutions per minute. Cutting force could be adjusted on the pivot arm holding the compact above the saw blade by sliding a counterweight with a master-slave manipulator. A nominal weight setting was established during test cuts on an unirradiated AGR-1 compact, but it was found necessary later to change the setting to compensate for blade wear (most of which occurred between batches of compacts on non-AGR samples) so that each cut took approximately 5 minutes. Preliminary cuts made dry and with water lubricant revealed no obvious advantage except wet cuts produced far less graphite dust. Several developmental modifications were made early in the sequence to fixtures that held full compacts for transverse cuts or end sections for longitudinal cuts. For example, before any irradiated AGR-1 compacts were cut, a second unirradiated compact was cross-sectioned for potential comparison to irradiated compacts. Inadequate thread length on the transverse fixture screws prevented firm clamping, so new screws had to be installed. A second longitudinal fixture with more travel also had to be built to accommodate end sections that were either longer or shorter than nominal.

Compact sections were gently loaded with manipulator-held tweezers into 32-mm diameter Micarta cylinders as shown in Figure 3. Each transverse section was loaded into a 19-mm diameter flat-bottomed recess. Both longitudinal sections initially were placed into an oval-shaped recess, but it proved difficult to keep both sections at the same height as well as parallel to the Micarta surface. Later longitudinal sections were individually loaded into their own Micarta mounts. The shiny holes in Figure 3 correspond to matrix holes where particles were pulled out by the saw and to empty IPyC layers from missing buffers and kernels. At least as many particles were damaged (buffers/kernels lost or entire particles dislodged) by the saw as cut cleanly by the saw. Some section corners and edges also broke off during saw cuts, as can be seen where the sections butt together in Figure 3(b). Despite these gross forms of saw damage, sawing did minimal damage to internal particle structures when particles were cut cleanly, as 
demonstrated by the bottom surface in Figure 4. Note that Particle AGR1-213-137T-058 in Figure 4 was denoted by experiment (AGR-1), by compact (2-1-3), by mount number (137T), and by order of imaging (058). All examined particles were individually identified using this format.
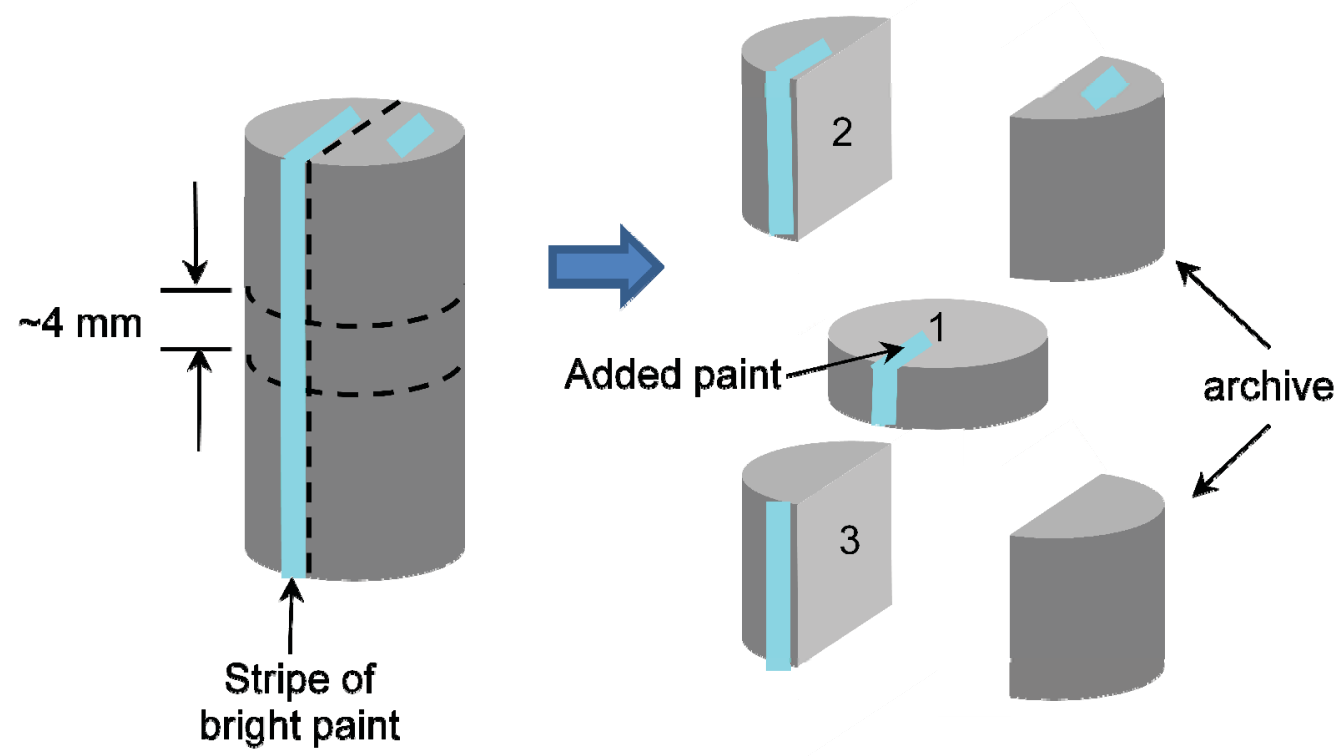

Figure 2. Cutting diagram for AGR-1 compacts, illustrating how bright paint was used to preserve orientations of discrete sections for alignment with mount reference markers.
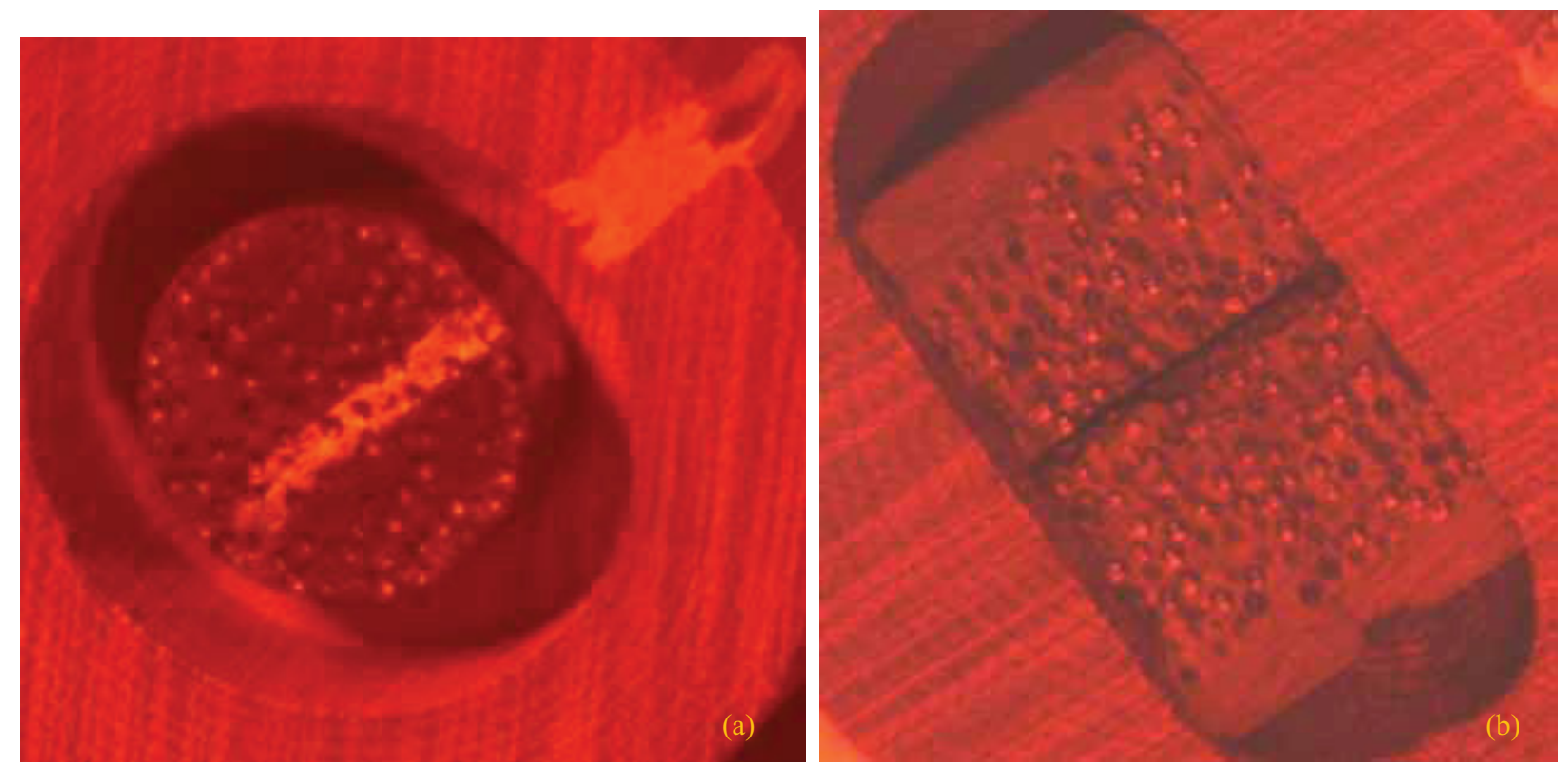

Figure 3. Periscope photos of as-cut transverse cross section of Compact 4-1-3 (a) and longitudinal cross sections of Compact 6-3-3 (b) after placing into Micarta mounts. 


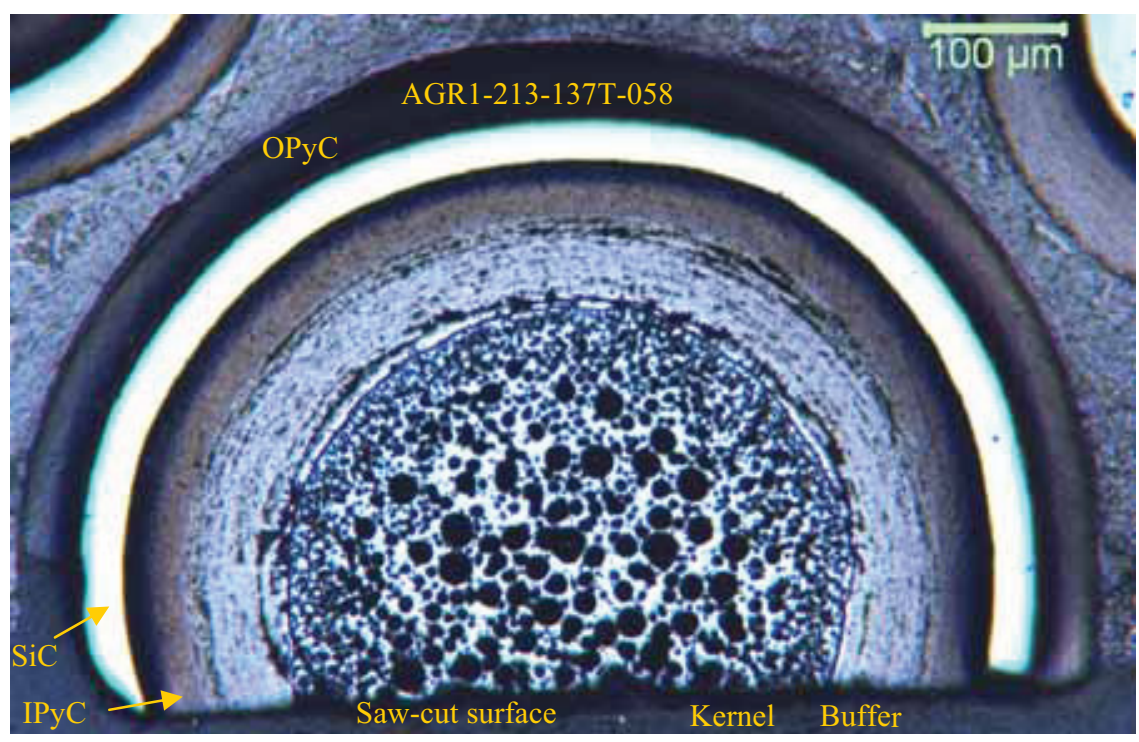

Figure 4. Cleanly cut particle at bottom end of a longitudinal cross section showing little internal damage.

After pouring in Buehler EpoHeat two-part epoxy, each mount was placed under a bell jar and a vacuum was applied to remove bubbles of trapped gas. This process was repeated twice. The epoxy was cured using heat from hot cell lamps for approximately 2 hours. Mounts were roughly ground with a Struers MD-Piano 80-grit resin-bonded diamond disc under 40 Newtons $(\mathrm{N})$ of force until the epoxy overlayer was removed. Compact surfaces were then back-potted by dribbling Buehler EpoHeat epoxy onto the mounts with a manipulator-held eyedropper, applying a vacuum for three cycles, and curing under hot cell lamps. Grinding then proceeded with a Struers MD-Piano 600-grit disc under $20 \mathrm{~N}$ of force until all surplus back-potted epoxy was removed. Because the back-potted epoxy was not applied evenly, some compact was also removed before all back-potted epoxy was gone. Final polishing was then performed using a Struers MD-Piano 1200-grit disc, 3- $\mu \mathrm{m}$ diamond polish with a MD-Mol cloth, and $1-\mu \mathrm{m}$ diamond polish with a MD-Nap cloth-all for 5-minute durations under $20 \mathrm{~N}$ of force.

After pneumatically transferring the mounts to the Leitz metallograph, black and white images were taken with a 6.6-megapixel Leaf Volare camera. Results were disappointing as demonstrated by the Figure 5 montage (wavy bands are a stitching artifact from uneven illumination). Most kernels and buffers were missing (empty shells appear black in Figure 5 while those filled with back-potted epoxy appear gray). The darker spot near the bottom of Figure 5 is an empty matrix hole where a particle pulled out during grinding. Virtually all of the original saw damage remained, and additional kernels and buffers were lost during the grinding and polishing process. (Most of these kernels and buffers lacked lateral support after their buffers densified during irradiation, especially in particles ground past midplane.) With so few particles retaining their kernels and buffers, prospects for finding any spatial variations in particle morphologies appeared poor. Furthermore, the possibility existed that some morphologies might have been lost preferentially, such as particles with fractured buffers. Consequently, a technique had to be developed to grind past the saw damage without losing many additional kernels and buffers in newly exposed particles. The strategy was to gradually grind with a 600 -grit disc with incremental back-potting of epoxy and vacuum impregnation to stabilize emerging kernels and buffers. Because a mount thickness of at least one-half a particle diameter had to be ground off to get beyond empty holes and shells missing kernels and buffers, a minimum of three back-pots had to be performed while saw damage was removed.

Determining when the next back-pot was needed relied upon observing the progression of IPyC layer break-through, which opened access into gaps between densified buffers and IPyC layers. Periscope examinations were augmented with an inexpensive digital camera apparatus, which both provided more 
detailed views and also enabled before-and-after comparisons. As displayed in Figure 6, the Hitachi KP-M2AN camera was surrounded with surplus pieces of tungsten alloy shielding, while browning of the Computar TEC-55 lens by the mounts was mitigated by a remotely tilted mirror. In actual hot cell use, mounts were illuminated by boom-held lamps. The average shielding thickness of $38 \mathrm{~mm}$ allowed a camera service life of nearly 3 months, despite the high local gamma field from stored samples of irradiated fuel.

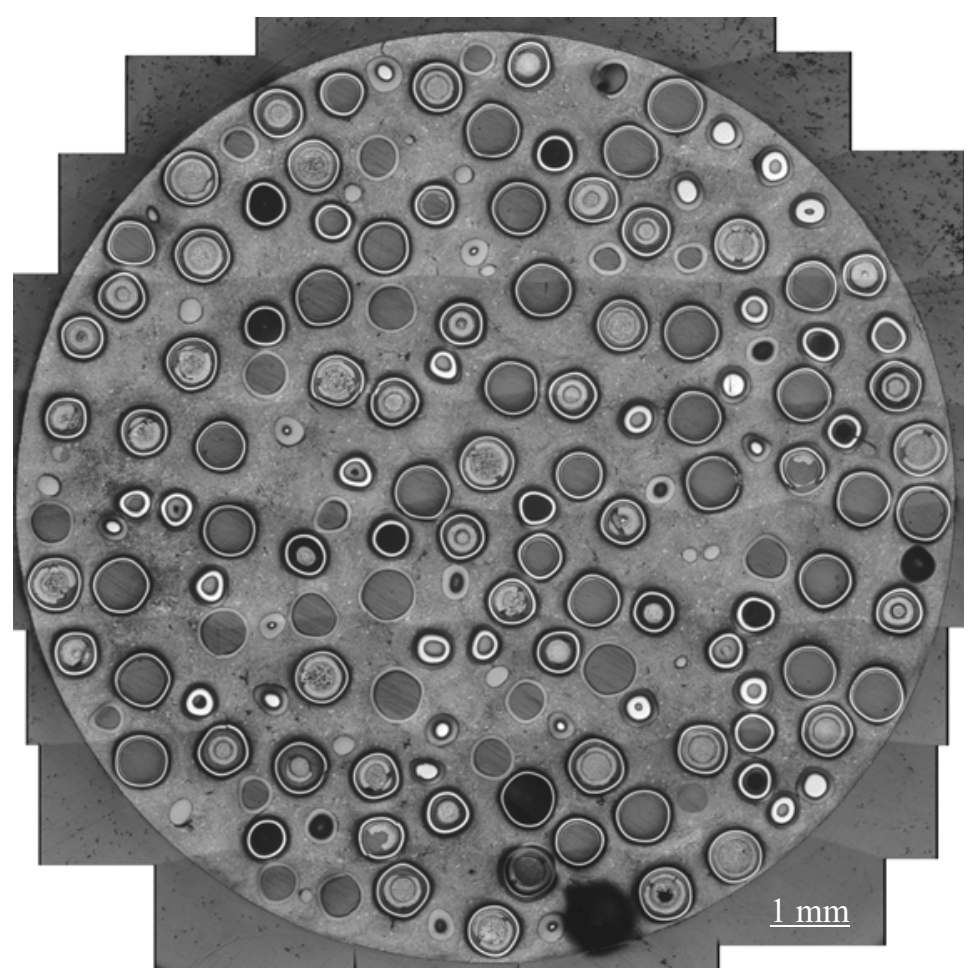

Figure 5. Representative appearance of AGR-1 mounts near saw-cut surfaces after initial grinding and polishing (here transverse cross section of Compact 3-3-3).

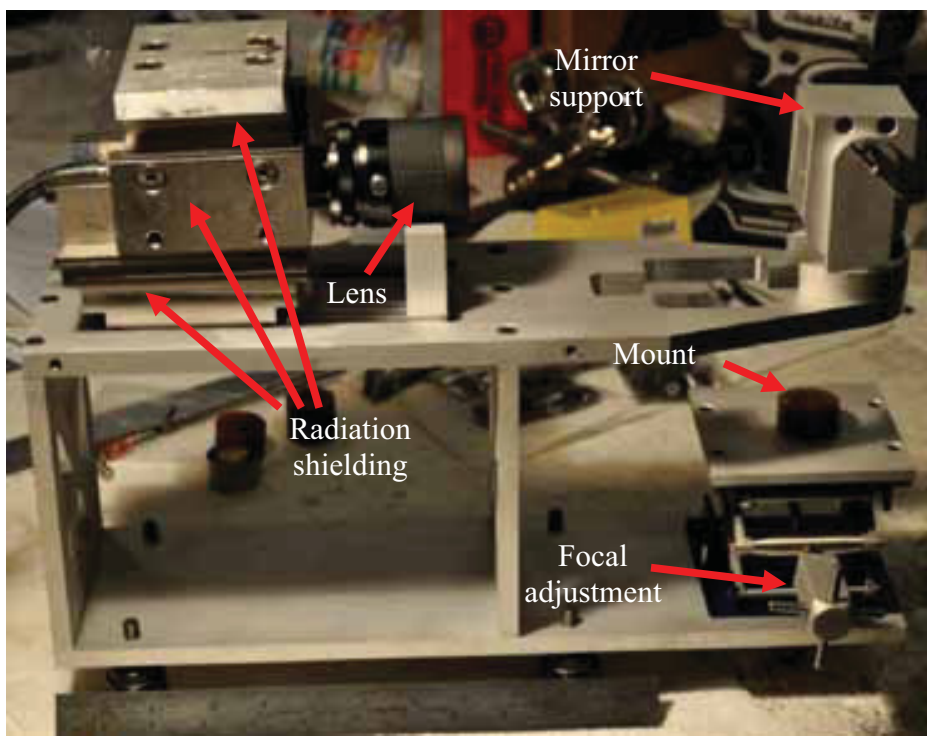

Figure 6. Shielded camera assembly before installation in the HFEF Containment Box. 
The progression in mount appearance over the incremental grinding and back-potting process is illustrated in Figure 7. The Containment Box camera resolution was constrained to 0.4 megapixel by the available cable feedthrough. Nevertheless, the resolution was adequate to distinguish features of interest, especially the dark areas in Figure 7(b) that indicated open buffer-IPyC gaps in need of back-potting before more grinding. Good retention of kernels and buffers is even more evident in the metallograph montage in Figure 8. A goal of $75 \%$ retention (losing no more than approximately 15 kernels/buffers per mount) had been established for meaningful spatial investigations, which was satisfied by all of the reground AGR-1 cross sections. However, Figure 8 demonstrates that nearly all kernels and buffers could be retained with ample time and care.
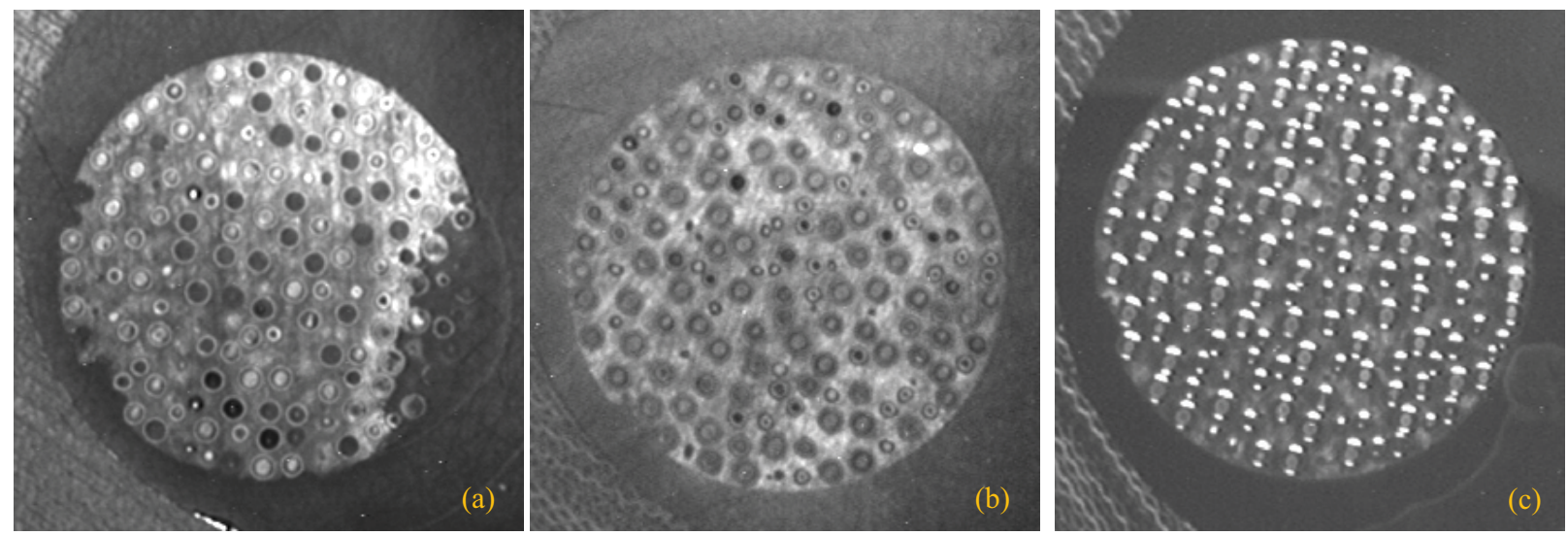

Figure 7. Cropped images of the transverse cross section of Compact 2-1-3 taken by the new Containment Box camera: (a) 600-grit surface with most saw damage still present, (b) 600-grit surface before last backpot, and (c) after final 1- $\mu \mathrm{m}$ diamond polish.

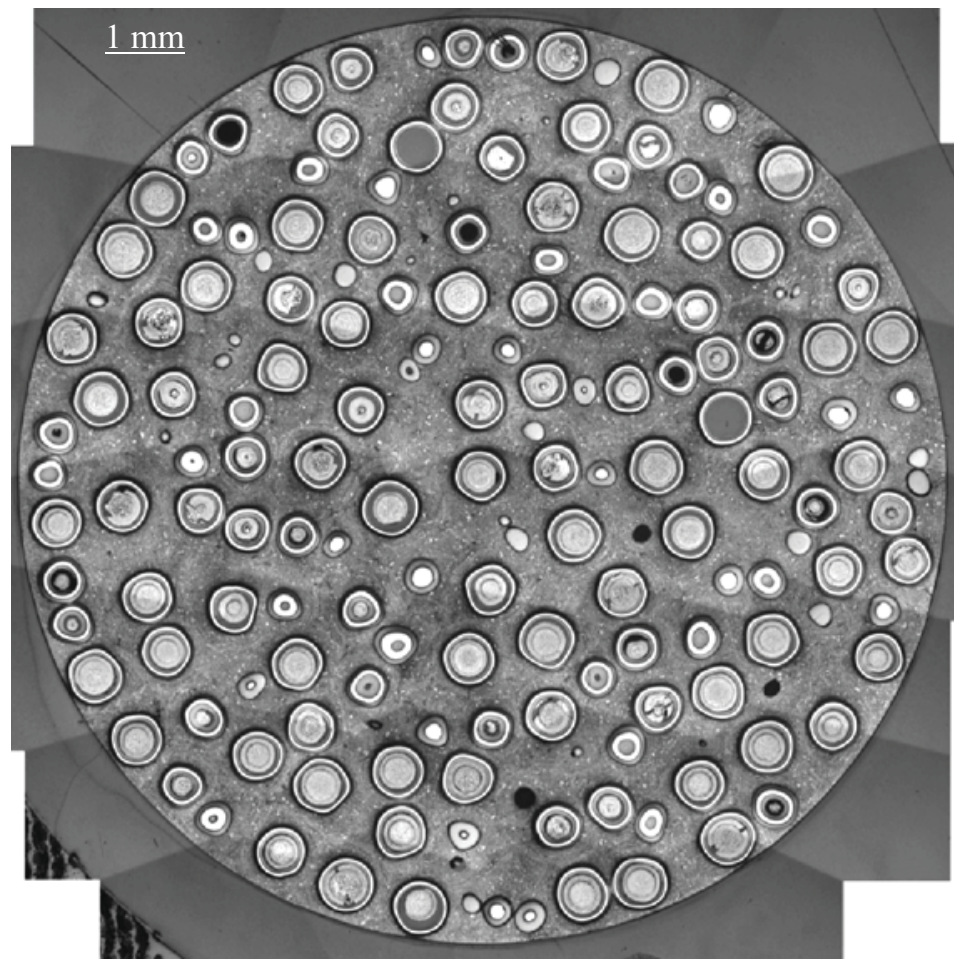

Figure 8. Montage of transverse cross section of Compact 3-3-3 after incremental grinding and back-potting. (Compare to Figure 5 to see improved retention of kernels and buffers in Figure 8.) 
Incremental grinding and back-potting were more effective when the new ground surface was parallel to the surface before back-potting so that an equal amount of material was removed across the entire cross-sectional surface. This required laying down an even layer of epoxy each time, but epoxy was uneven after applying with an eyedropper. Foam-tipped swabs were found to be nearly ideal for smoothing out the back-potted epoxy across the compact cross sections. At first, the smoothing was done after vacuum impregnation, but further optimization reduced the amount of epoxy that had to be applied. With practice, a single drop of epoxy was adequate for complete coverage before vacuum impregnation, which also minimized the grinding time necessary for removing surplus back-potted epoxy.

Despite the dramatic improvement in retention of kernels and buffers from frequent back-potting, isolated particles with minor amounts of grinding damage could still be found. Figure 9 provides one example where the buffer and kernel rotated several times inside the IPyC layer while being ground before being stabilized by back-potting. A lenticular IPyC fracture apparently also occurred where the buffer rotated against the IPyC layer, and hairline fractures may have occurred in the buffer at the same time. Although most buffer-IPyC gaps were impregnated with epoxy before the buffer was contacted by the grinding disc, one or two such lopsided buffers were typically found in each of the compact cross sections. IPyC damage from buffer rotation was found to be extremely rare during sample preparation.

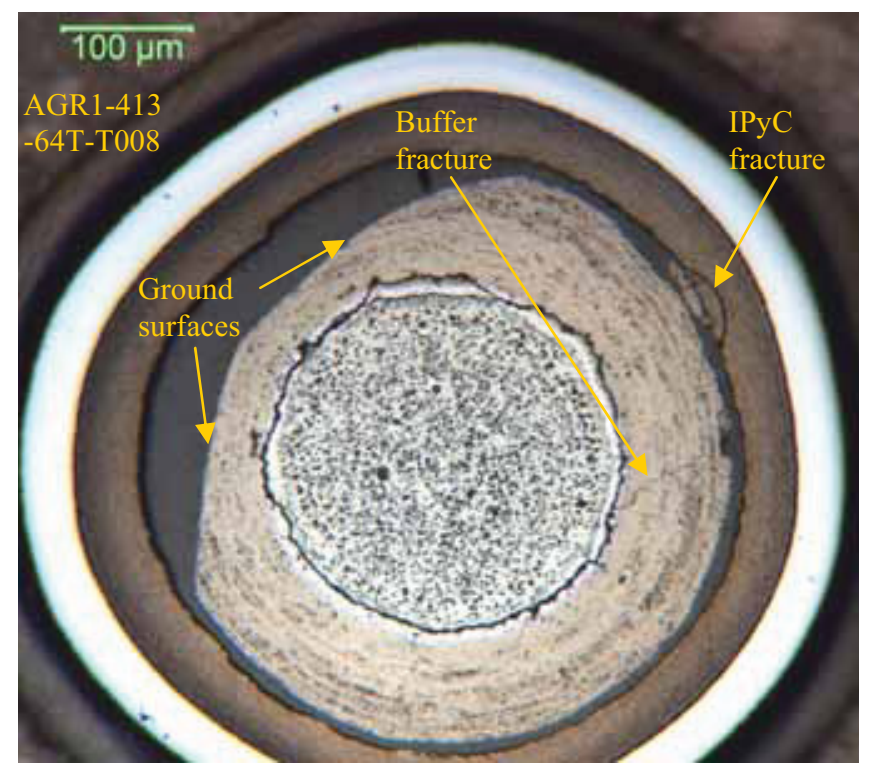

Figure 9. Grinding damage before internal structures were stabilized by back-potting.

\section{PARTICLE INSPECTION RESULTS}

The general microscopy procedure used on the Leitz metallograph with a newly installed Jenoptik ProgRes C14+ variable resolution camera was as follows:

1. Cover each mount with low magnification, 1.4-megapixel, black and white images and then assemble them into a montage using Adobe Photoshop (see example in Figure 8).

2. Take intermediate magnification, 5.6-megapixel, white-balanced color images of each particle ground near enough to midplane to expose some kernel. Particle identifiers were recorded in sequence on each montage. (Montages of all AGR-1 compact cross sections are presented with particle identifiers in Appendix A.) Consistent contrast, brightness, and exposure settings were used to facilitate measurement comparisons.

3. Take high magnification, high resolution, color images of features of particular interest. 
The approach of taking high resolution, intermediate magnification images only on particles with visible kernels meant that some features of key interest such as $\mathrm{SiC}$ fractures could have been missed in particles ground too far from midplane to expose kernel. Consequently, the montages were carefully scanned later for candidate particles with potential $\mathrm{SiC}$ fractures; three mounts had to be reexamined. In two of these mounts, one particle with no exposed kernel was found to contain at least one $\mathrm{SiC}$ fracture, so additional high magnification images were taken of these two particles.

\subsection{Characteristic Morphologies}

Several characteristic morphologies came to be recognized as the high resolution images of individual particle cross sections were examined. The most common feature was a radial gap between the buffer and IPyC layers where the buffer densified inward (outer surface moved toward kernel) during irradiation, as displayed in Figure 10(a). In some particles with inward buffer densification, such as the one shown in Figure 10(b), the radial buffer-IPyC gap was interrupted by regions where the buffer stayed firmly bonded to the IPyC layer. Many of these particles debonded along most of the interface, while debonding was much less extensive on some other particles when the AGR-1 irradiation ended. The rarest case was where a particle had no buffer-IPyC gap. In these cases, the buffer stayed bonded to the IPyC layer around the entire circumference. The buffers in these particles still evidently densified, but the inner buffer surface moved radially outward rather than the outer buffer surface moving inward. Kernels in these particles often had larger pores than seen inside kernels within inwardly densified buffers.
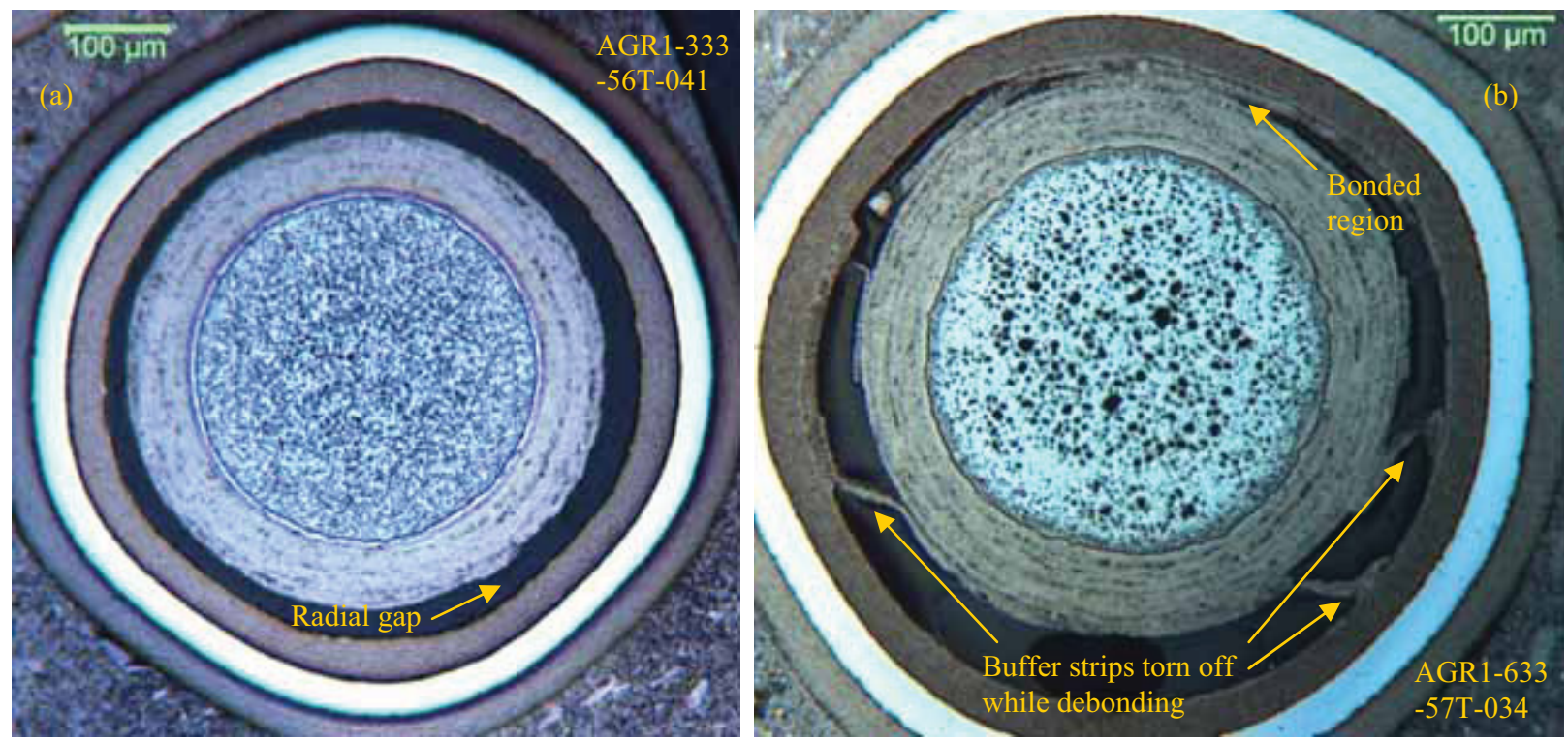

Figure 10. Continuous radial gap commonly seen in irradiated AGR-1 particle cross sections (a) and radial gap interrupted by residual buffer-IPyC bonding along the interface (b).

A comprehensive scheme for classifying irradiated AGR-1 particles by morphology in the plane of polish is presented with representative examples in Figure 11. A radial buffer-IPyC gap around the entire circumference (completely detached buffer-IPyC interface) is designated as a Type A end-state morphology. Particles with fully bonded buffer and IPyC layers are termed Type B particles. Debonding along only part of the buffer-IPyC interface is called a Type AB morphology. Buffer fracturing occurred in Types $\mathrm{A}, \mathrm{B}$, and $\mathrm{AB}$, where buffer fractures were often accompanied by kernel pore enlargement and kernel growth between buffer pieces. Because other consequences of buffer fracturing could be quite different in the three basic types (as discussed below), six characteristic morphologies were used to classify irradiated AGR-1 particles, as displayed in Figure 11. 


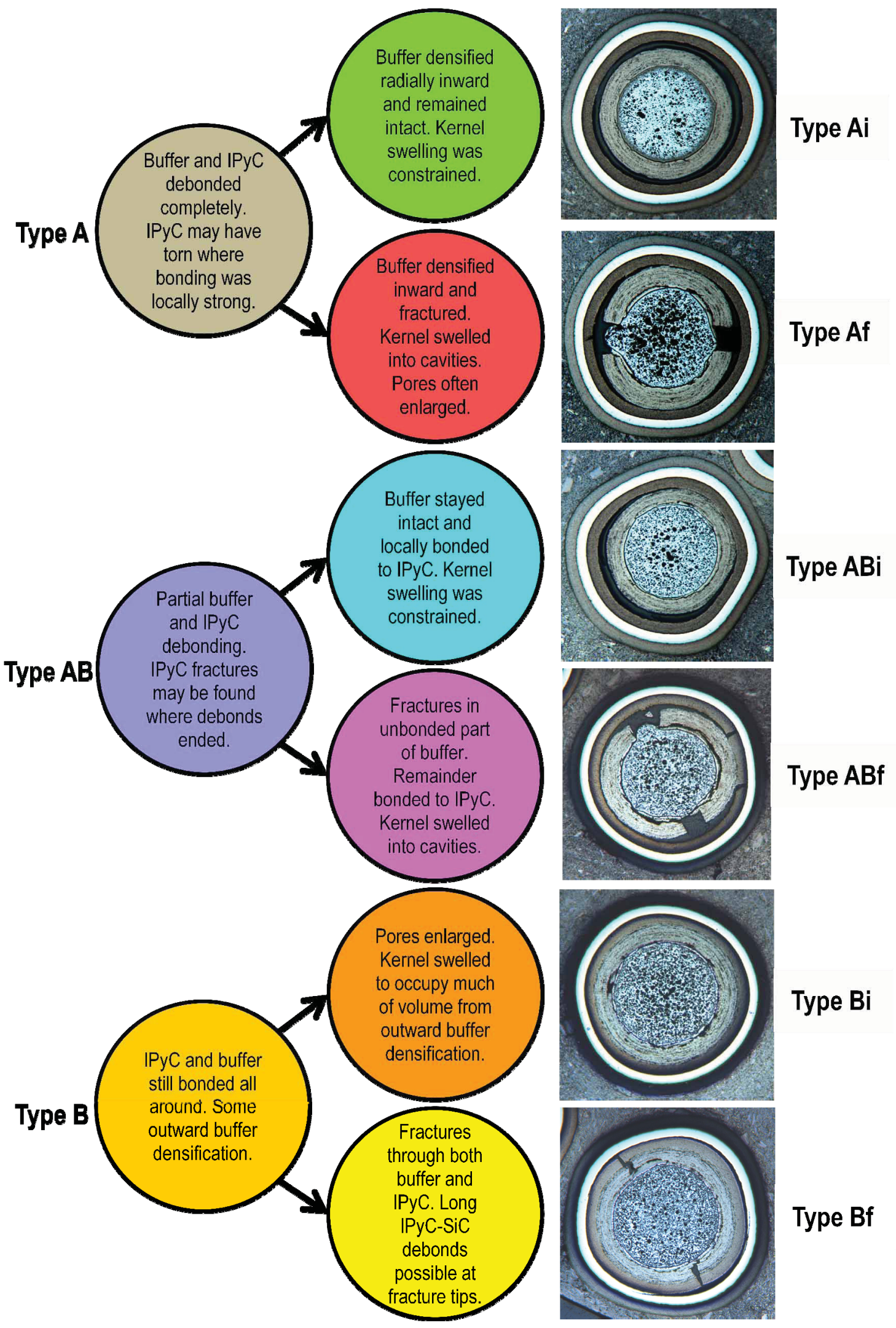

Figure 11. Characteristic end-state morphologies and associated features observed in irradiated AGR-1 fuel particles, where "i" signifies an intact buffer and "f" signifies a fractured buffer. 
Firmly distinguishing between Types $\mathrm{A}$ and $\mathrm{AB}$ was difficult with only a two-dimensional (2-D) view. In Type A particles, the possibility of local buffer-IPyC bonding cannot be eliminated for planes not visible in the micrograph. Furthermore, as illustrated by the Type AB particle in Figure 10(b), buffers did not always detach smoothly from IPyC layers, especially where bonding was locally strong. Patches of buffer occasionally remained attached to IPyC interiors in Type A particles.

The colors in the middle column of Figure 11 were used to encircle the classified particles in the cross sections in order to map morphologies. An example map is provided in Figure 12, where the spatial distribution of colors appears random. This is typical of all AGR-1 transverse and longitudinal morphology maps presented in Appendix A. No obvious patterns emerged despite appreciable estimated axial and radial gradients in temperature [Hawkes 2012], some axial variation in calculated fast fluence [Sterbentz 2011], and significant axial variations in measured burnup [Harp 2011] within individual irradiated AGR-1 compacts.

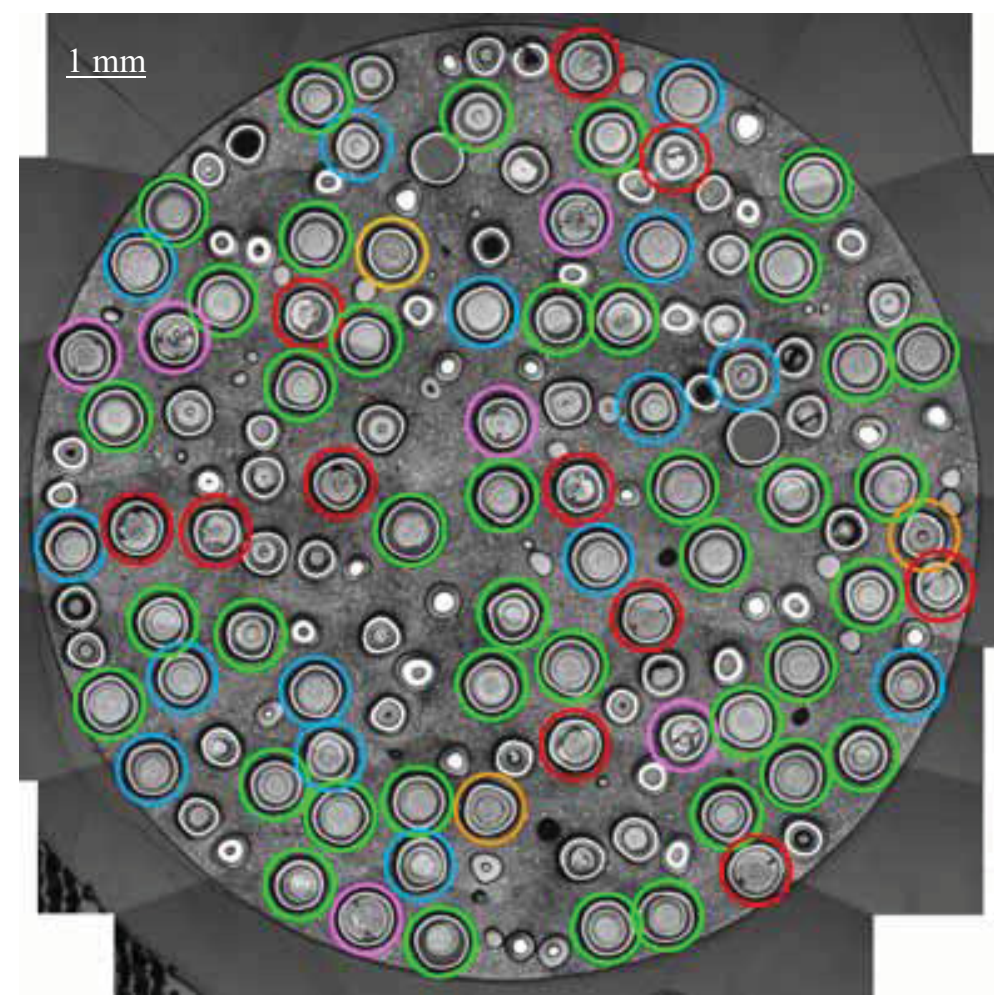

Figure 12. Particle morphology map for the transverse cross section of Compact 3-3-3.

\subsection{Particle Type Frequencies}

Frequencies of morphological types varied among compacts, as shown in Table 2. The most evident observation concerns Compact 6-3-3, where somewhat more buffer fracturing was found (Type Af plus Type ABf) at the expense of Type Ai particles, relative to the other four compacts. Note that numbers of particles with fractured buffers in Table 2 were biased upward slightly because of how particles were selected for classification. High resolution images were only taken of particles with a visible kernel, but this selection method included several Type Af and Type ABf cases where small kernel portions intruded into buffer cavities perpendicular to the micrograph plane. Without such kernel intrusions into perpendicular buffer cavities, these particles would not otherwise have been classified because they were ground too far from midplane to expose the main kernel portion. Also note in Table 2 that relatively few particles were classified from Compact 3-3-3 because only the central transverse cross 
section from this compact was incrementally ground and back-potted beyond saw damage. All three mounts were examined from the other four compacts.

Table 2. Number of classified particles by type identified in each AGR-1 fuel compact.

\begin{tabular}{|c|c|c|c|c|c|c|c|}
\hline \multirow{2}{*}{ Compact } & \multicolumn{2}{|c|}{ Type A } & \multicolumn{2}{c|}{ Type B } & \multicolumn{2}{c|}{ Type AB } & \multirow{2}{*}{ Totals } \\
\cline { 2 - 7 } & $\mathrm{Ai}$ & $\mathrm{Af}$ & $\mathrm{Bi}$ & $\mathrm{Bf}$ & $\mathrm{ABi}$ & $\mathrm{ABf}$ & 206 \\
\hline $2-1-3$ & 107 & 15 & 7 & 2 & 44 & 31 & 75 \\
\hline $3-3-3$ & 38 & 9 & 3 & 0 & 17 & 8 & 157 \\
\hline $4-1-3$ & 95 & 8 & 10 & 0 & 31 & 13 & 205 \\
\hline $5-1-2$ & 123 & 14 & 3 & 2 & 39 & 24 & 187 \\
\hline $6-3-3$ & 72 & 31 & 17 & 2 & 33 & 32 & \multirow{2}{*}{830} \\
\hline \multirow{2}{*}{ Totals } & 435 & 77 & 40 & 6 & 164 & 108 & 272 \\
\hline
\end{tabular}

Data from Table 2 were reconfigured according to the three basic particle types and then normalized into percentages for presentation in Figure 13. Buffer-IPyC debonding clearly was very common in irradiated AGR-1 particles. Fully intact buffer-IPyC bonds (Type B) were found in less than $6 \%$ of the 830 classified particles, while complete buffer-IPyC debonding (Type A) was seen in nearly $62 \%$ of the particles. Particles with partial debonding constituted approximately $33 \%$ of the population. Most images of Type ABi particles revealed that debonding occurred along much of their buffer-IPyC boundaries, so it appears that many Type ABi particles were transforming to Type Ai when the AGR-1 experiment was concluded. Figure 13 suggests that differences in neutron exposure and temperature among AGR-1 compacts (see Table 1) had only minor influence on the extent of buffer-IPyC debonding. Variations in AGR-1 TRISO coatings also had no obvious impact on how much debonding occurred along interfaces between buffer and IPyC layers.

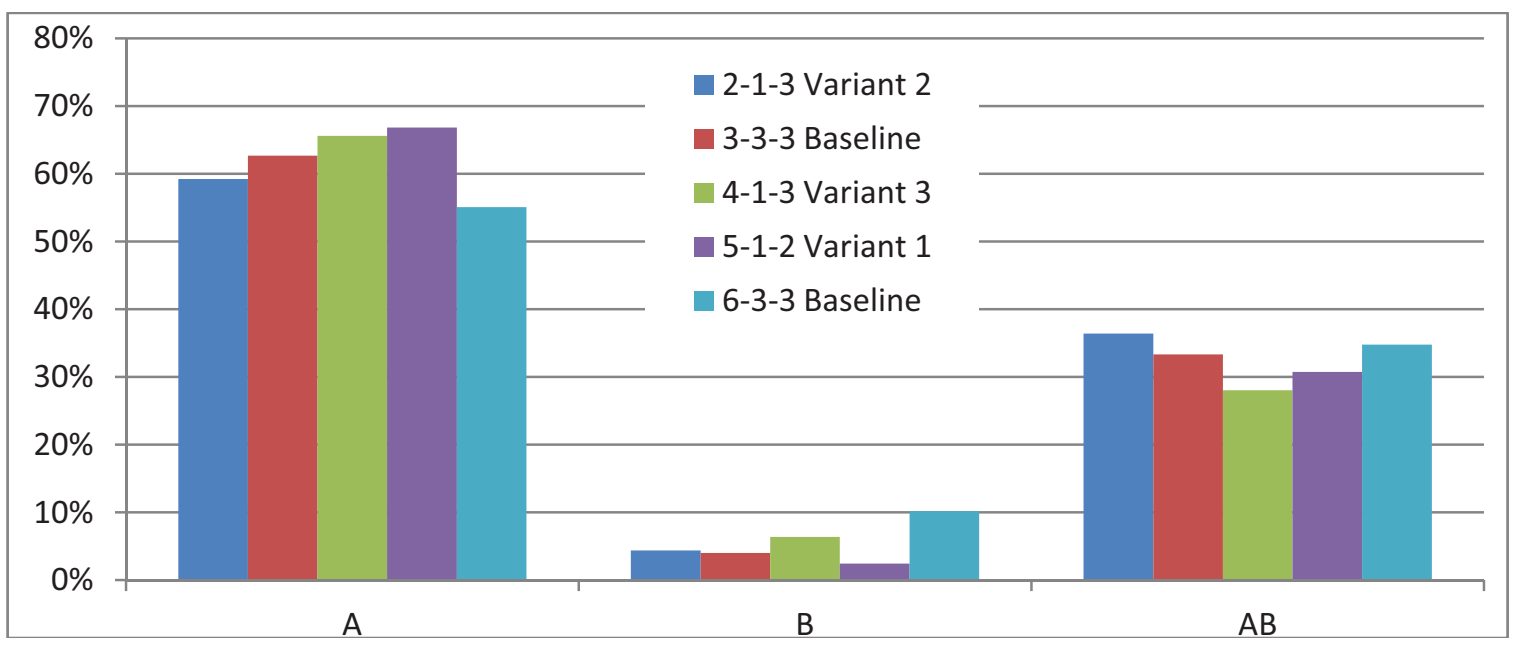

Figure 13: Percentages by AGR-1 compact within each of the three basic particle types.

It is likely that the range of debonding behavior at the buffer-IPyC boundary is related to variation in the strength of that interface. The AGR-1 buffers were deposited at a much greater rate (five to six times faster) than the pyrocarbon layers. This produced the desired porosity but also resulted in the greatest variation in microstructure. The strength of the buffer-IPyC interface can be affected by the porosity at the buffer surface. Open porosity encourages IPyC infiltration that produces a stronger interface. 


\subsection{TRISO Layer Fractures}

Zero in-pile particle failures were detected from abrupt fission gas releases during the AGR-1 experiment. These abrupt fission gas releases would be expected to occur if the three gas-tight layers (IPyC, $\mathrm{SiC}$, and $\mathrm{OPyC}$ ) all failed. However, irradiation-induced fractures of varying severity and frequency were found during ceramography in all TRISO layers except the OPyC layer. No OPyC fractures were observed. Even on the occasions when the carbonaceous compact matrix fractured during sample preparation, the fractures always diverted around the OPyC layers rather than propagating into them. Similarly, the only instances of matrix-OPyC debonding have been attributed to sample preparation. Examples of both phenomena are provided in Figure 14. Both particles shown in Figure 14 are from the same longitudinal cross section that fractured during sawing because of inadequate clamping. (The " $T$ " before the particle numbers in Figure 14 signifies the top longitudinal section, as discussed in Appendix A, because both longitudinal sections from Compact 6-3-3 were loaded into Mount 67T.)

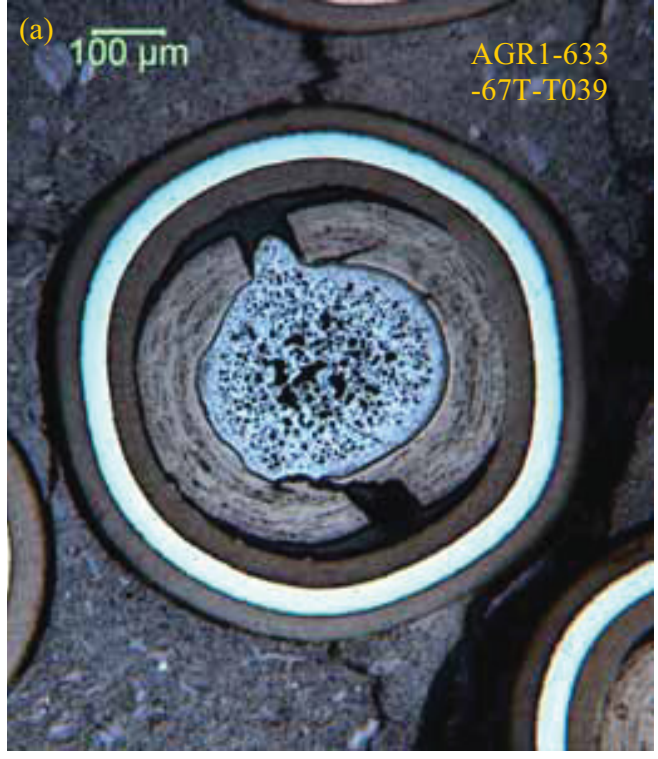

Figure 14. Matrix fracture diverted around $\mathrm{OPyC}$ layer (a) and matrix-OPyC delamination (b), both from sample preparation.

\subsubsection{Buffer Fractures}

Fractures were far more frequent in the irradiated AGR-1 buffers than in any other TRISO layer. As displayed in Figure 15 (data combined from Table 2), buffer fractures were seen on average in $23 \%$ of the 830 classified particles. However, as noted previously, the selection method biased Type Af and Type ABf examinations upward slightly so all percentages in Figure 15 should be viewed relatively rather than in a strict absolute sense.

Figure 15 shows that Type $\mathrm{A}$ and Type $\mathrm{AB}$ buffer fractures were somewhat more frequent than average in Compact 6-3-3 and less common in Compact 4-1-3. Overall, however, the minor buffer fracture frequency differences among compacts were less striking than the frequency differences among particle types. Although Type Bf particles were too rare for meaningful comparisons, Figure 15 reveals that buffer fractures occurred over twice as frequently in Type AB particles than in Type A particles. This general trend was seen for all five compacts. The reason was not immediately apparent because buffer fractures have been thought to arise in both Type A and Type AB particles from buffer hoop stress created by the combination of inward buffer densification and outward kernel growth. The diametrically 
opposed buffer fractures shown in the Figure 11 examples for Type Af and Type ABf particles likely developed as illustrated in Figure 16, where one side fractured earlier and allowed preferential kernel growth into the initial cavity. Most of the Type Af and Type ABf particles showed more kernel growth into a cavity on one side. Fractured buffer surfaces from the Figure 16 process typically were purely in the radial direction with smooth contours.

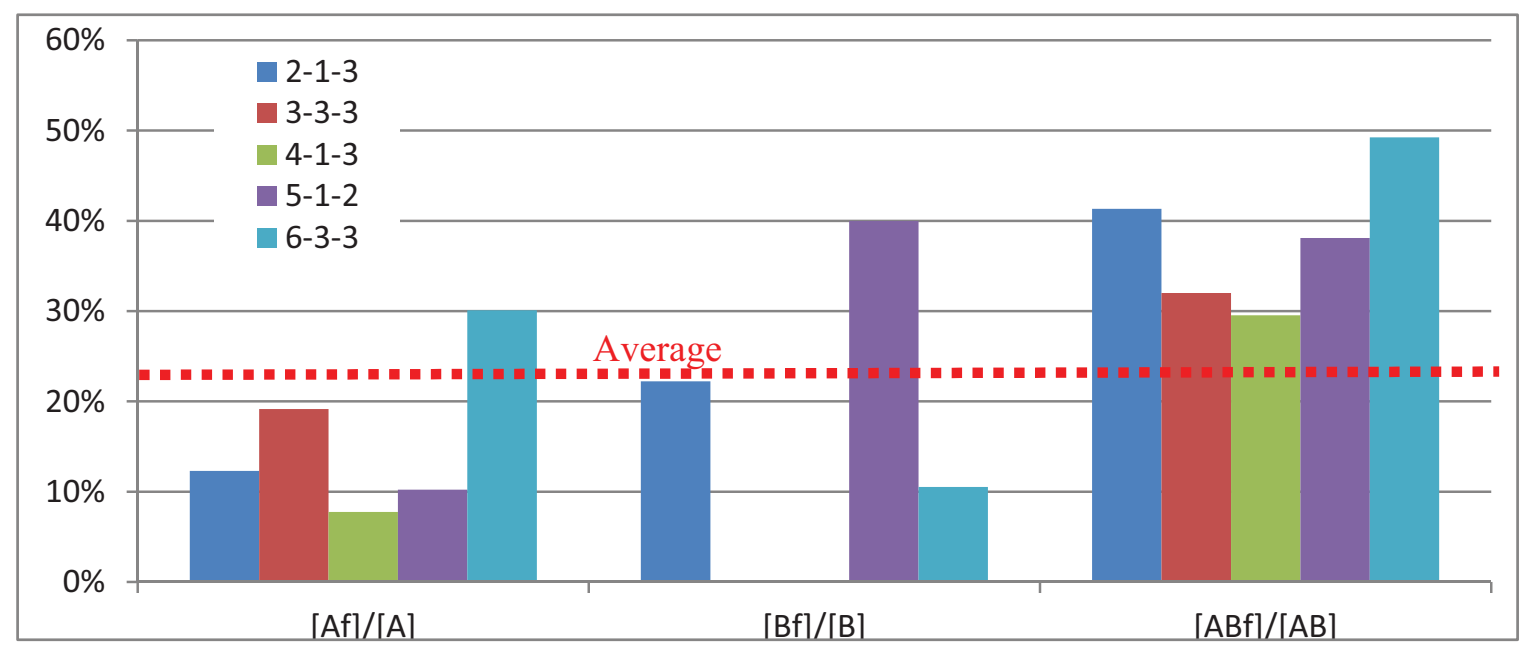

Figure 15. Frequency of fractured buffers among basic types in irradiated AGR-1 compacts.

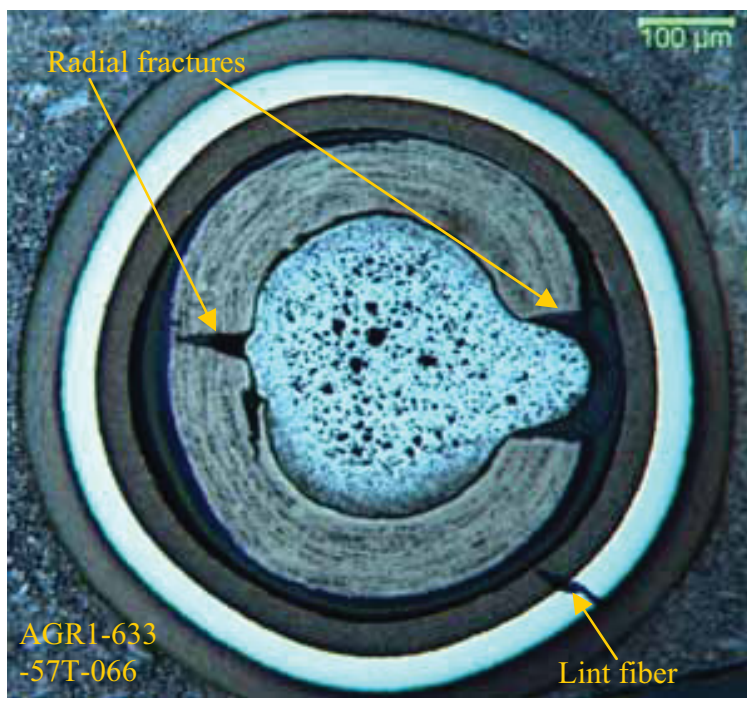

Figure 16. Preferential kernel growth into a cavity from the initial buffer fracture.

The buffer fracture process in Figure 16 cannot explain the preponderance of buffer fractures in Type AB particles, where a second mechanism may have dominated. Figure 17 shows that buffer fractures could be more complex than in Figure 16, where the complex fractures in Figure 17 often appear to have initiated tangentially at the kernel interface. The initially tangential buffer fractures evidently failed in the radial direction after propagating in a variety of directions, leaving fracture surfaces with a jagged contour. Figure 14(a) shows a smooth radial fracture on the top side and a jagged contour on the bottom, so some particles exhibited both shapes of buffer fracture. 

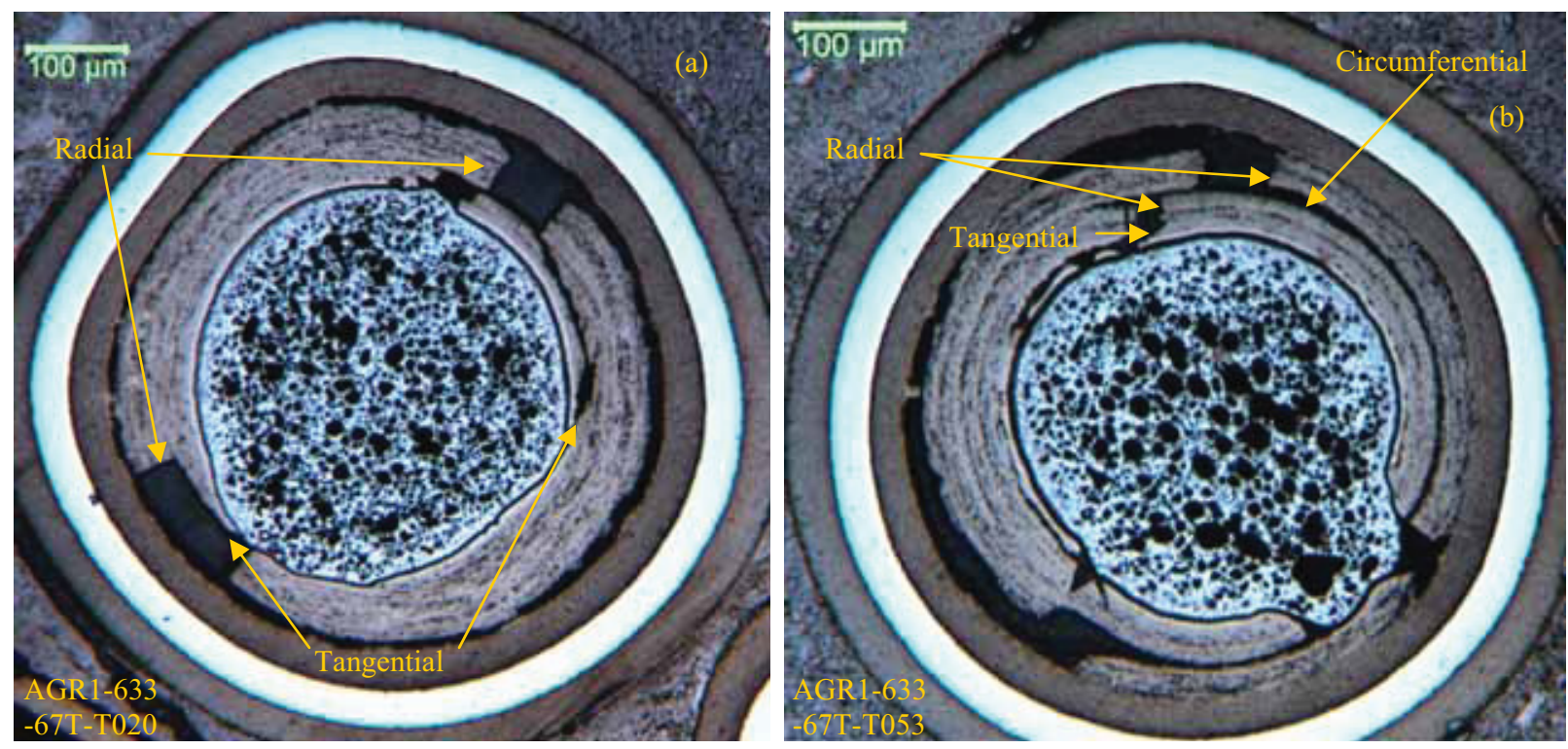

Figure 17. Jagged buffer fractures with mixtures of tangential, radial, and occasionally circumferential components.

Figure 18 reveals that smooth radial fractures were much more common in Type Af buffers than in Type ABf buffers. Meanwhile, jagged (multidirectional) buffer fracture contours were found much more frequently in Type ABf particles than Type Af particles. Approximately one-third of the 152 particles covered in Figure 18 contained both shapes of buffer fracture, as in Figure 14(a). In addition, 16 Af particles and $17 \mathrm{ABf}$ particles included in Table 2 showed too little of their buffer fractures for contours to be clearly distinguished, so they could not be included in Figure 18.

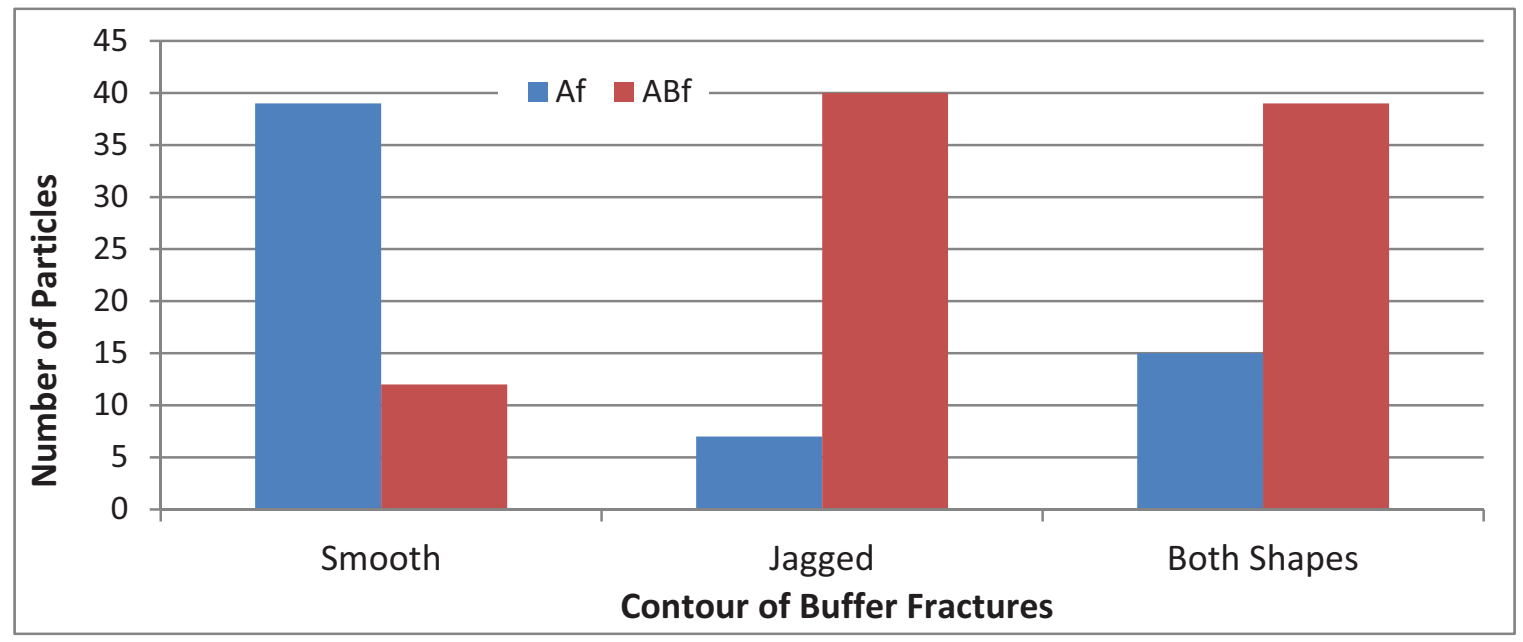

Figure 18. Different buffer fracture shapes seen in Type Af and Type ABf particles.

Determining how jagged buffer fractures initiated is very difficult from end-state particle configurations. Nevertheless, several images of Type AB particles apparently contain rare incipient tangential buffer fractures. Two examples are presented in Figure 19 where the incipient tangential fractures began at the kernel interface and coincide azimuthally with the ends of buffer-IPyC debonds. The bright thickness at the kernel interface is referred to as a uranium carbide "skin" [Hunn et al. 2005]. It may have had some role in initiating the tangential buffer fractures. 

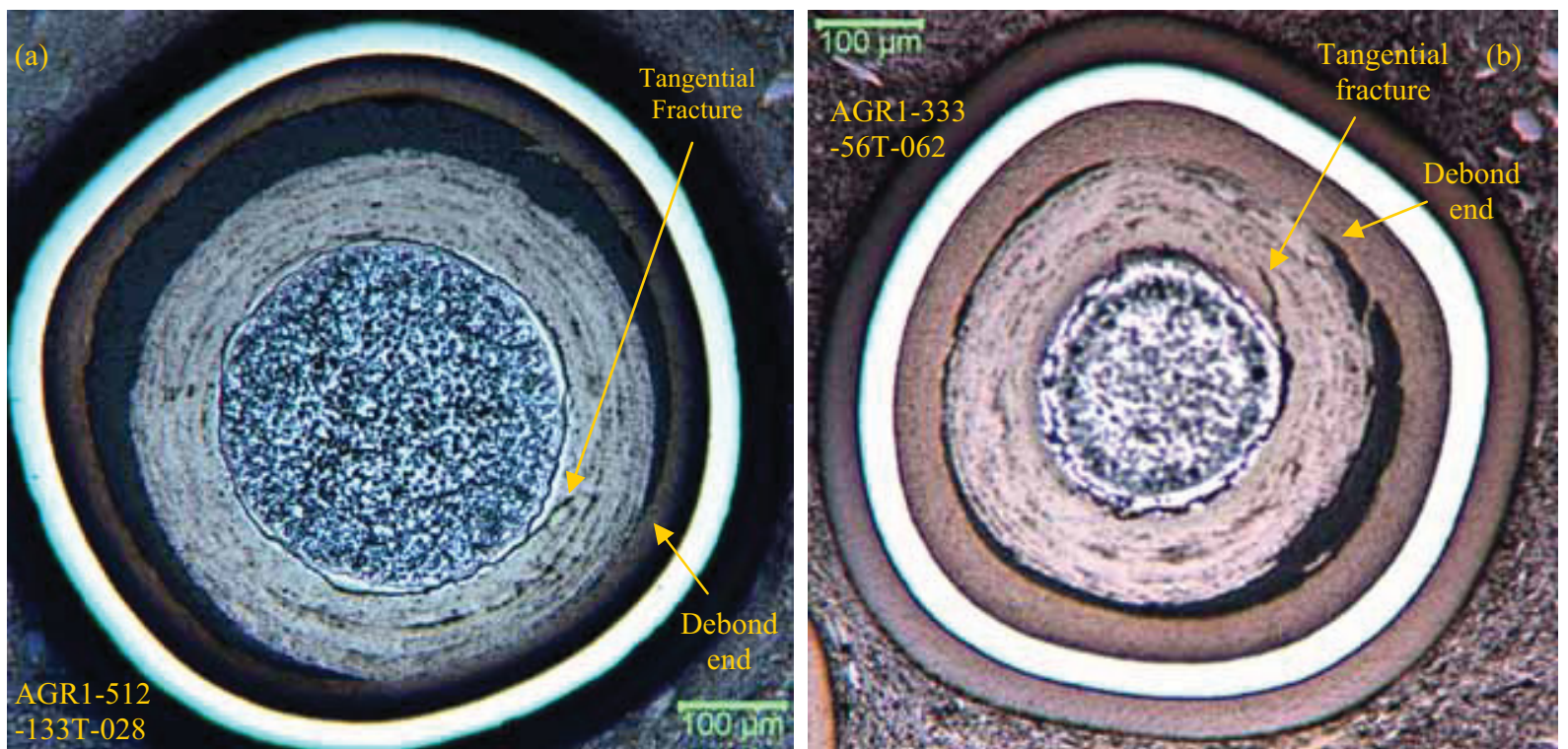

Figure 19. Apparent incipient buffer fractures that initiated tangentially near azimuths where buffer-IPyC debonds terminated.

Figure 20 displays two tangential buffer fractures that progressed beyond the incipient stage. In both of these examples, the tangential buffer fractures split from the kernel interface at approximately the same azimuths as the buffer-IPyC debonds ended. However, the azimuths where the buffers and IPyC layers were separating when the buffer fractures were initiated cannot be determined. Figure 20(a) also reveals an incipient buffer fracture toward the bottom. Here the fracture connected the kernel interface with an apparent concentration of buffer porosity that was circumferentially oriented. Similar circumferential pore concentrations are evident in portions of Figures 19(a) and 19(b), as well.
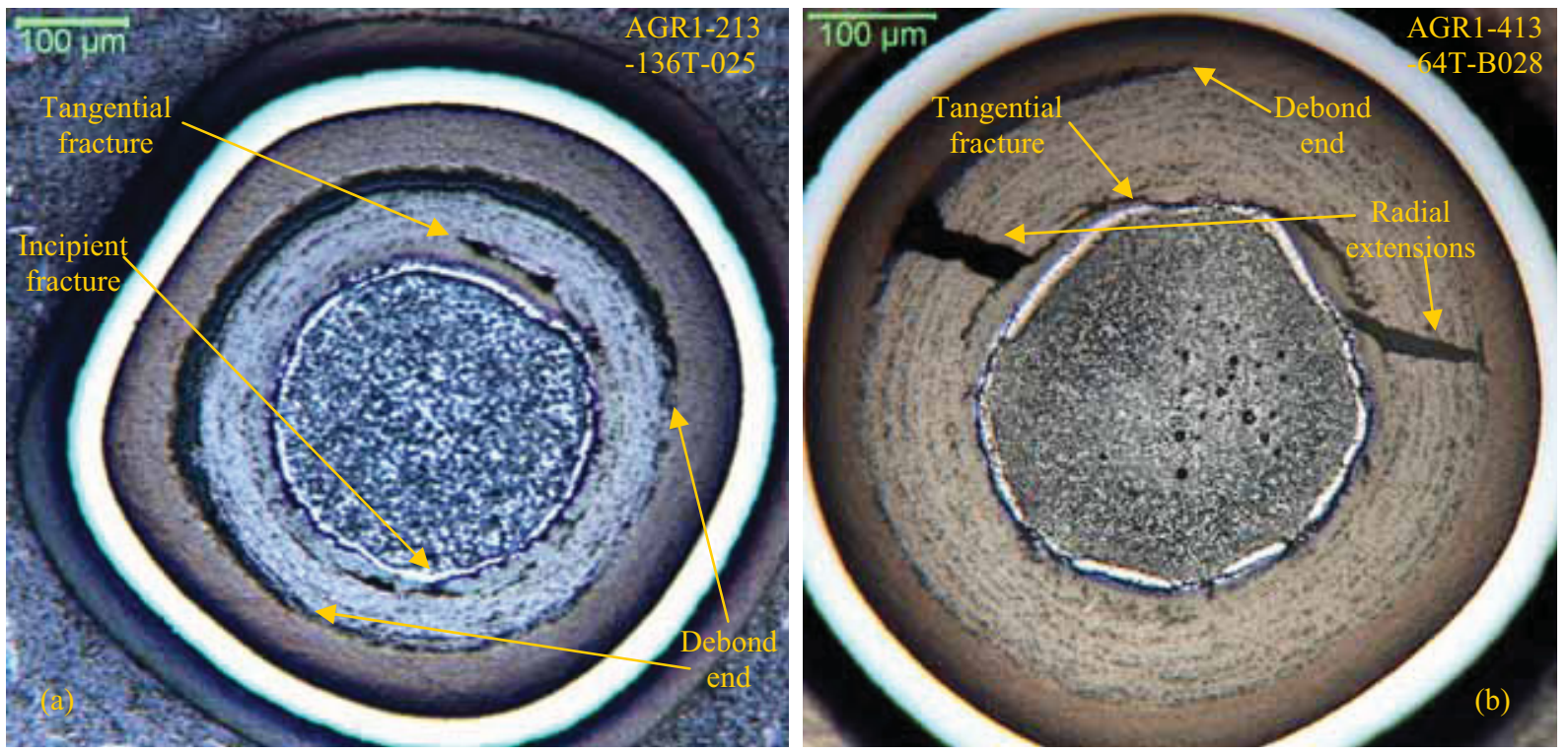

Figure 20. Tangential buffer fractures that propagated to varying extents. 


\subsubsection{IPyC Fractures}

Fractures in irradiated AGR-1 IPyC layers were observed in a range of extents, from incipient to through-wall, as well as in a wide variety of circumstances. Both images in Figure 21 suggest that the IPyC layer may have torn during buffer-IPyC debonding at interfacial points where bonding was especially strong. Locally strong bonding evidently initiated the two short tears in Figure 21(a), but the bonds apparently released at both locations before these fractures penetrated the entire IPyC layer. The tear was much longer where the buffer-IPyC bond was maintained. In Figure 21(b), buffer-IPyC debonding halted at the lower IPyC fracture, but apparently resumed after starting the IPyC fracture at the upper left. These observations infer that bond strength varied considerably along the buffer-IPyC interface, at least within these two Compact 5-1-2 particles. In any case, buffer fractures clearly were not essential for IPyC fractures. Similar behavior with buffer fracturing is shown in Figure 11 for the Type ABf example, where IPyC fractures were found immediately adjacent to the circumferential region that still had a bonded buffer-IPyC interface.
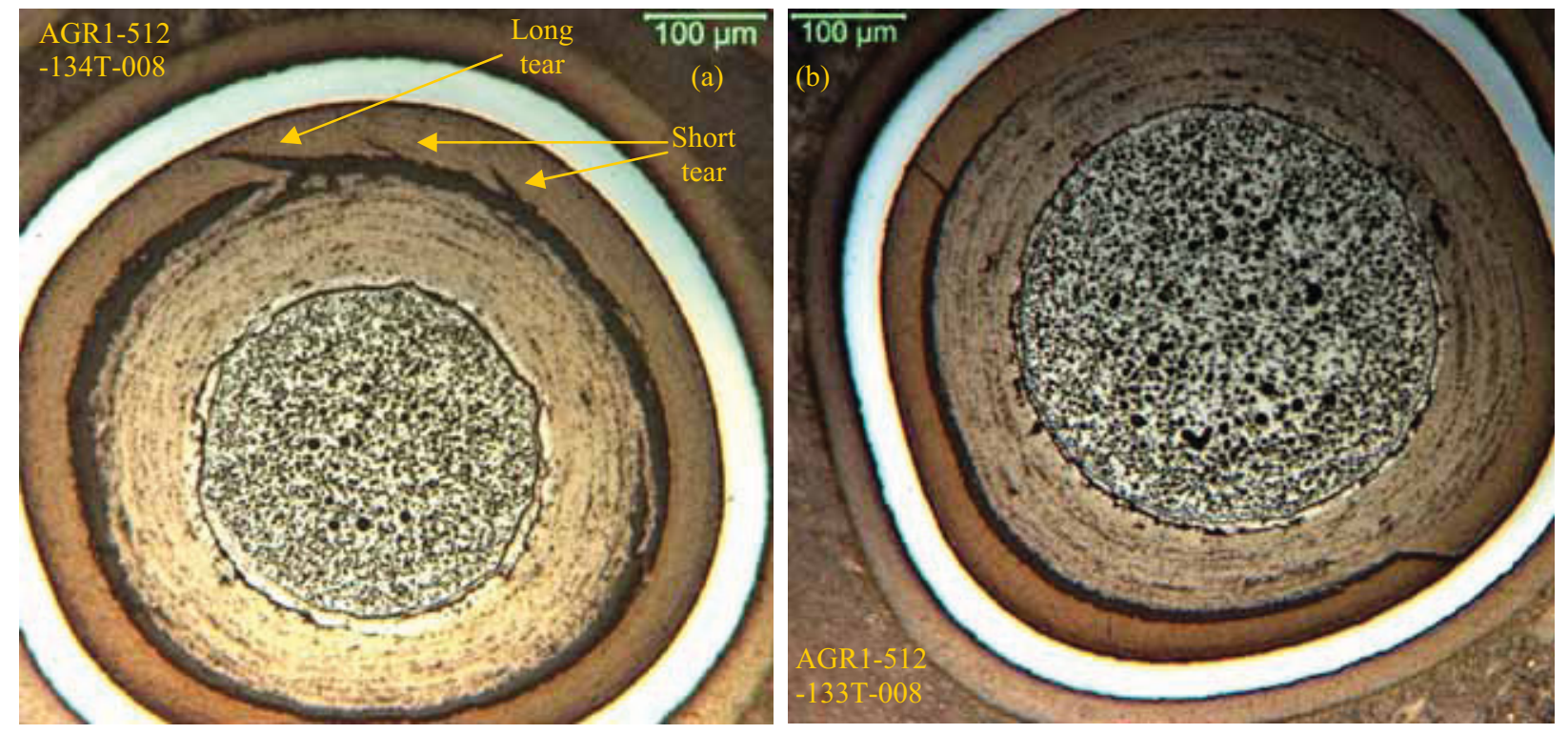

Figure 21. IPyC fractures where buffer apparently was strongly bonded to IPyC layer.

Two Type ABf particles with multiple IPyC fractures are presented in Figure 22. Figure 22(a) shows two IPyC fractures at ends of buffer-IPyC debonds, both of which resemble those in Figure 21(b). The IPyC fracture at the lower left of Figure 22(a) is not aligned with the nearby buffer fracture so the IPyC fracture may not have been caused by the buffer fracture. The buffer fracture in the right side of Figure 22(a) split a Type B-like region where the buffer and IPyC layers were still bonded. The buffer fracture and IPyC fracture clearly aligned here, so this IPyC fracture likely propagated from the adjacent buffer fracture. Figure 22(b) is very similar to Figure 22(a). The IPyC fracture at the top was at the end of a buffer-IPyC debond, well removed from any buffer fractures. The IPyC fracture on the left side of Figure 22(b) penetrated another Type B-like area where the buffer was bonded to the IPyC layer on both sides. A short IPyC-SiC debond occurred at the tip of this IPyC fracture. 

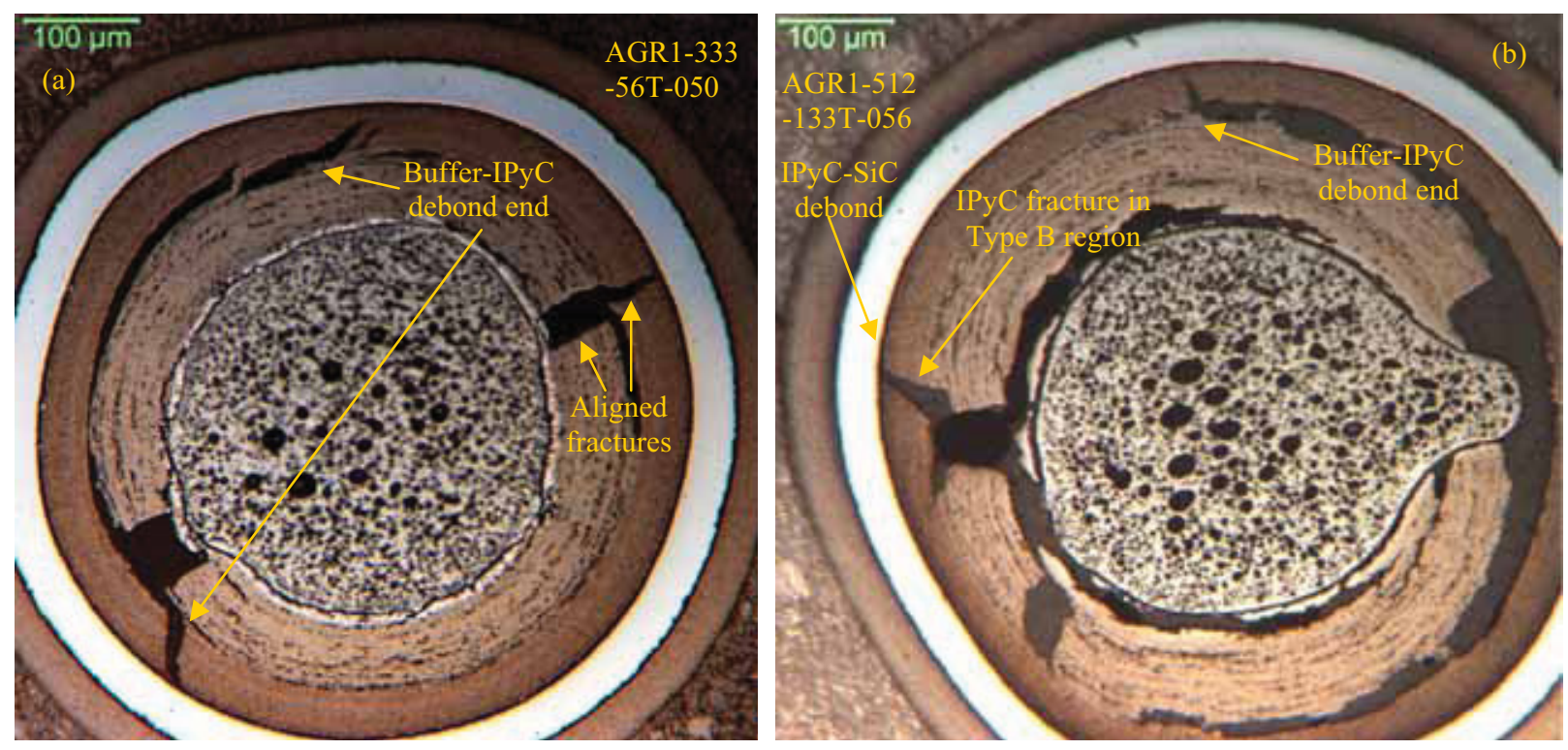

Figure 22. IPyC fractures in Type AB particles with multiple buffer fractures.

Extensive IPyC-SiC debonding is displayed for a Type B particle in Figure 23 at the tips of all three IPyC fractures. These large debonded IPyC-SiC regions extended symmetrically on both sides of the radial IPyC fractures. All three IPyC fractures apparently propagated directly from the radial buffer fractures.

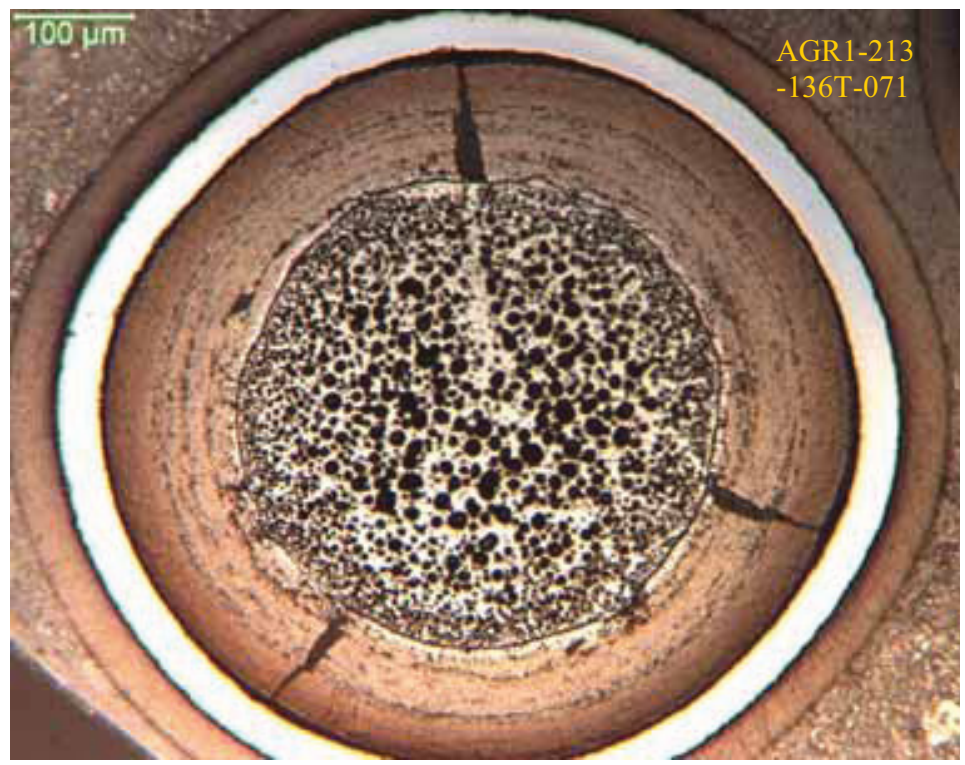

Figure 23. Extensive IPyC-SiC debonding at tips of three IPyC fractures in a Type Bf particle.

IPyC fracture statistics are given in Table 3, where particles with both partial and through-IPyC fractures visible in the plane of polish were counted as particles with the more severe through-IPyC fracture. Minor tears from buffer debonding happened in a small percentage of Types Ai, Af, and ABi particles, while through-IPyC fractures in these types were even rarer. No partial or through-wall IPyC fractures were found in Type Bi particles, but all six Type Bf particles had fractures extending through the entire IPyC layer at azimuths that coincided with buffer fractures. It seems likely that every buffer fracture in a Type B particle will cause a fracture through the entire IPyC layer. 
Table 3. Summary of IPyC fracture data from all classified particles in five AGR-1 fuel compacts.

\begin{tabular}{|c|c|c|c|c|c|}
\hline \multirow{2}{*}{ Particle type } & $\begin{array}{c}\text { Number of } \\
\text { particles in } \\
\text { each type }\end{array}$ & $\begin{array}{c}\text { With partial IPyC fractures } \\
\text { fractures }\end{array}$ & $\begin{array}{c}\text { With fractures through IPyC } \\
\text { frequency }\end{array}$ & $\begin{array}{c}\text { Particles with } \\
\text { fractures }\end{array}$ & $\begin{array}{c}\text { Fracture } \\
\text { frequency }\end{array}$ \\
\hline $\mathrm{Ai}$ & 435 & 7 & $1.6 \%$ & 2 & $0.5 \%$ \\
\hline $\mathrm{Af}$ & 77 & 4 & $5.2 \%$ & 0 & $0.0 \%$ \\
\hline $\mathrm{Bi}$ & 40 & 0 & $0.0 \%$ & 0 & $0.0 \%$ \\
\hline $\mathrm{Bf}$ & 6 & 0 & $0.0 \%$ & 6 & $100.0 \%$ \\
\hline $\mathrm{ABi}$ & 164 & 2 & $1.2 \%$ & 2 & $1.2 \%$ \\
\hline $\mathrm{ABf}$ & 108 & 8 & $7.4 \%$ & 19 & $18.1 \%$ \\
\hline $\mathrm{Total}$ & 830 & 21 & $2.5 \%$ & 29 & $3.5 \%$ \\
\hline
\end{tabular}

Table 3 shows that both partial and especially through-wall IPyC fractures were much more likely in Type ABf particles than in Type ABi particles. IPyC fractures that propagated directly from a buffer fracture, such as in the Type B-like regions in Figure 22, contributed a bit to this trend. However, most IPyC fractures in Type ABf particles were located far from buffer fractures, as shown in the Type ABf example in Figure 11. Instead of buffer fractures causing most IPyC fractures in Type ABf particles, locally strong buffer-IPyC bonding may have been involved in initiating fractures in Type AB buffers as well as in initiating IPyC fractures. Note that Type AB buffer fractures and IPyC fractures could have initiated at different times at different azimuths as the apparently uneven buffer-IPyC bond delaminated along an interface, which complicates interpretations of end-state configurations in micrographs.

Figure 24 presents the frequencies of IPyC fractures among the five AGR-1 compacts. Results vary substantially, particularly for fractures that penetrated the entire IPyC layer. Although values were similar for Compacts 2-1-3 (Variant 2), 3-3-3 (Baseline), and 6-3-3 (Baseline), particles in Variant 1 Compact 5-1-2 contained a much higher incidence of through-IPyC fractures (19 of 205 classified particles). The higher frequency of through-IPyC fractures in Compact 5-1-2 may be related to the lower IPyC density from the elevated IPyC deposition temperature in Variant 1 fuel [Hunn and Lowden 2006-1]. Zero through-IPyC fractures were found in Variant 3 Compact 4-1-3 (157 classified particles) despite its higher irradiation temperatures and neutron exposure (see Table 1).

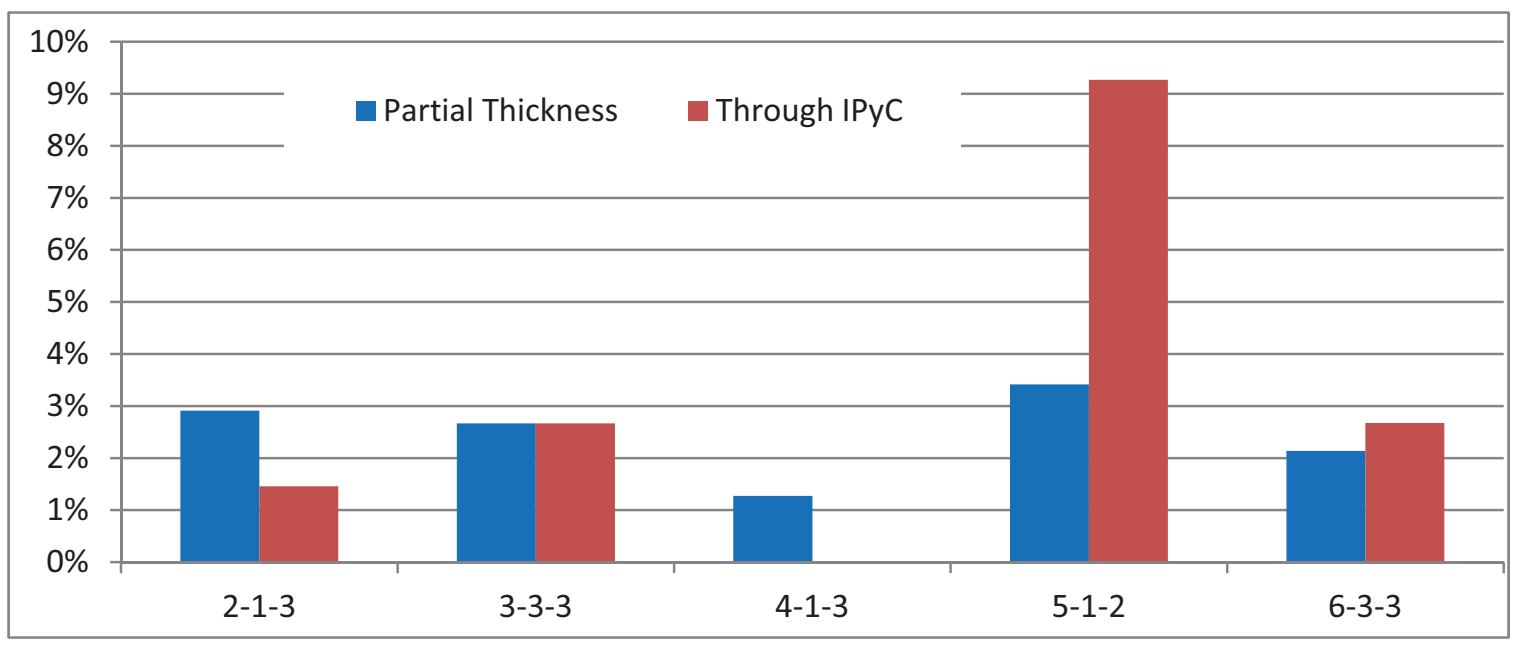

Figure 24. Percentages of particles with IPyC fractures among irradiated AGR-1 compacts. 


\subsubsection{SiC Fractures}

$\mathrm{SiC}$ fractures were found in only five irradiated AGR-1 particles from the five cross-sectioned fuel compacts, including two unclassified particles without visible kernels. None of these fractures were determined to have breached the entire $\mathrm{SiC}$ layer thickness during irradiation.

Two cross-sectioned particles exhibited abnormal SiC structure and appeared to contain foreign material between the IPyC and $\mathrm{SiC}$ layers. Both of these particles also had $\mathrm{SiC}$ fractures. The first particle is displayed in Figure 25. The lenticular bulge between the IPyC exterior and $\mathrm{SiC}$ interior on the right side is characteristic of a soot inclusion, which occurs during fabrication when over-fluidized particles pick up carbon soot from the walls of the coating chamber. A soot inclusion can result in a region of porous $\mathrm{SiC}$ where SiC infiltrates the low density soot during deposition. This anomaly has been termed a "gold spot" defect because of the optical appearance of the defective $\mathrm{SiC}$ after the OPyC layer has been removed. Particles with soot inclusions were observed during characterization of the preirradiated fuel particles with defect frequencies equal to 1-4 particles per compact [Hunn and Lowden 2006-2]. The tangentially oriented $\mathrm{SiC}$ fractures in Figure 25 apparently started where the IPyC and $\mathrm{SiC}$ were allowed to bond by the absence of foreign material. Radial stress from IPyC densification would be expected to concentrate at these positions and induce tensile stress in the SiC layer [Miller et al. 2004]. Buffer and IPyC layers stayed bonded around the entire interface in this Type Bf particle. The fracture through the buffer and IPyC layer on the left side of this particle was not clearly caused by the foreign material. The aligned buffer-IPyC fracture in this particle resembles those in Figure 23 with two exceptions. The kernel swelled into the buffer cavity, which was narrower than most buffer cavities in Type Af and Type ABf particles and the IPyC-SiC debond at this crack tip was much shorter than at all three crack tips in Figure 23.

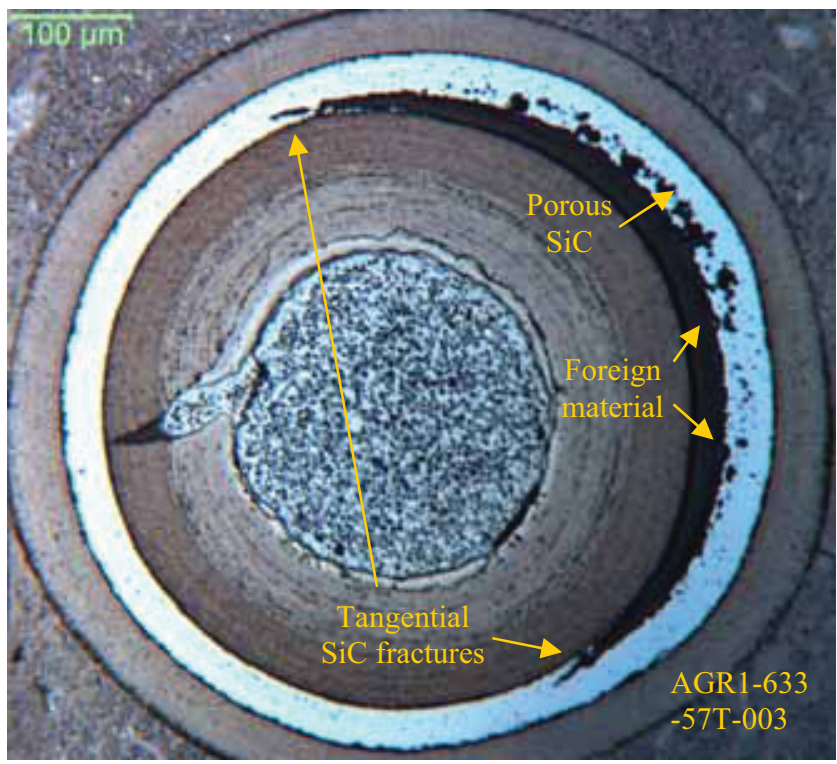

Figure 25. Tangential SiC fractures bordering a probable soot inclusion.

The second particle with $\mathrm{SiC}$ fractures and foreign material is shown in Figure 26. This particle was not classified because of the absence of a visible kernel in the section examined. As in Figure 25, there was a distinct bulge between the IPyC and $\mathrm{SiC}$ on the right side. The $\mathrm{SiC}$ fractures began in a tangential direction, but here they propagated farther into the $\mathrm{SiC}$ and abruptly veered in the radial direction. The radial extensions probably occurred during grinding of this mount because no cesium-134 was detected in the Capsule 2 graphite fuel holder, whereas release of cesium fission products would have been anticipated had the SiC layer failed completely during irradiation [Harp and Ploger 2011]. 


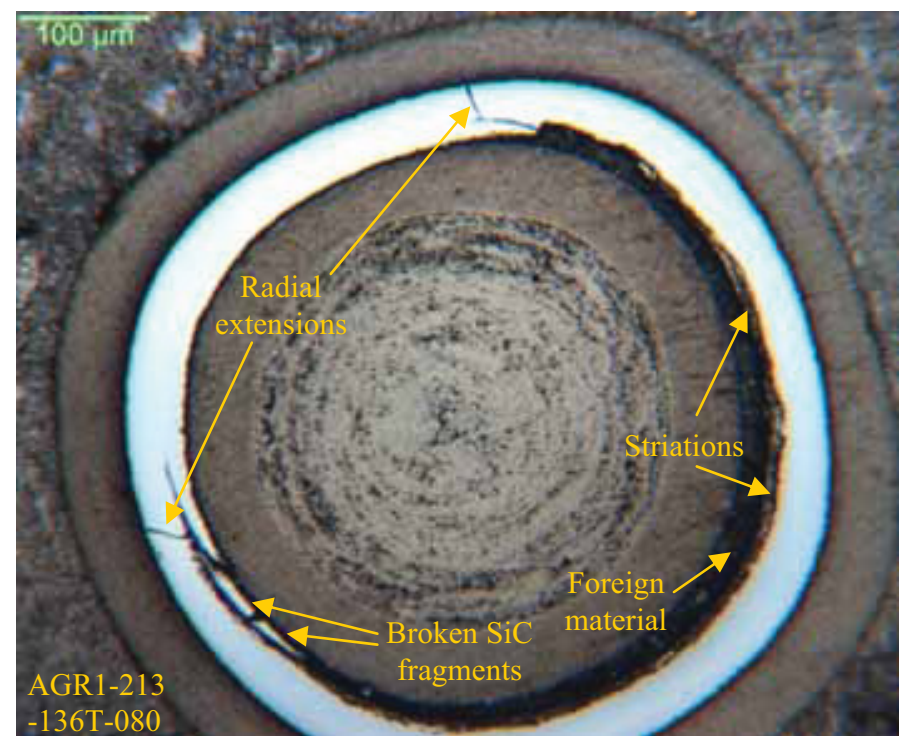

Figure 26. SiC fractures in the crown of a particle with foreign material between IPyC and SiC.

Unlike the particle in Figure 25, the $\mathrm{SiC}$ interior in Figure 26 was not pitted but rather showed circumferential striations. Because this particle was not ground close to the $\mathrm{SiC}$ midplane, the striations were only in the thinnest portion of the $\mathrm{SiC}$ where it was undercut by the foreign material. These striations most likely occurred during grinding and probably reflect poor underlying support. The two fragments of thin $\mathrm{SiC}$ inside the tangential fracture at the lower left of Figure 26 probably broke off during grinding due to poor underlying support, as well.

Fractures partway through the $\mathrm{SiC}$ layer were discovered in three other irradiated particles from these five compacts. There is no indication that any foreign material was present between the IPyC and $\mathrm{SiC}$ layers in these particles. The particle with the most $\mathrm{SiC}$ fractures is presented in Figure 27. It strongly resembles the Type $\mathrm{Bf}$ particle in Figure 23 except for the pair of $\mathrm{SiC}$ fractures that were symmetrically located around each of the two buffer-IPyC fractures. Propagation of the rightmost SiC fracture may have stopped when IPyC-SiC debonding progressed beyond this azimuth toward the top of this image.

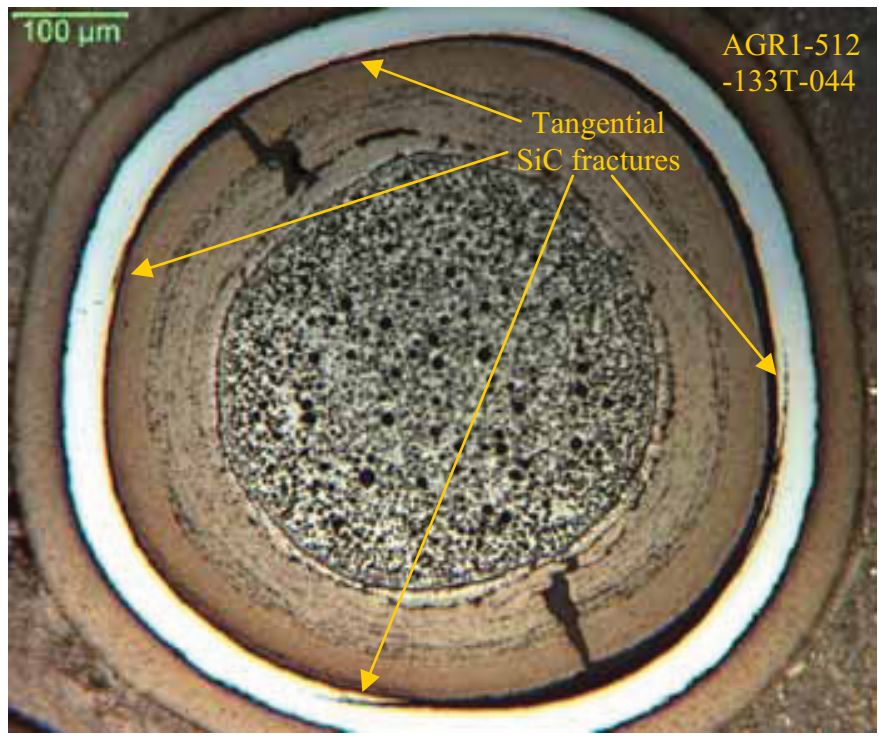

Figure 27. Pairs of SiC fractures symmetrically positioned around two buffer-IPyC fractures. 
The SiC fracture in Figure 28(a) is in yet another Type Bf particle. IPyC and SiC layers debonded along most of the interface at this plane. The $\mathrm{SiC}$ fracture apparently began where the IPyC-SiC bond was still intact. However, IPyC-SiC debonding might not always have been essential for a $\mathrm{SiC}$ fracture, as shown at the crown of an unclassified particle in Figure 28(b). In the absence of conspicuous IPyC-SiC debonding at this plane, the IPyC fracture may have propagated directly into a tangential $\mathrm{SiC}$ fracture.
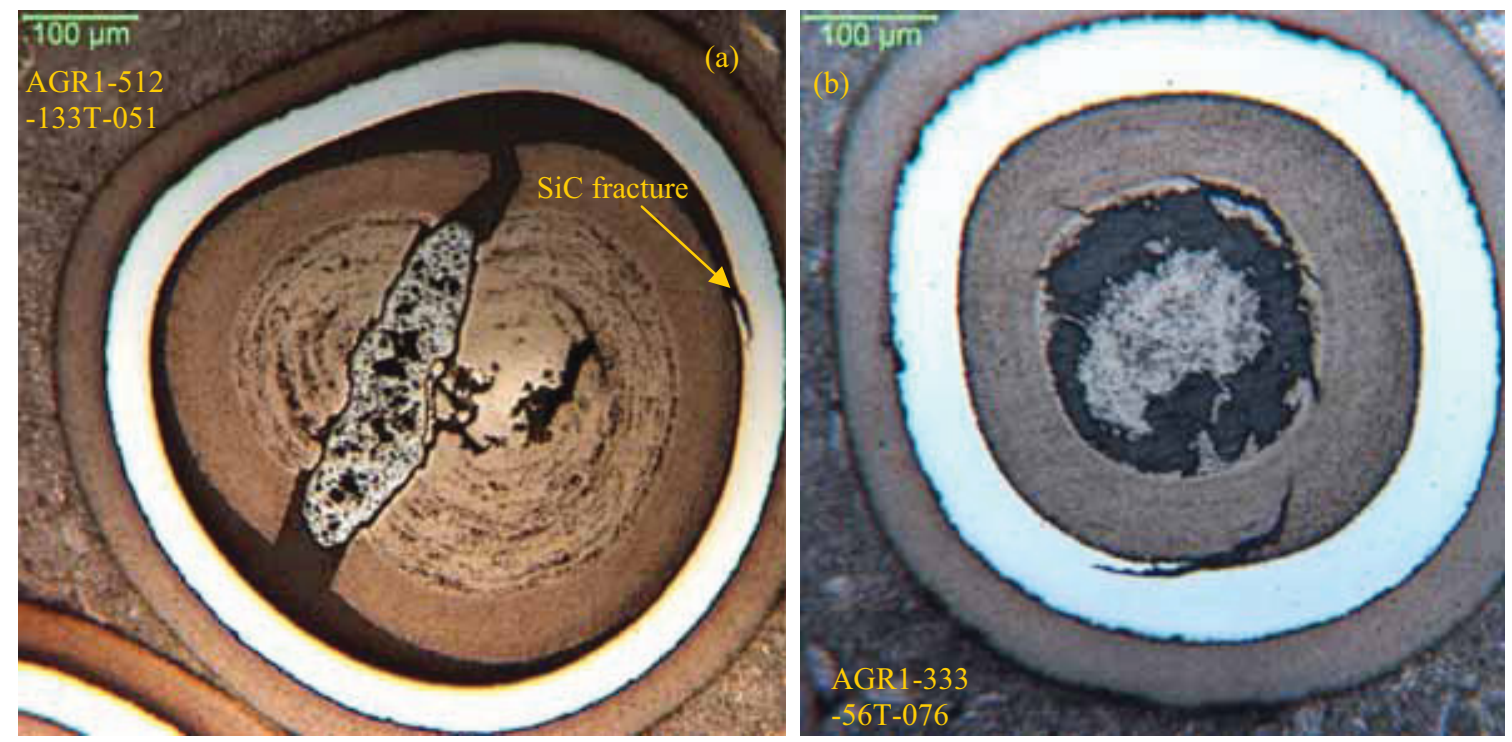

Figure 28. Tangential SiC fractures in two particles whose cross sections are located far from midplane.

Figure 29 examines relationships between major anomalous IPyC and SiC structures in all six types of classified AGR-1 particle. Here the classified particle in Figure 25 that contained foreign material is grouped with other Type Bf particles. (Unclassified particles in Figures 26 and 28(b) could not be included in this figure.) Figure 29 show that Type Bf particles were far more likely to contain IPyC fractures, IPyC-SiC debonds, and $\mathrm{SiC}$ fractures than other types. Although Type $\mathrm{Bf}$ particles represented less than $1 \%$ of the classified particles, they contained nearly $40 \%$ of all the IPyC-SiC debonds and $100 \%$ of the SiC fractures. The absence of SiC fractures in the eight Type ABf particles with IPyC-SiC debonds may be related to the short extents of debonding found at the tips of the IPyC fractures.

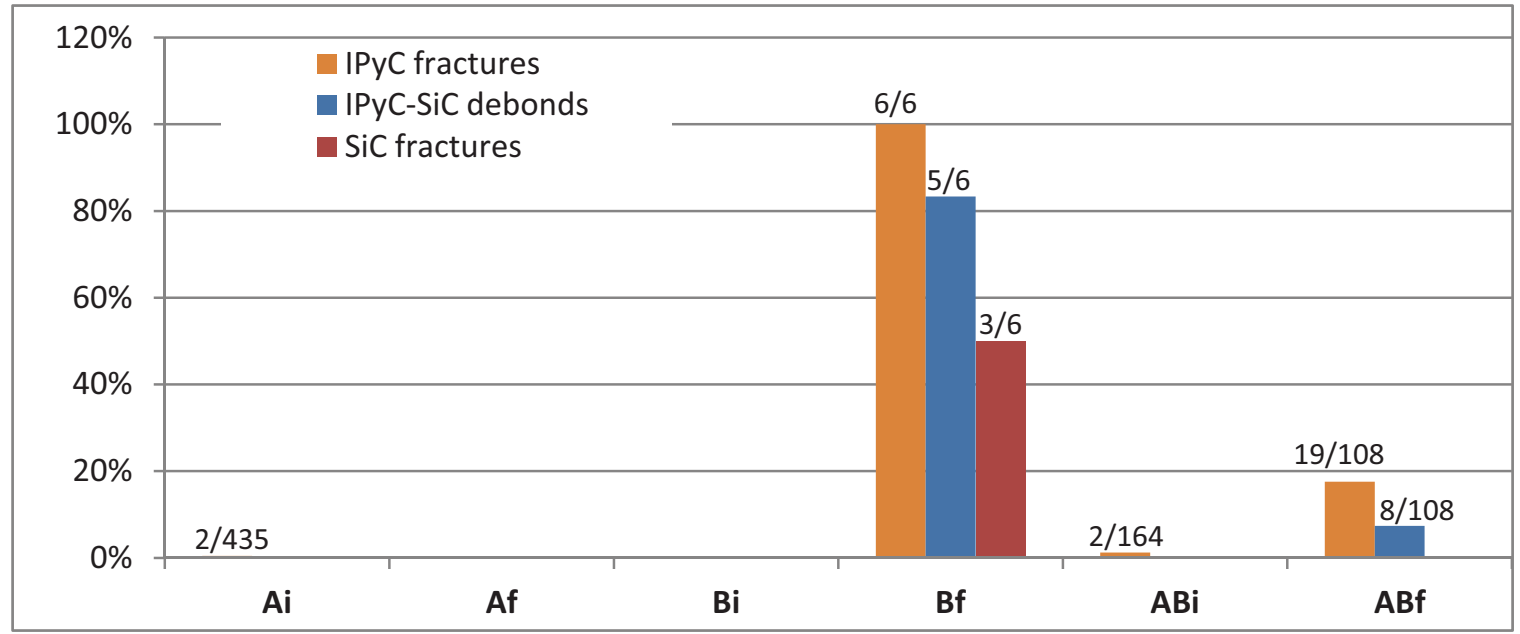

Figure 29. Frequencies of IPyC fractures, IPyC-SiC debonds, and SiC fractures by type among the 830 classified AGR-1 particles. 
IPyC-SiC debonds and $\mathrm{SiC}$ fractures within the five compacts are grouped in Table 4, including the unclassified particles in Figures 26 and 28(b). Table 4 suggests that $\mathrm{SiC}$ anomalies were more frequent in Compact 5-1-2 (Variant 1). This finding is likely related to the much larger frequency of IPyC fractures in Compact 5-1-2 (see Figure 24). The particles with foreign material were too rare for correlation with coating variations. Nevertheless, although only found in rare instances, foreign material evidently was a significant source of $\mathrm{SiC}$ fractures. As noted above, this likely is a result of foreign material-bearing particles being fabricated with extensive IPyC-SiC debonds that caused tensile stress in their $\mathrm{SiC}$ layers.

Table 4. SiC anomalies in AGR-1 compacts, with unclassified particles in Figures 26 and 28(b) included.

\begin{tabular}{|c|c|c|c|c|}
\hline \multirow{2}{*}{ Compact } & \multicolumn{2}{|c|}{ Normal particles } & \multicolumn{2}{c|}{ Foreign material } \\
\cline { 2 - 5 } & $\begin{array}{c}\text { With IPyC-SiC } \\
\text { debonding }\end{array}$ & With SiC fracture & $\begin{array}{c}\text { With IPyC-SiC } \\
\text { debonding }\end{array}$ & With SiC fracture \\
\hline $2-1-3$ & 2 & 0 & 1 & 1 \\
\hline $3-3-3$ & 0 & 1 & 0 & 0 \\
\hline $4-1-3$ & 0 & 0 & 0 & 0 \\
\hline $5-1-2$ & 9 & 2 & 0 & 0 \\
\hline $6-3-3$ & 1 & 0 & 1 & 1 \\
\hline
\end{tabular}

\subsection{Kernel Behavior}

\subsubsection{Kernel Swelling}

The most conspicuous sign of kernel swelling was growth into fractured buffer cavities, as already shown in Figures 14(a), 16, 17(b), 22(b), 25, and 28(a). Cavities in Figures 25 and 28(a) were narrow, which infers that kernels expanded into small spaces if those were the only open spaces available. Kernel growth could be extensive in the absence of buffer constraint, especially on planes were buffer pieces are separated as shown for an extreme example in Figure 30. Alternatively, Figure 31 displays two of several cases where kernel growth into buffer cavities was partially blocked by pieces of buffer material (at least in the plane of polish). In all cases, the amount of constraint imposed by the adjacent buffer clearly had appreciable influence on local kernel growth.

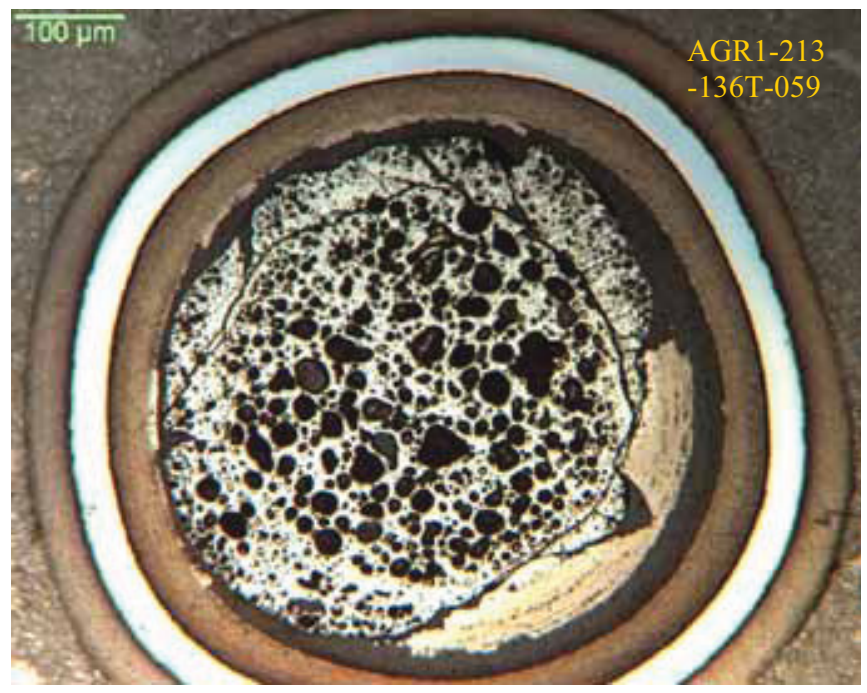

Figure 30. Extensive kernel swelling at a plane between separated pieces of buffer. 

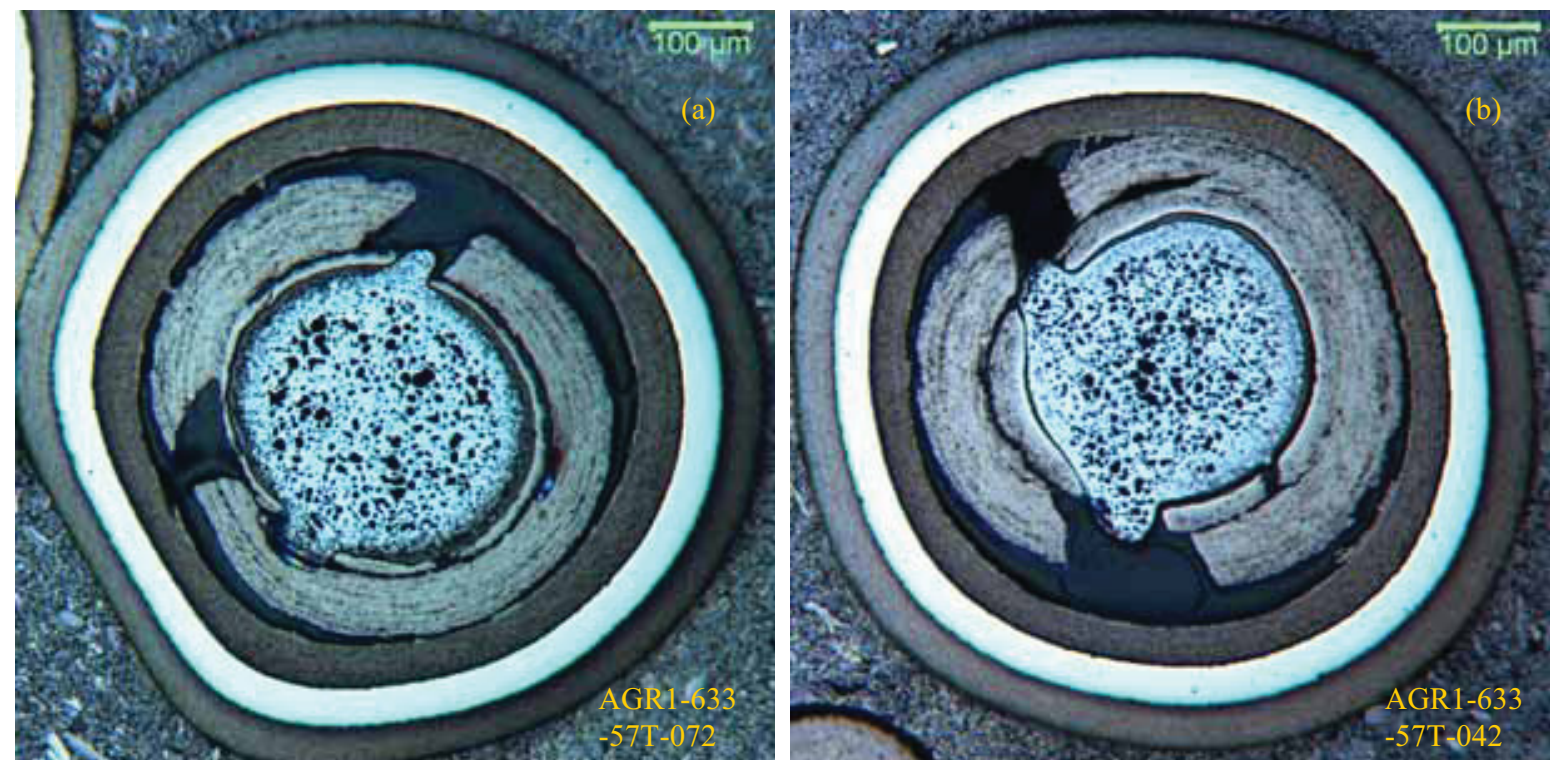

Figure 31. Kernels inhibited from swelling into buffer cavities by buffer fragments.

The left side of Figure 30 shows some discoloration along the interior surface of the IPyC layer where the swelled kernel came in close proximity. A few other particles with fractured buffers displayed more conspicuous evidence of kernel effects on the IPyC layer, and two of the best examples are presented in Figure 32. The IPyC damage evidently was confined to fine porosity that caused the darker appearance. The IPyC tear on the right side of Figure 32(b) appears unrelated, so the structural integrity of the IPyC layer probably was not compromised by the locally extensive kernel swelling.
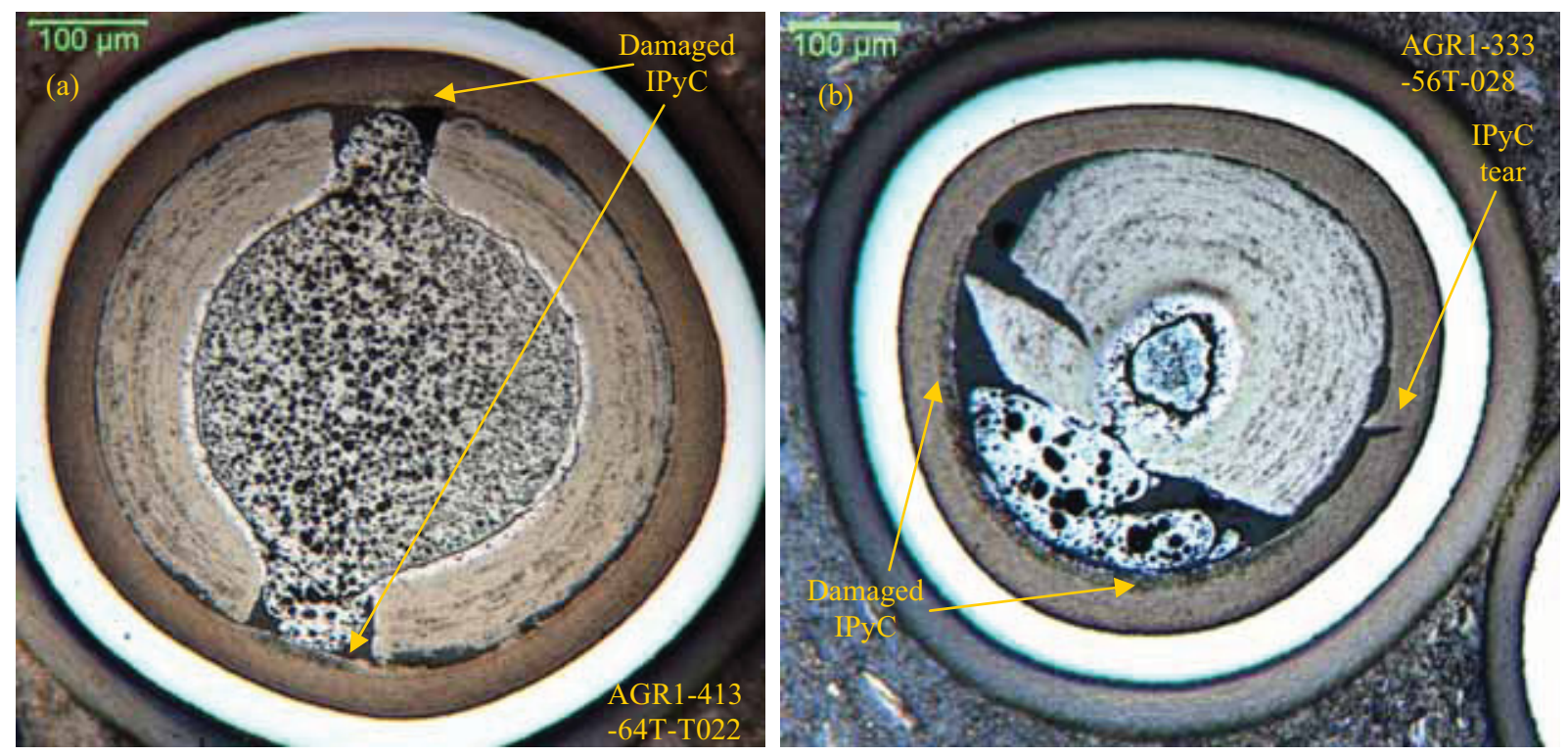

Figure 32. IPyC interiors damaged by kernel growth through fractured buffer cavities.

As discussed in Section 3.1, uniformly strong buffer-IPyC bonding apparently caused buffers in Type B particles to densify radially outward, rather than radially inward as in Type A and Type AB particles. Preliminary modeling results suggest that buffer densification occurred relatively early (in response to fast fluence) while kernel swelling is suspected somewhat later (in response to burnup). If so, a temporary kernel-buffer gap may have formed in Type B particles during some portion of the AGR-1 
experiment. By the end of the AGR-1 irradiation, the vast majority of Type B kernels had swelled sufficiently to close any radial gap, as demonstrated in Figures 11, 23, 25, and 27. However, an apparent residual kernel-buffer gap was found in a small number of Type B particles from Compact 6-3-3, as displayed for two examples in Figure 33. Although the uranium carbide skin in both particles broke out intermittently during sample preparation, especially as shown in Figure 33(b), enough of the bright layer remained in both images to confirm that the buffer interiors were not totally removed and that the apparent kernel-buffer gaps were not entirely due to preferential grinding relief. The kernel in Figure 33(b) does not appear to be surrounded by epoxy, so it may have shifted to a nonconcentric elevation, exaggerating the gap thickness.
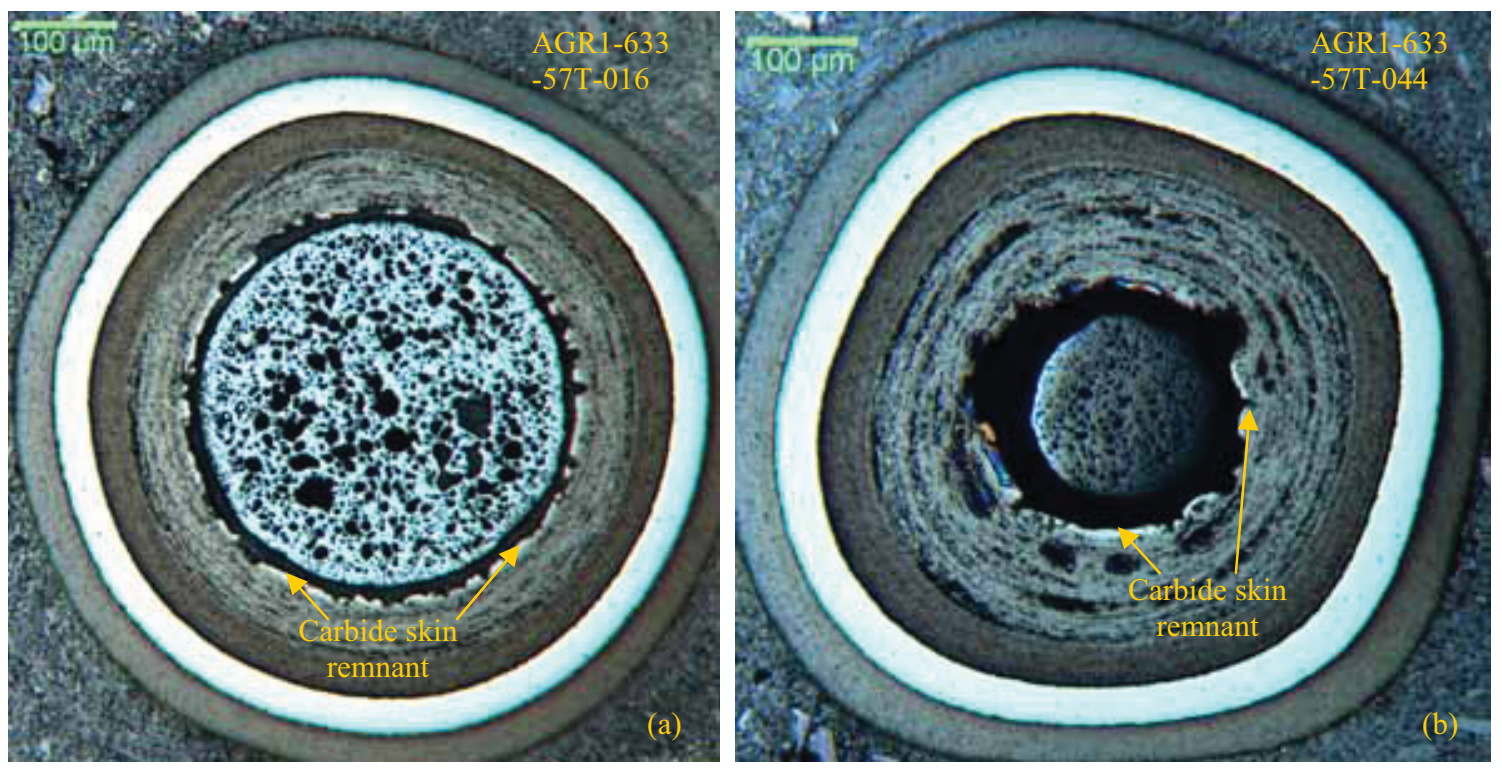

Figure 33. Apparent radial gap between kernel and buffer in two Type B particles from Compact 6-3-3.

\subsubsection{Pore Enlargement}

Kernel growth into buffer cavities often was accompanied by pore enlargement. In a side-by-side comparison, Figure 34 contrasts the larger kernel pores inside a fractured buffer to pores inside an intact buffer. This suggests that more pore enlargement occurred in the Particle 063 kernel after buffer fracturing than in Particle 067 (surrounded by an intact buffer). Figure 34 suggests that many small pores coalesced into fewer large pores after buffer fracture, but some pore expansion likely occurred in response to diminished external pressure on the kernel after buffer fracture, as well. (Such side-by-side comparisons can be misleading because as-fabricated microstructures of AGR-1 kernels were not identical.) Pore sizes were not always so different inside fractured and intact buffers, even within this same fuel compact, as demonstrated in Figure 35. One possible explanation is that the buffer in Particle 063 may have fractured earlier than the buffer in Particle 032, which is also supported by observing more kernel growth into buffer cavities in Particle 063 than in Particle 032. Growth in all four kernels in these two figures would have been constrained to nearly the same extent until buffer fracturing occurred. 


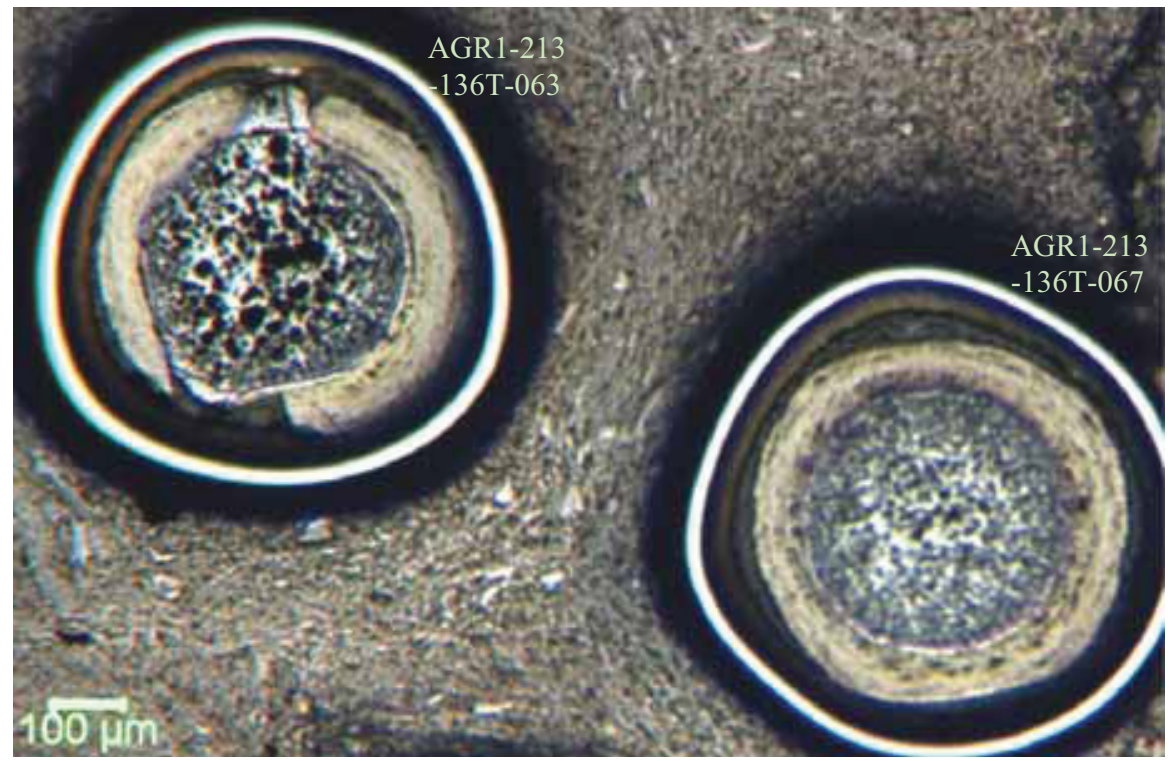

Figure 34. Larger kernel pores inside a fractured buffer than in a nearby kernel inside an intact buffer.

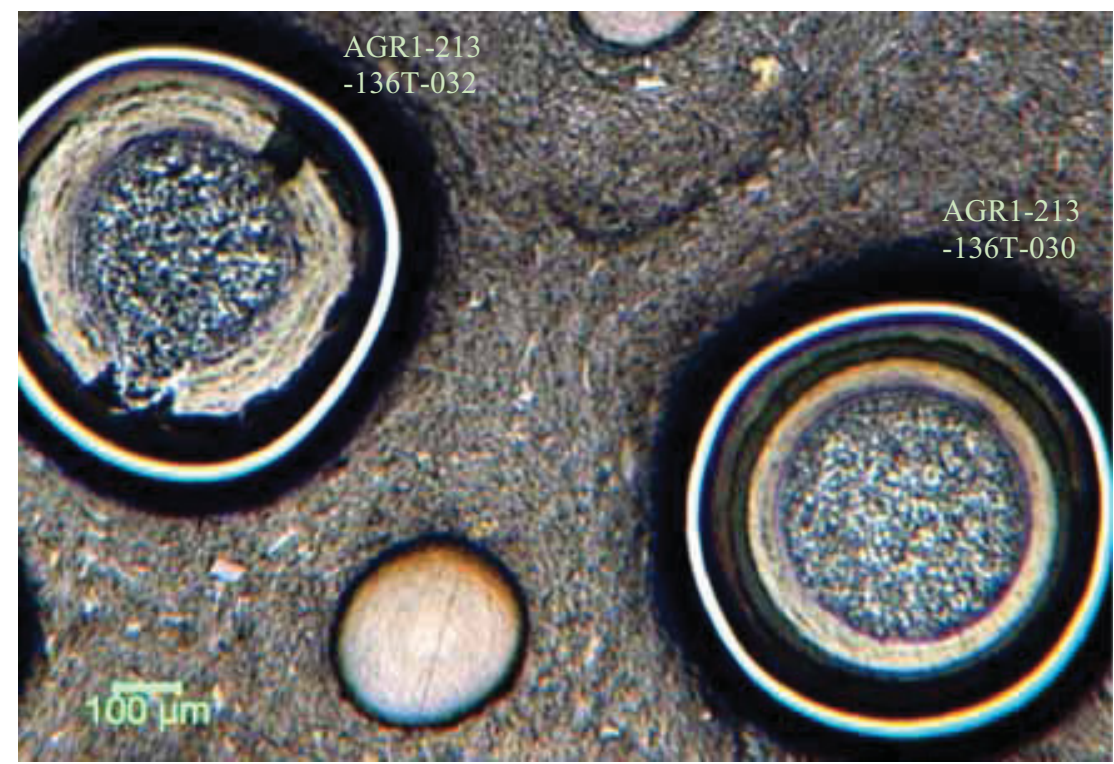

Figure 35. Kernel pores within a fractured buffer that were only slightly larger than inside an intact buffer.

Figure 36 provides two side-by-side examples (also from Compact 6-3-3 Mount 57T with a high concentration of Type B particles) where pores were larger in Type B particles than in nearby particles with fractured buffers. The additional pore enlargement in Type B particles probably reflects even less constraint on kernel swelling than imposed by fractured buffers. Type B buffers remained bonded to IPyC layers and densified outward, imposing little or no constraint on kernels throughout the AGR-1 experiment. Buffer fracturing was not necessary for substantial pore enlargement in both Type B particles. 

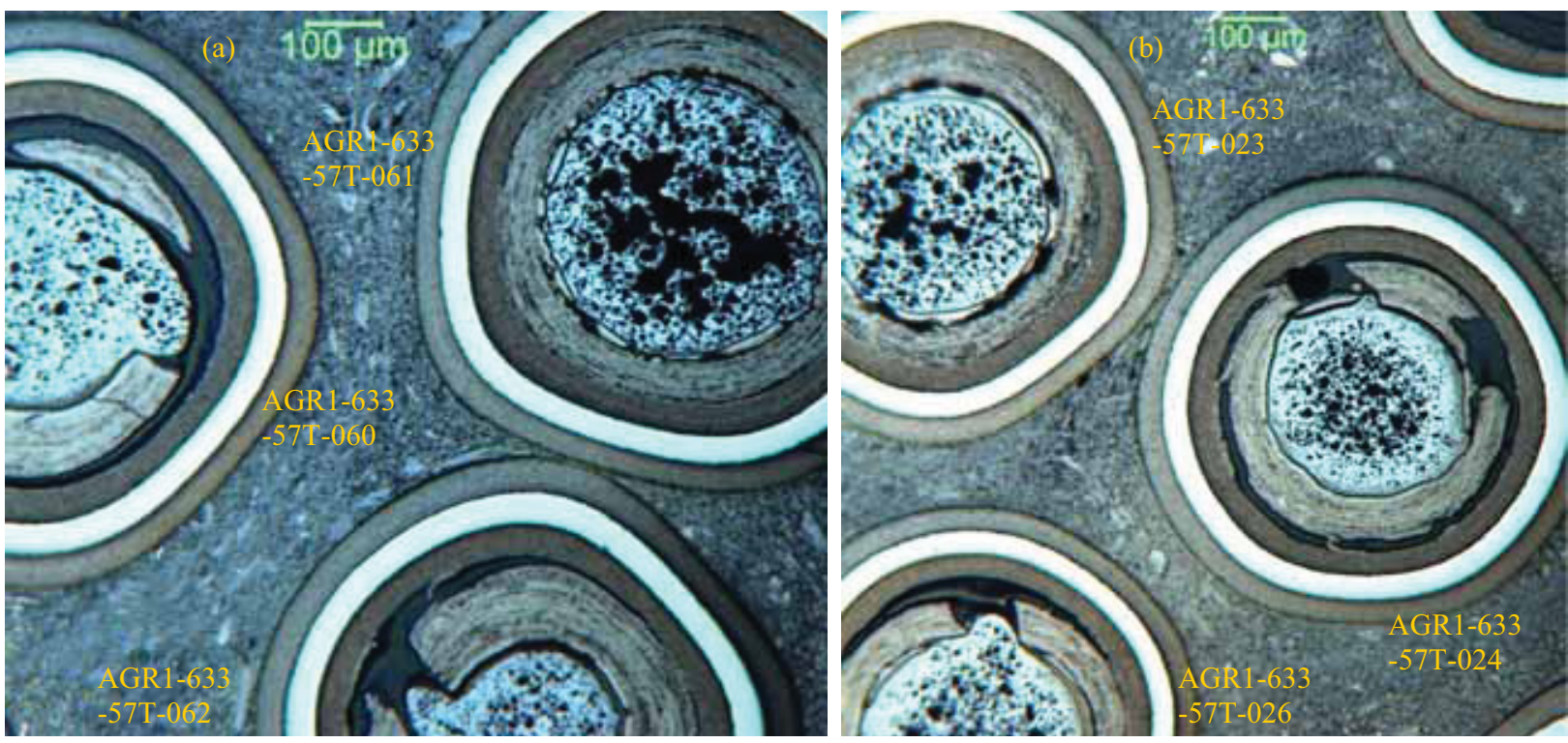

Figure 36. Larger pores in Type Bi Particles 061 and 023 than in nearby particles with fractured buffers.

The largest pores generally were located toward the kernel centers. In some particles with fractured buffers, such as in Figure 17, larger pores could also be found where kernels swelled into buffer cavities. A small number of kernels exhibited asymmetrical, localized pore enlargement, which may be related to microstructural variations in as-fabricated kernels. Two kernels with such concentrations of large pores are shown in Figure 37.
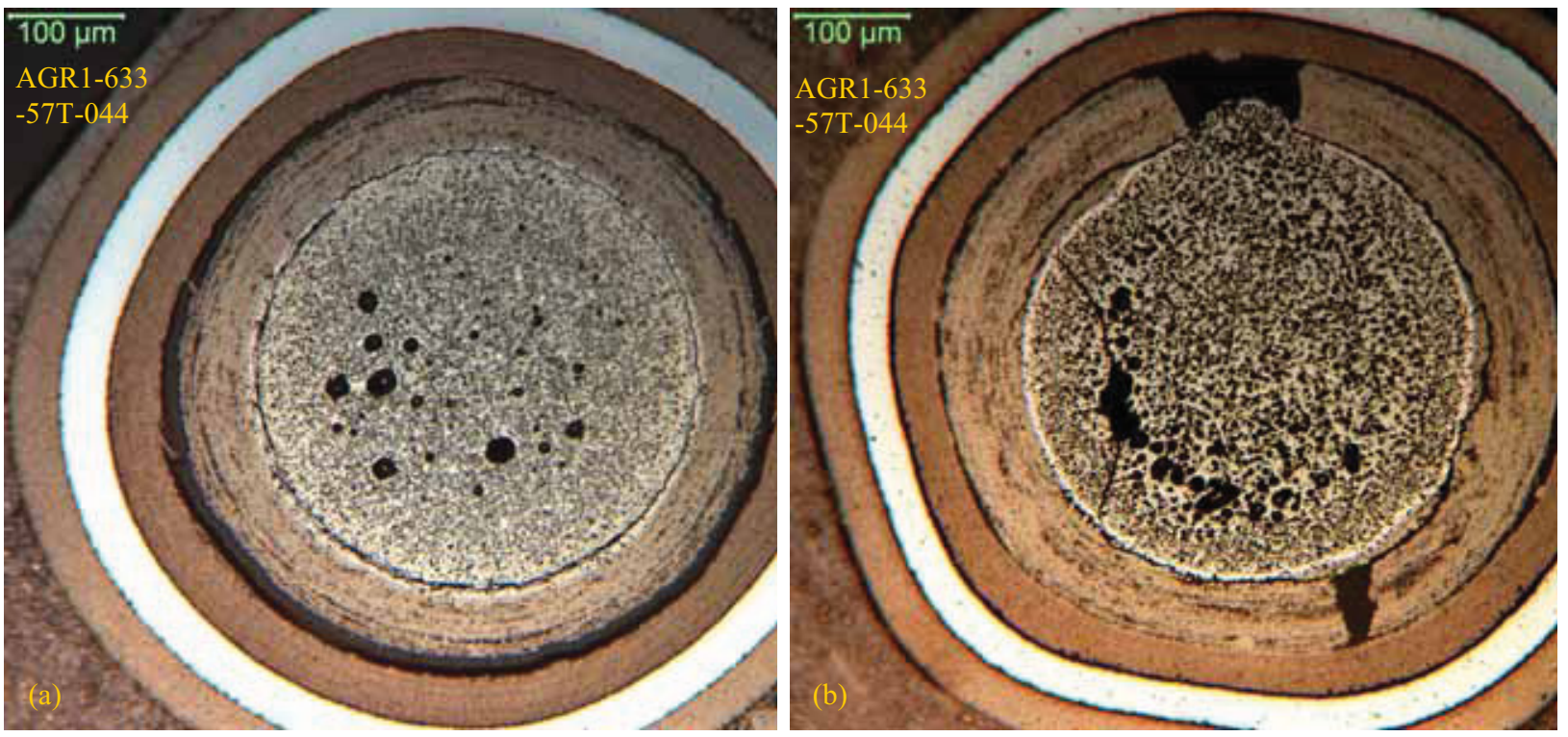

Figure 37. Two examples of spatial variations in pore enlargement. 


\section{PARTICLE MEASUREMENT RESULTS}

Measurements were made on a total of 95 calibrated particle images from three irradiated AGR-1 compacts. The transverse cross sections of Compacts 2-1-3, 4-1-3, and 6-3-3 were chosen to span the estimated ranges of burnup and temperature for the AGR-1 experiment (see Table 1). To minimize geometric errors only those particles ground within $15 \mu \mathrm{m}$ of the nominal $\mathrm{SiC}$ midplane radius were measured, which eliminated approximately $60 \%$ of the particles with exposed kernels in the three mounts. However, this screening did not guarantee that kernels and buffers were ground near midplane because of the buffer-IPyC gap that formed from buffer densification in most particles, which often resulted in major deviations from concentricity. This uncertainty in kernel/buffer center elevations combined with the substantial variation in as-fabricated sizes prevented accurately estimating irradiation-induced changes in kernel and buffer volumes (see Recommendations). Despite the limitations on obtaining accurate quantitative values, dimensions of key features were extracted from cross-sectional images to investigate overall trends among the three compacts as well as among different particle morphologies.

All measurements were made using PAX-it image processing software (Midwest Information Systems, Inc.). As indicated on the examples in Figure 38, circles were fit to manually selected points along $\mathrm{SiC}$ exteriors for a radius-based determination of proximity to midplane (rather than along than along the somewhat rounded, poorly defined OPyC exteriors). Circles were also fit to intact buffer layer exteriors and interiors for estimating average buffer thickness and to IPyC interiors for determining average buffer-IPyC gap thickness. In particles with fractured buffers, buffer thickness was calculated as the difference between arc radii. Porosity was measured as a percentage of kernel area, where a consistent gray level threshold was used for all particles. (Low porosity kernel exteriors were excluded from porosity measurements to avoid a second threshold; see Recommendations.) Polygons were traced around kernels in order to determine the kernel area and to calculate kernel area increase (2-D indication of volumetric swelling), around the largest pore or interconnected pores for an indicator of pore enlargement (area converted to equivalent diameter), and around buffer boundaries to calculate area decrease. As discussed below, results sometimes were analyzed according to morphological types or groupings thereof. These characteristic morphologies were defined in Figure 11, where particular attention was paid to buffer-IPyC bonding.
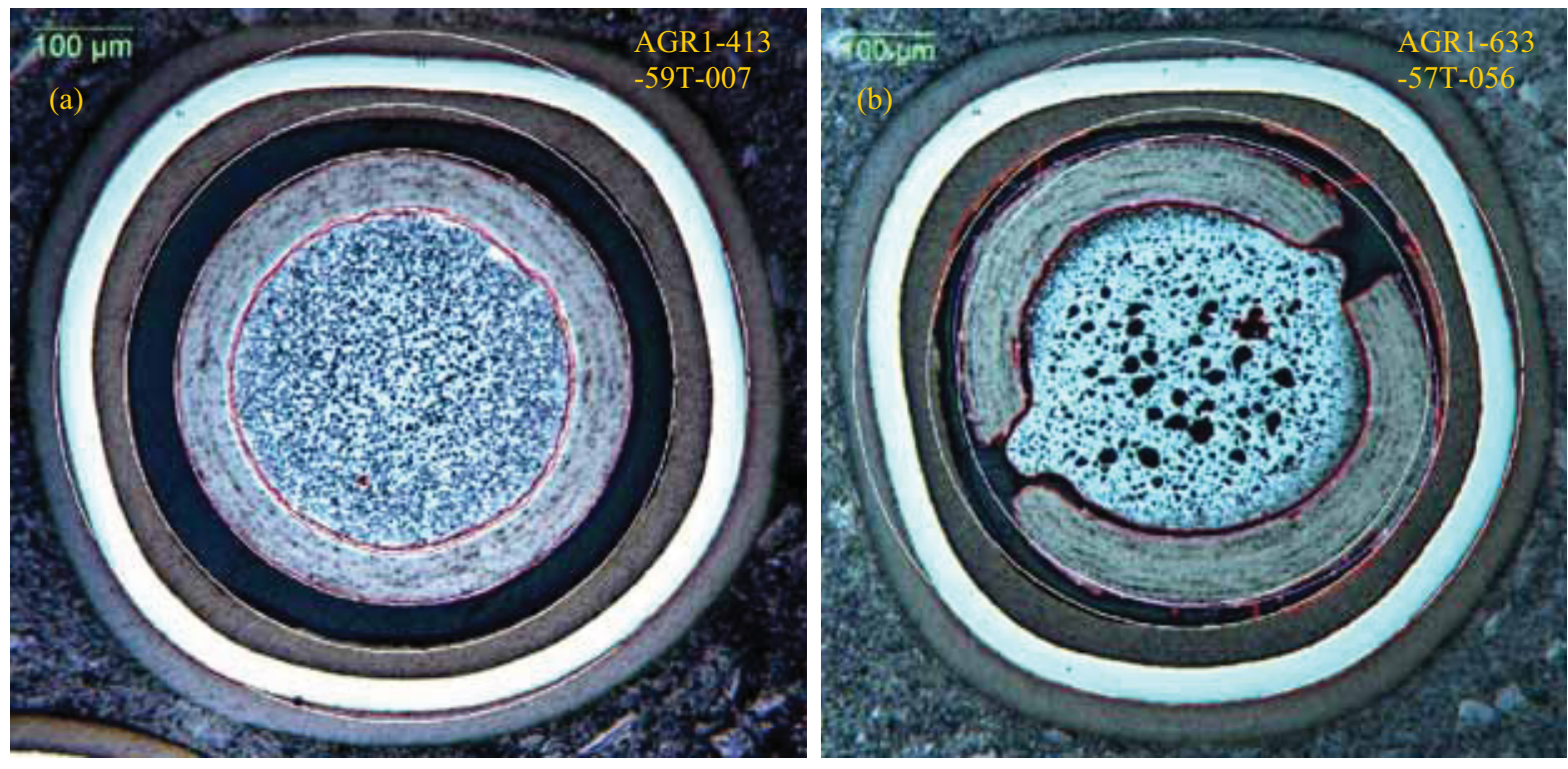

Figure 38. Circles (white), polygons (red), and arcs (blue) fit to particles with (a) and without (b) a fractured buffer for dimensional measurements. 
Because of their rarity, only five Type B particles were measured: four Type Bi and one Type Bf. Measurements on the Type Bf particle were essentially indistinguishable from the values on Type Bi particles, so all Type B measurements could be grouped together. Type Af Particle AGR1-213-136T-059 (shown in Figure 30) passed the screening for midplane proximity but was not measured because of the nonrepresentative amount of kernel swelling and near-total absence of buffer at the sectioned plane.

Kernel surface areas (cross-sectional area in the polished plane) are presented by compact in Figure 39. The distributions are uniformly wide for all three compacts, which disguises any real variation among these compacts because of burnup or irradiation temperature. Nevertheless, the vast majority indicate swelling relative to the as-fabricated average kernel area of 0.096 at midplane. Lower area fractions (superficially suggesting shrinkage) probably result from kernel centers far outside the sectioned plane. Besides the variability in kernel center offsets, other factors contributing to the wide distributions in Figure 39 are: (1) particles and kernels had substantial diameter ranges before irradiation, and (2) kernel growth apparently varied depending on the amount and duration of constraint imposed by the buffer layer.

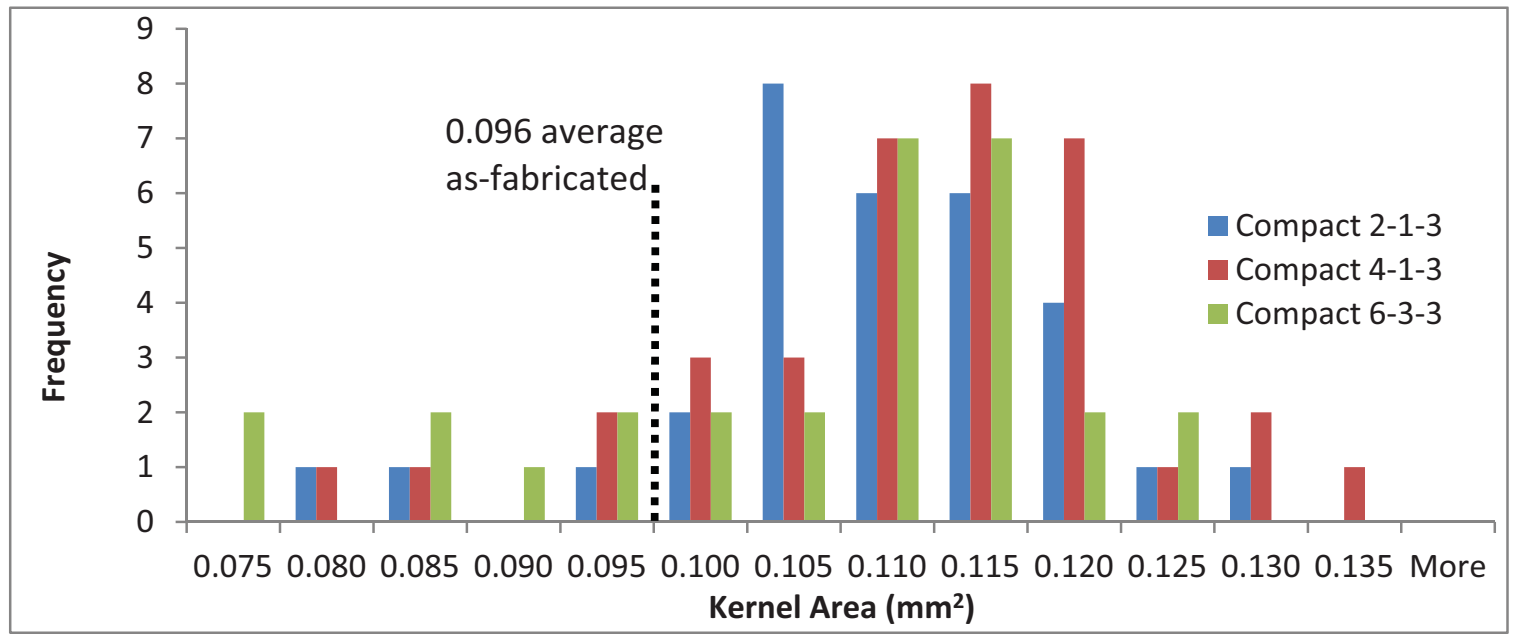

Figure 39. Histogram of 95 irradiated cross-sectional kernel areas.

Influence of buffer-imposed constraint on kernel growth is explored in Figure 40. Kernel growth in Type Ai and Type ABi particles was always constrained by an intact buffer that densified radially inward. Somewhat less constraint may have been imposed on Type Af and Type ABf particles after buffer fracture, especially where kernels swelled into open buffer fractures. However, less post-fracture constraint on Type Af/ABf kernel growth is not consistently reflected as larger areas in Figure 40, which could be explained by some buffers fracturing late during the AGR-1 irradiation. Another interpretation is that some buffer fractures may not have released all tension around kernel peripheries. That is, some fractures separated buffers into distinct pieces while other buffers merely split partway around (maintaining some constraint on kernel growth). In any case, although buffer fracturing induced local kernel growth into fracture cavities, the overall influence of buffer fracturing on kernel swelling evidently was minor. Stronger evidence of more kernel swelling is found in Figure 40 for Type B particles, where the buffers stayed bonded to IPyC layers and densified radially outward, imposing less constraint on kernel growth throughout the AGR-1 irradiation compared to Type A or Type AB particles. 


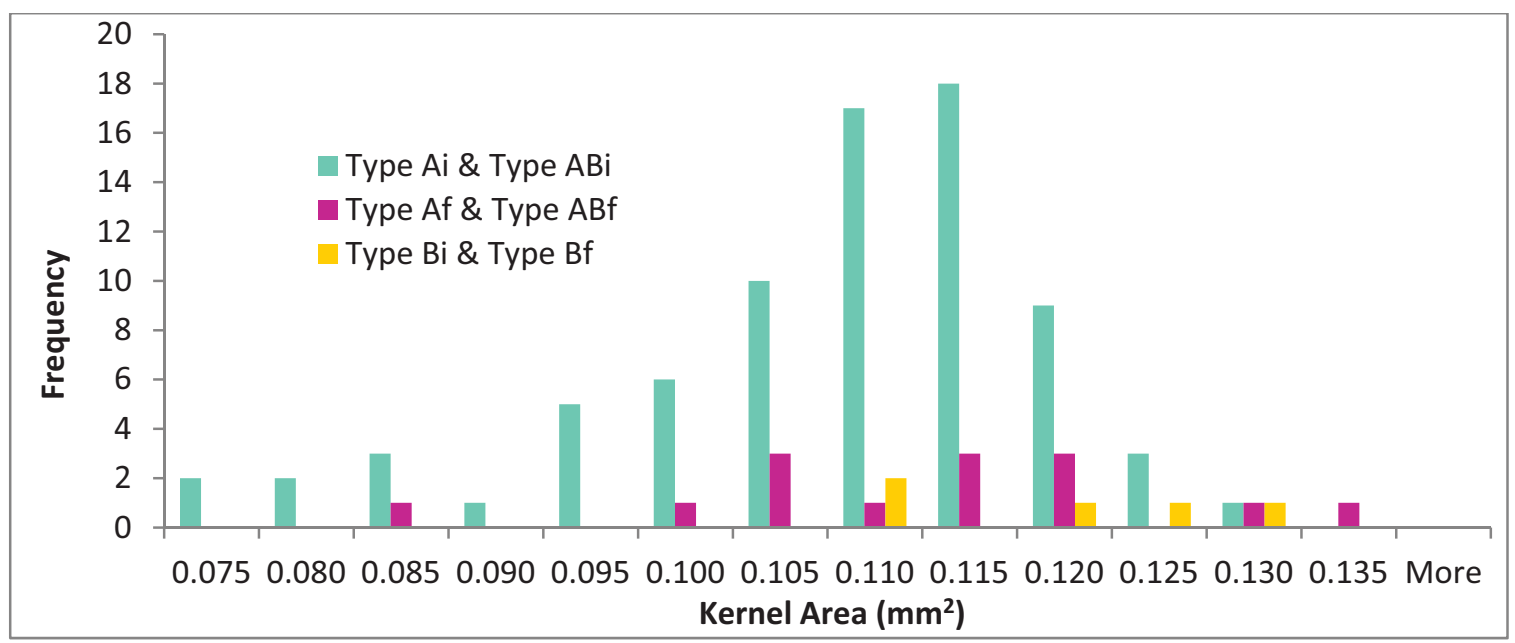

Figure 40. Kernel areas grouped according to buffer-imposed constraint on kernel growth.

A similar pattern of buffer constraint influence is displayed in Figure 41 for kernel pore area, which was measured as a percentage of the kernel area inside the low porosity kernel exterior. (Figure 41 values should not be interpreted as volumetric porosity.) Type B particles with the least buffer-imposed constraint typically exhibit larger pore area. Some Type A and Type AB particles with fractured buffers are distinct from those with intact buffers, while many Type Af/ABf particles blend in with Type Ai/ABi particles. This inconsistent behavior for Type Af/ABf particles may again infer that buffer fractures happened at different times during the AGR-1 experiment. Another factor may have been differences in post-fracture kernel constraint depending on the circumferential extent of the buffer fractures (complete separation into distinct pieces versus partial splits). Incidentally, boundaries of pores were blurred by the relatively coarse pixel size used in the analyzed images. Pixels straddling pore boundaries were not counted as porosity, which reduced measured pore areas and shifted histogram columns in Figure 41 to the left. (Higher resolution images of kernels are recommended for porosity measurements in the future.) This problem had disproportionate influence on areas of smaller pores, so particles with more small pores were shifted farther to the left in the histogram.

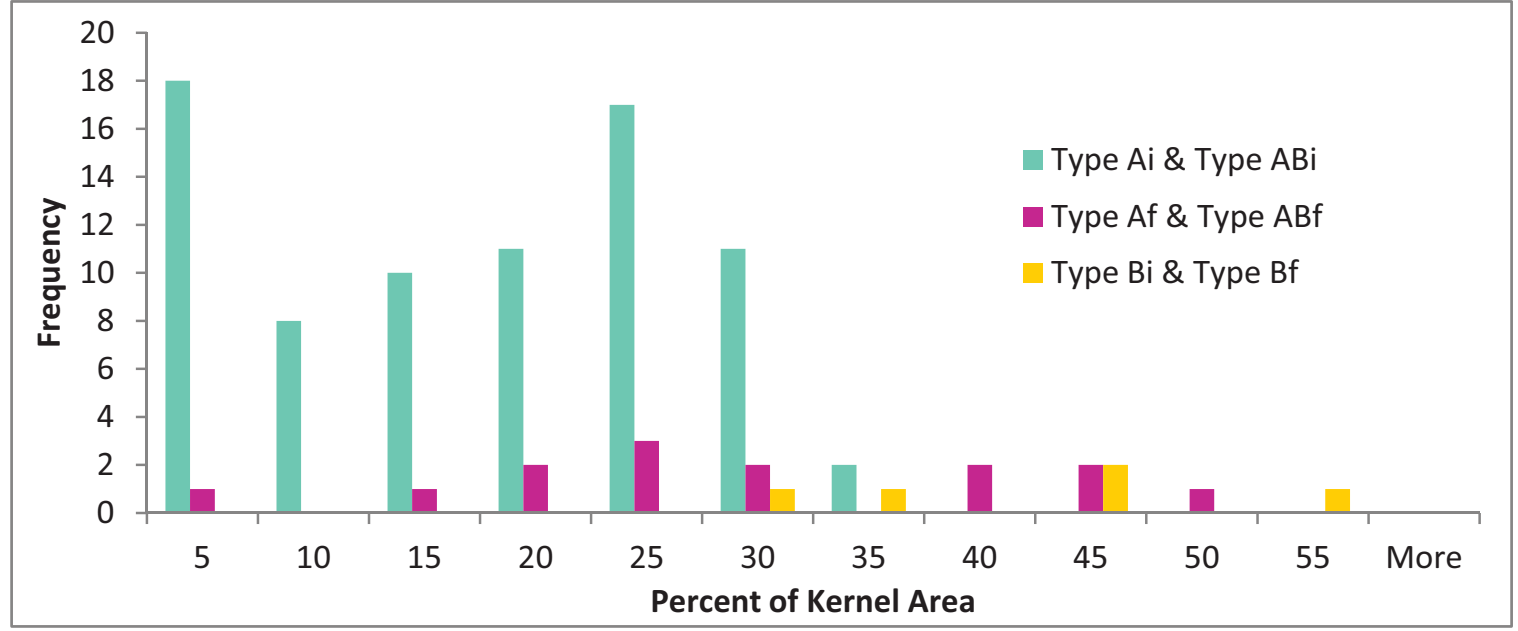

Figure 41. Pore area percentages in kernels according to buffer-imposed constraint on kernel growth.

Figure 42 reveals lower pore area percentages in most Compact 4-1-3 kernels than in kernels in the other two compacts. (This appears to be primarily because of the smaller pores in Compact 4-1-3 kernels 
whose areas were poorly detected with the intermediate resolution pixel size, as discussed for Figure 41, although total pore areas in Compact 4-1-3 kernels could possibly be smaller as well.) As shown in Table 1, Compact 4-1-3 experienced relatively high time-average volume-average temperature $\left(1075^{\circ} \mathrm{C}\right)$ and time-average maximum temperature $\left(1184^{\circ} \mathrm{C}\right)$, but Compact 6-3-3 average temperatures were similar $\left(1106^{\circ} \mathrm{C}, 1174^{\circ} \mathrm{C}\right)$ while Compact $2-1-3$ average temperatures were much lower $\left(988^{\circ} \mathrm{C}, 1097^{\circ} \mathrm{C}\right)$. Therefore, the lower porosity in Compact 4-1-3 kernels indicated in Figure 42 does not appear to have been a temperature effect. Compact 4-1-3 had higher burnup than the other two compacts according to Table 1, but higher burnup and more fission gas generation might have been expected to create more porosity. Consequently, a satisfactory explanation for the lower indicated porosity in Compact 4-1-3 is currently lacking.

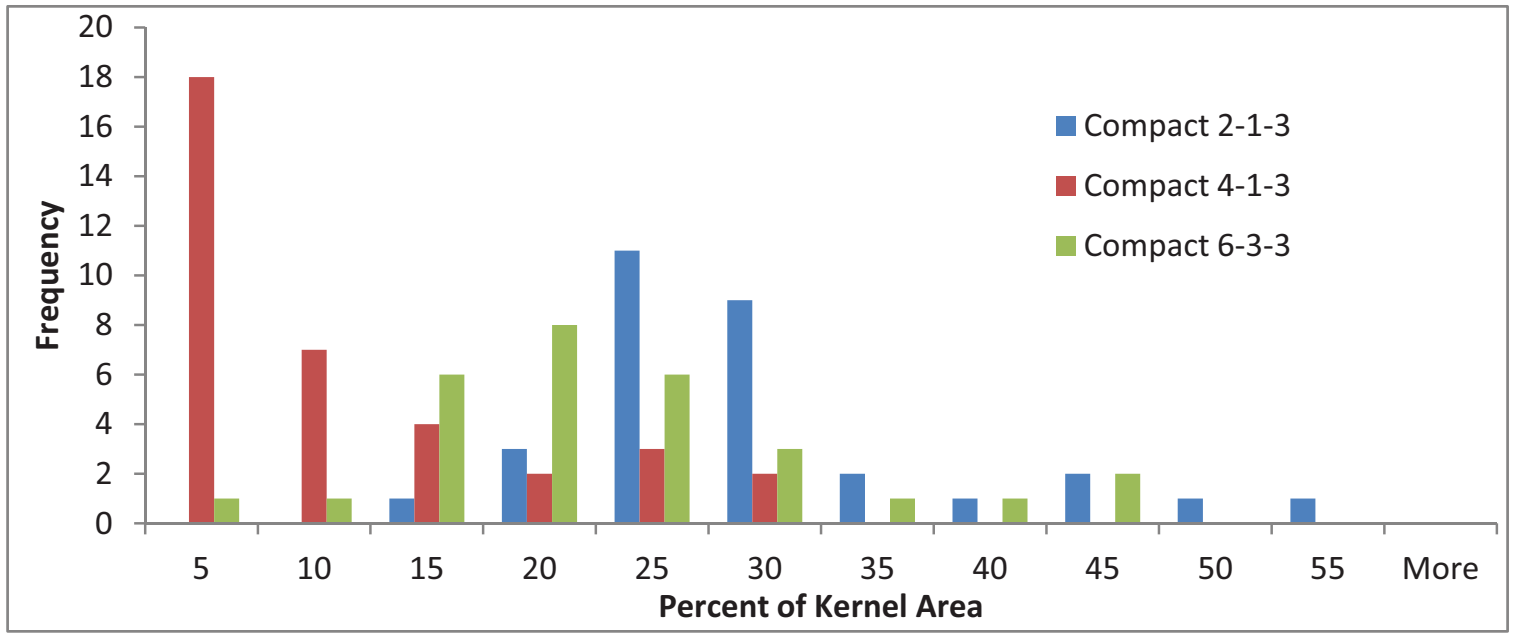

Figure 42. Kernel pore area percentages grouped by compact.

As discussed in Section 3.4.2, conspicuously larger pores were observed in some kernels. To look for trends in this regard, the diameter of the largest pore in each kernel was determined as a measure of pore enlargement. The largest pore often had an irregular outline from interconnection of several pores, so the area from a polygon trace was converted into an equivalent circular diameter. Pore enlargement among the three compacts is compared in Figure 43 for each of the 95 measured particles. Appreciably more pore enlargement is indicated in Figure 43 for pores in Compact 6-3-3 kernels, despite the similarly high average temperatures in Compact 4-1-3 given in Table 1. Pore enlargement appears very similar in Compact 2-1-3 and Compact 4-1-3, despite the appreciable difference in their irradiation temperatures. Thus differences in enlargement do not seem to correlate with differences in irradiation temperature. Similarly, there seems to be no relation between the neutron exposure values in Table 1 and the variations among compacts in Figure 43. 


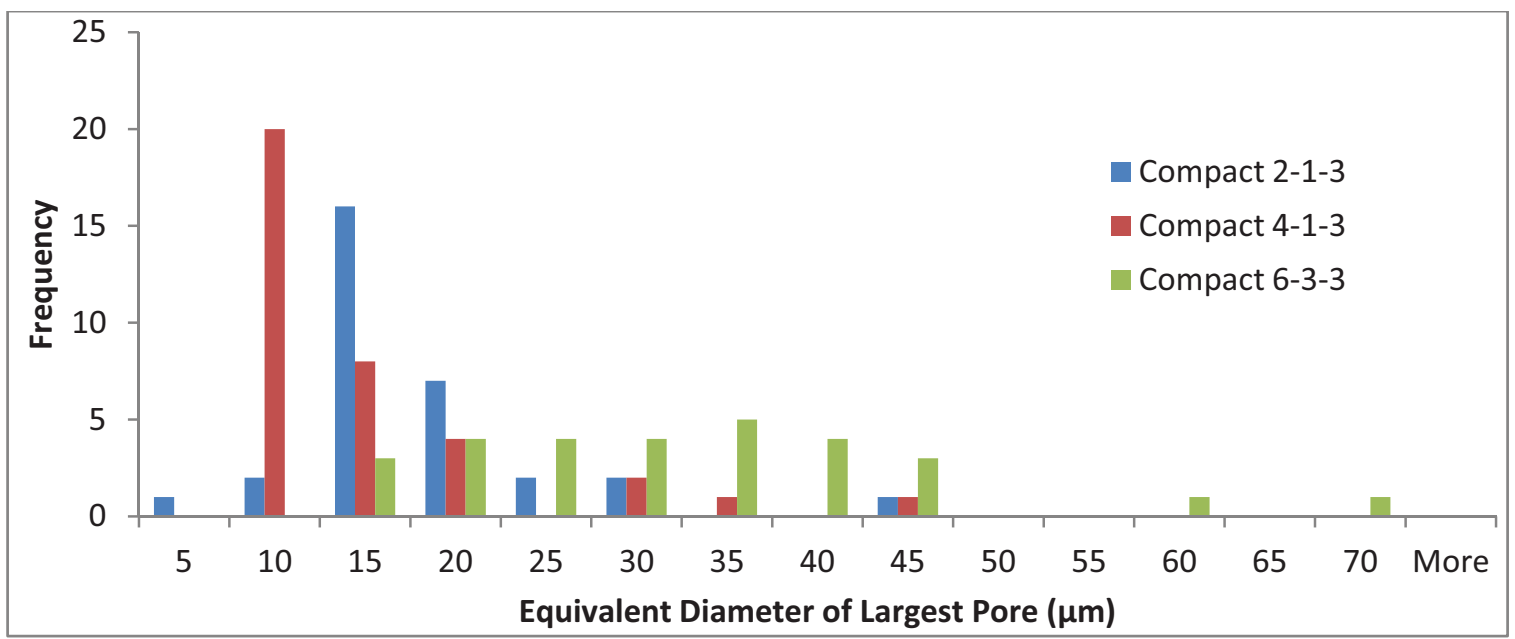

Figure 43. Size of largest pore in each kernel grouped by compact.

The same buffer constraint-based trend seen for kernel area increase in Figure 40 and kernel pore area in Figure 41 is found for pore enlargement in Figure 44. Type B particles with outward buffer densification (least constraint on kernel growth) typically show larger diameters for the biggest pore in each kernel. Significantly less enlargement is indicated for Type Ai and Type ABi particles where inward buffer densification always constrained kernel growth. Intermediate maximum pore diameters are seen for Type Af and Type ABf particles, where buffers densified inward until they fractured.

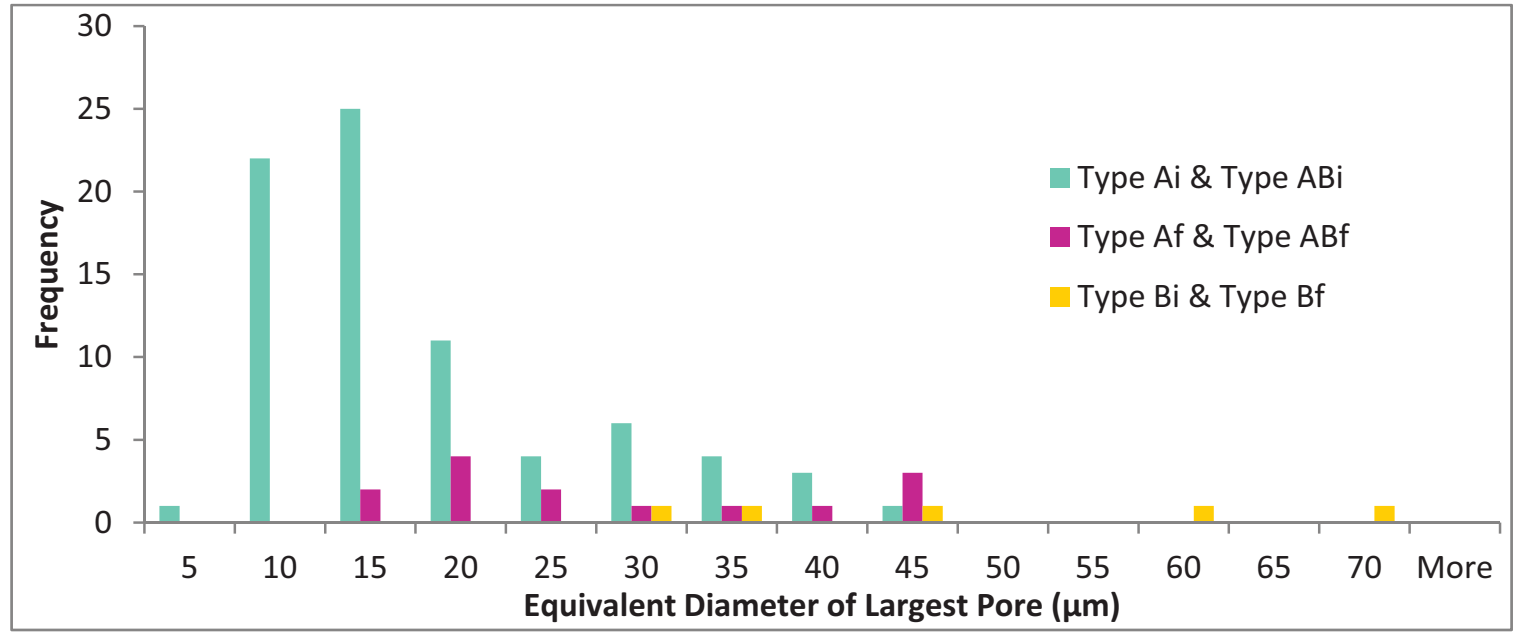

Figure 44. Largest kernel pore sizes grouped according to buffer-imposed constraint on kernel growth.

No obvious difference among compacts can be found in the buffer areas displayed in Figure 45. Variations in as-fabricated kernel size, buffer thicknesses, and post-irradiation buffer location within the particle (nonconcentricity from buffer-IPyC gap formation) contribute to the wide distributions in Figure 45. Nevertheless, because the as-fabricated buffer had an average cross-sectional area of $0.149 \mathrm{~mm}^{2}$, buffers in all measured particles exhibited evidence of densification. 


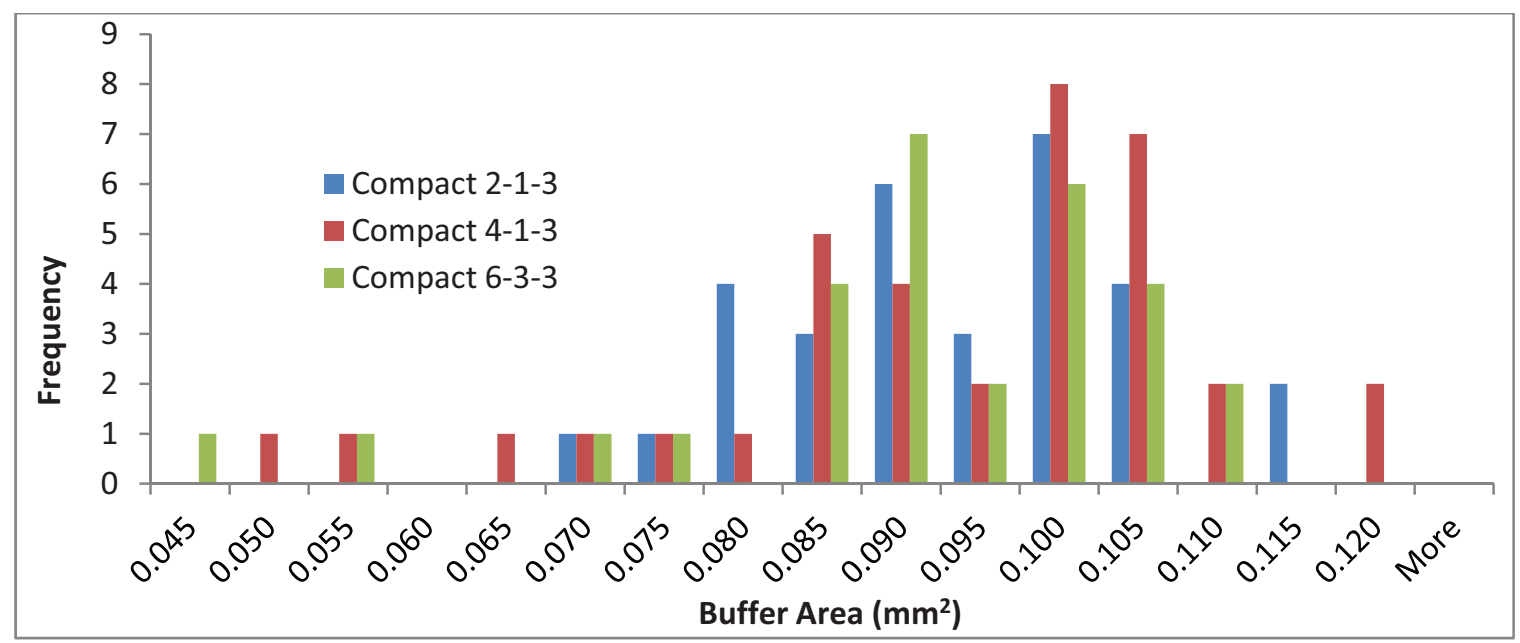

Figure 45. Buffer cross-sectional areas grouped by compact.

Buffer areas are presented in Figure 46 according to the same groups of particle types as in Figures 40, 41, and 44. However, the legend in Figure 46 reflects buffer bonding and fracturing behavior rather than buffer-imposed constraint on kernel growth. Buffer areas apparently shrank by approximately the same amounts independent of buffer fracturing and whether buffers debonded from the IPyC layer or remained fully bonded to it.

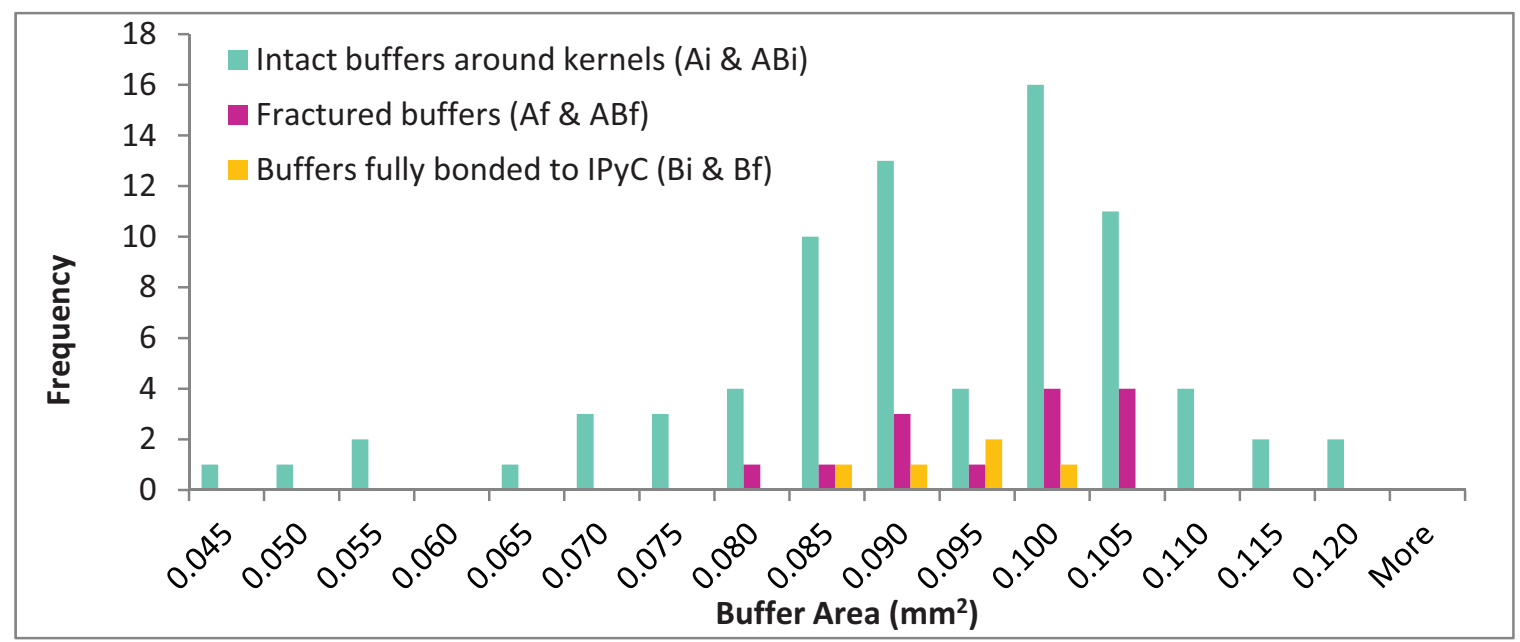

Figure 46. Buffer cross-sectional areas grouped according to buffer bonding and fracturing behavior.

Average buffer thickness results are presented by compact in Figure 47. Figure 47 again shows wide distributions because of deviations from concentricity and variation in as-fabricated dimensions. There is no obvious difference among the compacts except for the unexplained narrower range for Compact 2-1-3, which may be circumstantial. All 95 AGR-1 particles measured show a significant thickness decrease compared to the as-fabricated average buffer thickness for these compacts of $103.5 \mu \mathrm{m}$. 


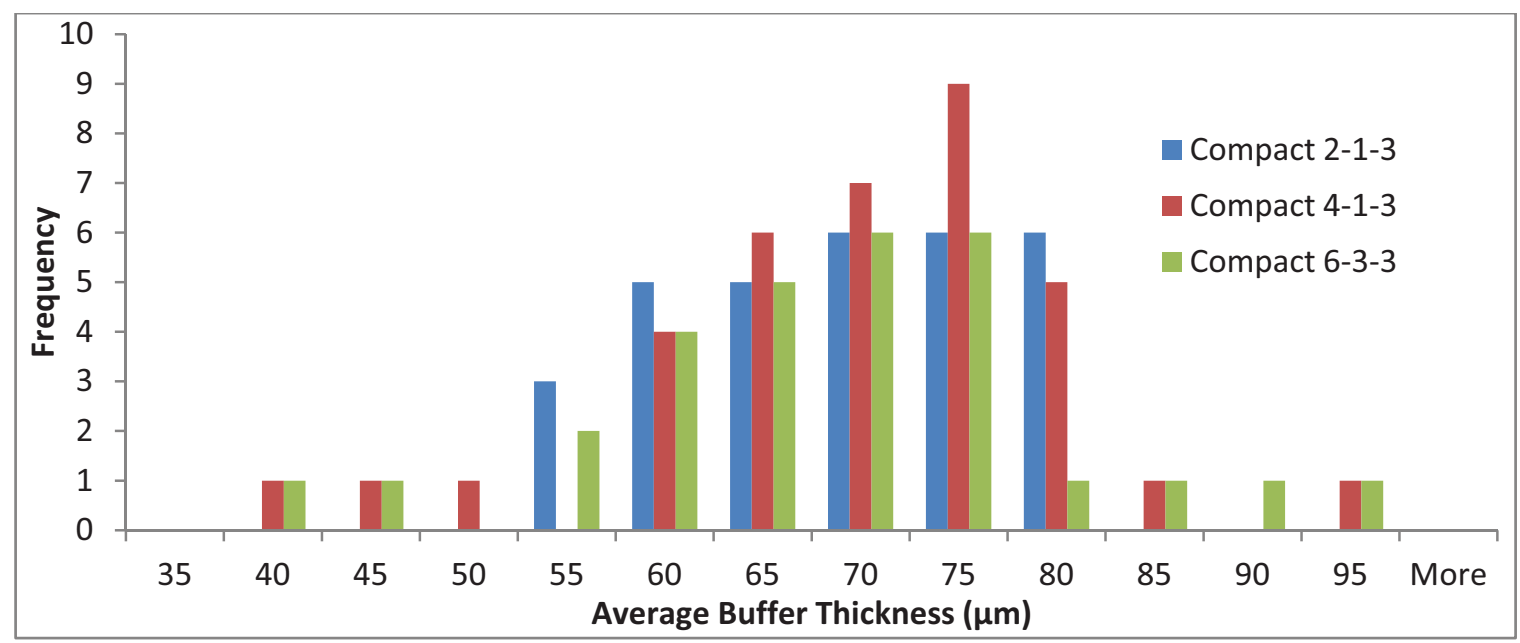

Figure 47. Buffer thicknesses grouped by compact.

Average buffer thicknesses are shown in Figure 48 after being arranged by buffer behavior during irradiation. According to Figure 48, buffer thickness apparently shrank by approximately the same amount with radially inward densification (Type A and Type AB particles with intact buffers) as with radially outward densification (Type B particles with full buffer-IPyC bonding). Buffer fracturing also had no obvious impact on thickness reduction.

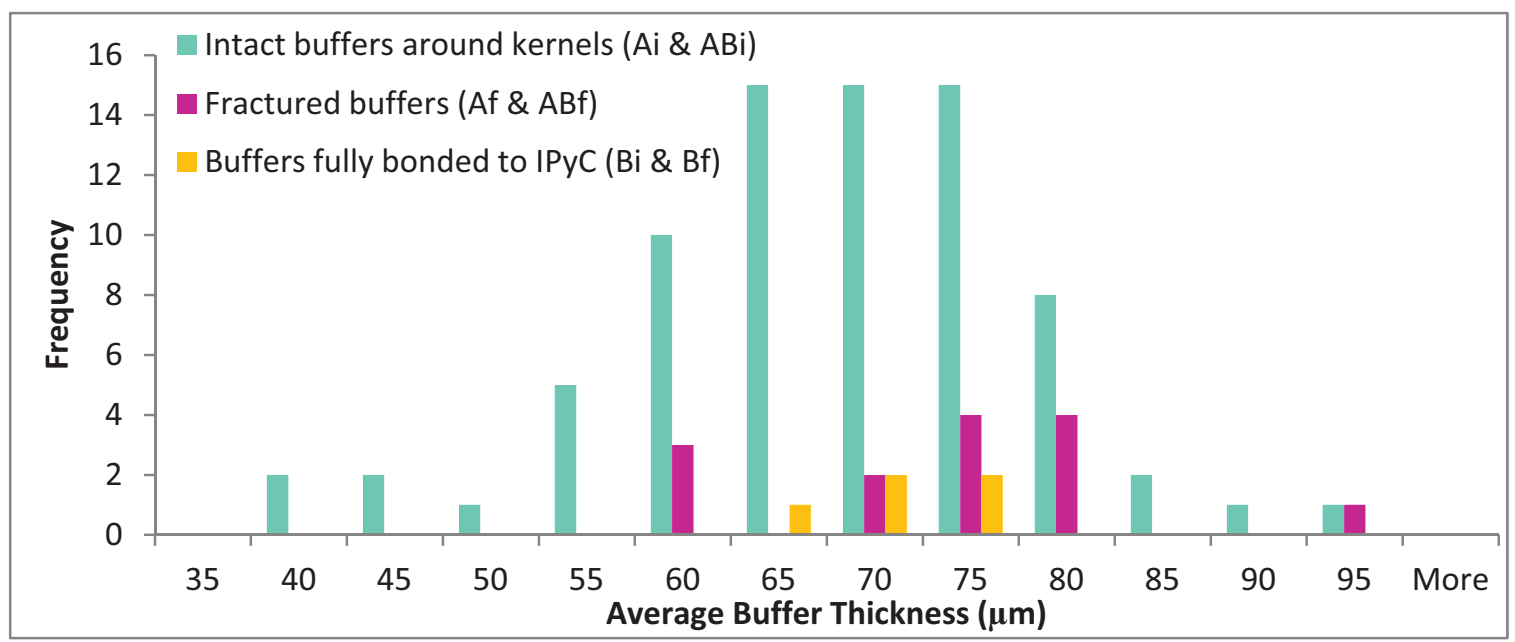

Figure 48. Buffer thicknesses grouped by buffer bonding and fracturing behavior.

Because of fundamental geometrical differences between Type A particles (inward buffer densification around a swelling kernel) and Type B particles (outward buffer densification away from a swelling kernel), buffer thickness and buffer area measured from the near-midplane cross sections are poor metrics for estimating and comparing volumetric densification of the buffers. Moreover, similar buffer areas and thicknesses in the two particle types (as indicated in Figures 46 and 48) may actually indicate that volumetric densification was significantly different between the two types. Additional methods that may help overcome the inherent limitations of the 2-D, single-plane analysis are discussed in the Recommendations.

Average buffer-IPyC gap thicknesses are arranged according to buffer behavior in Figure 49. The average gap thickness for all measured particles was approximately $24 \mu \mathrm{m}$, which included Type B 
particles where buffers stayed attached to IPyC interiors around the entire interface and where no gaps existed. The average gap thickness for Type Ai and Type ABi particles was approximately $27 \mu \mathrm{m}$. Some gaps with fractured buffers were smaller, which reduced the average for Type Af and Type ABf particles to approximately $14 \mu \mathrm{m}$. By inspection, the smaller Type Af and Type ABf gaps coincide with wide cavities between buffer pieces, where kernel swelling apparently pushed the buffer pieces apart. No significant buffer-IPyC gap variations can be seen among compacts in Figure 50.

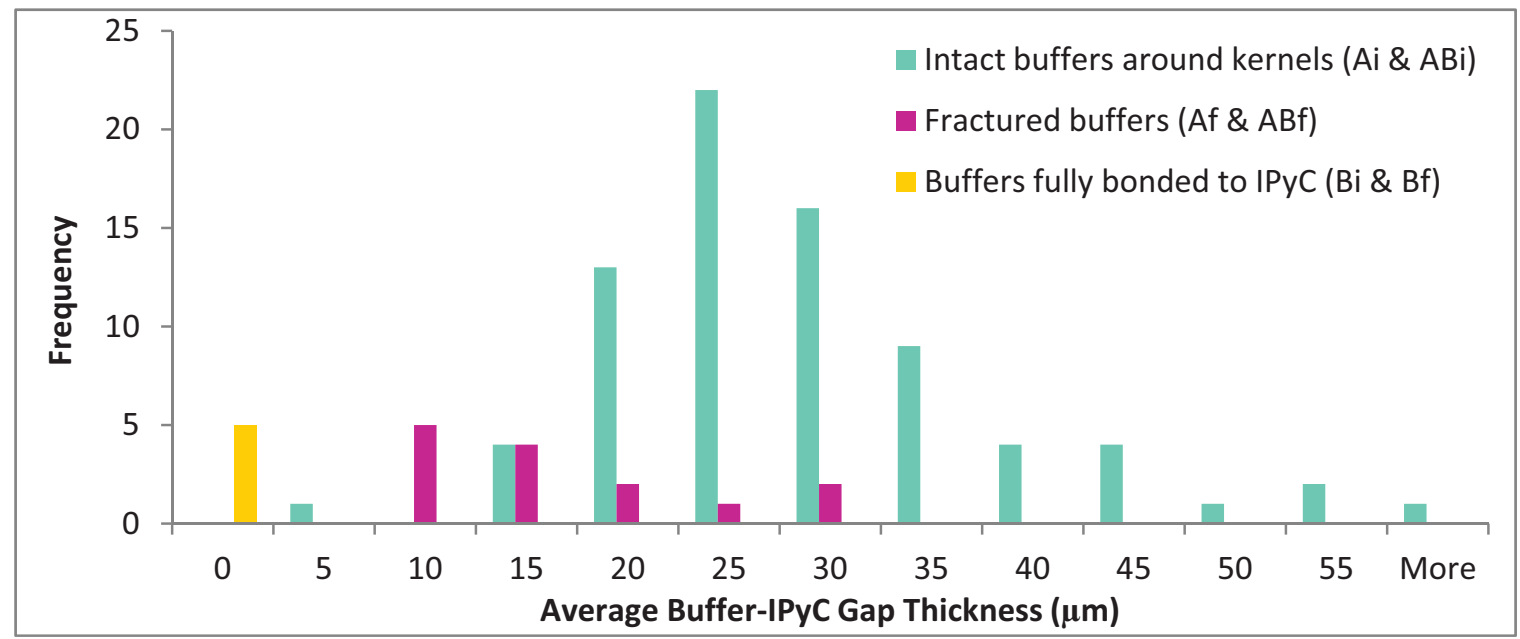

Figure 49. Average buffer-IPyC gaps grouped by buffer densification and fracturing behavior.

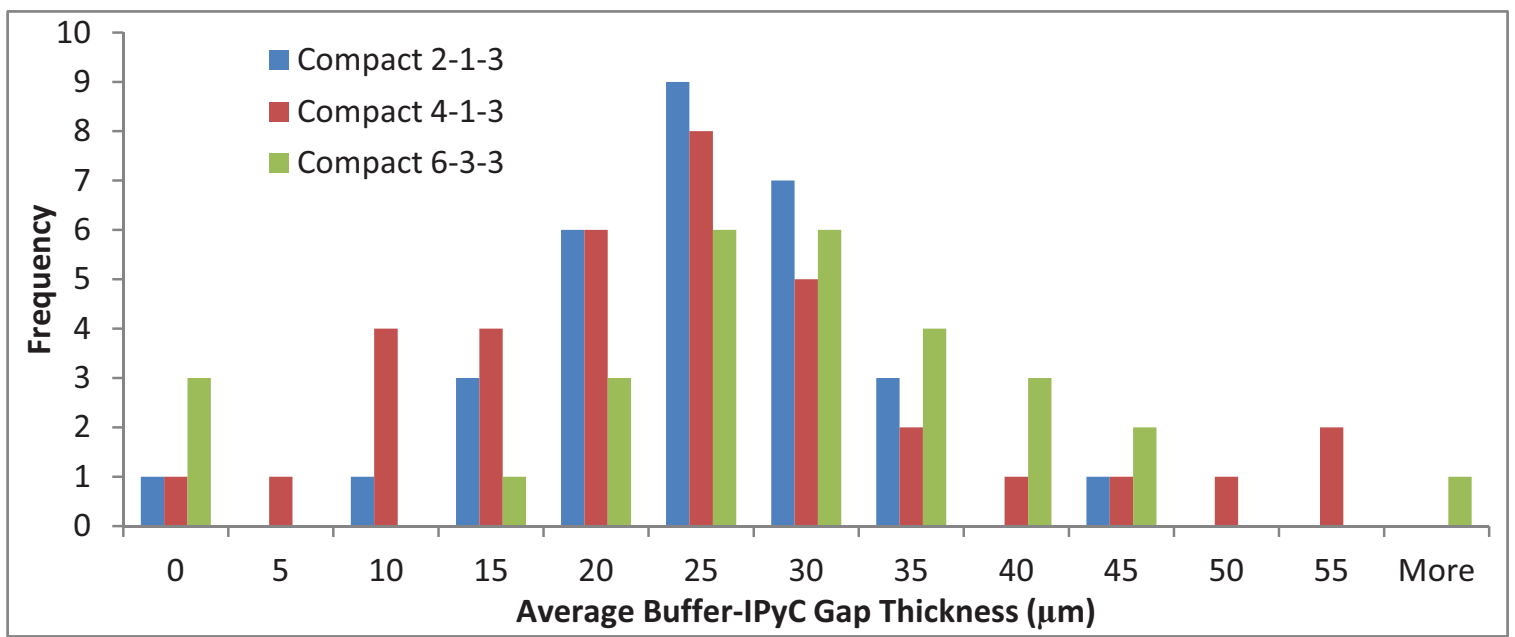

Figure 50. Average buffer-IPyC gaps grouped by compact.

\section{RECOMMENDATIONS}

Measurements of kernel porosity were made on intermediate magnification images of entire particles, but this did not allow smaller pores to be resolved well enough for proper quantification of their areas. Substantial improvement likely could be attained by taking high resolution images of the kernels. Better resolution might also help to include the entire kernel in the pore area measurements. The low porosity exterior had to be manually and subjectively excluded from kernel pore area measurements in order to make all porosity measurements with a single gray level threshold. 
The extreme ranges of behavior observed among individual images exposed the limitation of 2-D cross sections for describing complicated 3-D structures. Even after screening out particles in compact cross sections that were polished far from midplane, the remaining positional uncertainty of kernel centers could induce errors exceeding 50\% in kernel volumes computed from measured kernel areas. Estimating buffer area, buffer thickness, and buffer-IPyC gap thickness is less affected by uncertainty in kernel center location, but it could still result in errors as high as $20 \%$. Estimating dimensional changes from preirradiation values is further complicated by variations in as-fabricated sizes. Two efforts have been initiated to better visualize and quantify phenomena such as kernel swelling, buffer fracturing, and buffer densification. X-ray tomography is being performed on individual particles from deconsolidated AGR-1 compacts at ORNL. This will provide 3-D views of kernel and coating behavior that can be compared with existing 2-D data. Tomographic measurements on kernels and buffers should be immune to the severe positional uncertainties encountered in this investigation, and ideally they can assess asymmetrical behavior such as kernel swelling into fractured buffer cavities. Ensembles of at least 50 deconsolidated particles are also being mounted and will be ground, polished, and characterized at multiple levels with measured separations to provide more 2-D views of each particle during microscopy. The multilevel approach on deconsolidated particles will avoid the staggered particle elevations in compact cross sections and should also enable adjusting measured dimensions to better estimate those at midplane. It may also help to mitigate other geometry-related effects, such as ranges of fabrication sizes.

\section{CONCLUSIONS}

Careful sample preparation allowed microscopic examinations of 830 irradiated TRISO particles within cross sections of five AGR-1 compacts. Detailed images of so many particles supported development of a comprehensive classification system that fostered comparative insights on many aspects of internal behavior.

No significant spatial patterns were revealed within compact cross sections by mapping the endstate characteristic morphological types.

Particles were classified primarily by the extent of buffer-IPyC bonding, where debonding appeared driven by inward buffer densification. Type A particles that completely debonded along the buffer-IPyC interface (formed a continuous radial buffer-IPyC gap) were most numerous $(\sim 62 \%)$. Type B particles with fully bonded buffer and IPyC layers were rare $(<6 \%)$. Type AB particles with partial debonding were found with intermediate frequency $(\sim 33 \%)$. The relative proportions of these three basic types were similar among AGR-1 compacts and, therefore, were not strongly influenced by differences in irradiation temperature or neutron exposure.

Particles were classified secondarily by buffer integrity. Over three-fourths of the buffers remained intact. Buffer fracturing was over twice as frequent in Type AB particles with partial buffer-IPyC debonding than in Type A particles with complete debonding.

Buffer fractures in Type A particles typically had smooth contours in the radial direction. Buffer fractures in Type AB particles tended to have jagged contours (multiple fracture directions). Different buffer fracture mechanisms may have dominated in these two basic particle types.

A buffer fracture with full buffer-IPyC bonding (Type Bf particles) evidently always induced a fracture through the entire IPyC layer. Buffer fractures in Type B-like regions of Type AB particles also seemed to preferentially produce IPyC fractures.

Fractures through the entire IPyC layer were more frequent in particles with partial buffer-IPyC debonding (Type $\mathrm{AB}$ ) than in particles with complete debonding (Type A). 
In Type $\mathrm{AB}$ particles with partial buffer-IPyC bonding, IPyC fractures tended to be found where buffer-IPyC debonds terminated (where bonding appeared to be locally strong). IPyC fractures in Type $\mathrm{AB}$ particles were also much more likely with fractured buffers than with intact buffers.

By far the most fractures through the entire IPyC layer were found in the Variant 1 compact, which had the lowest IPyC density.

$\mathrm{SiC}$ layers fractured very rarely during the AGR-1 irradiation, and these tangential fractures penetrated through only a fraction of the SiC thickness. SiC fracture formation was apparently influenced by the frequency of IPyC fractures and by the extent of IPyC-SiC debonding at IPyC fracture tips. SiC fractures also appeared to be more likely when soot inclusions occurred during particle fabrication.

No fractures were observed in $\mathrm{OPyC}$ layers, even at locations where the compact matrix fractured during sample preparation.

Somewhat more kernel swelling, more total pore area, and larger kernel pores were observed in Type B particles, where less constraint was imposed on kernels by buffers that stayed bonded to IPyC interiors and densified radially outward. Significantly less pore area and smaller maximum pore diameters were measured in Type Ai and Type ABi kernels, which were always constrained by intact, inwardly densified buffers.

Despite appreciable variation in sectioned kernel positions (deviations from midplane), most AGR-1 kernels measured showed increases in cross-sectional area from the as-fabricated average. Within the broad distributions of results, kernel swelling appeared to be similar for Compacts 2-1-3, 4-1-3, and 6-3-3. Differences in estimated irradiation temperatures, estimated fast fluence, and estimated burnup among these compacts thus had no major influence on the respective amounts of kernel swelling.

Although kernels often swelled locally into buffer cavities, kernel area swelling in Type Af and Type ABf particles could not be clearly distinguished from kernel area swelling in Type Ai and Type ABi particles. Buffer fracturing evidently had more influence on where kernels swelled than on how much their areas increased.

Pore area tended to be lower in Compact 4-1-3 kernels while maximum pore diameters typically were larger in Compact 6-3-3 kernels. There was no obvious relationship to irradiation temperature and neutron exposure in either case.

All buffers measured showed substantial decreases in area and thickness from as-fabricated values, which indicates that buffers densified during the AGR-1 irradiation. The broad distributions in buffer results disguised any differences among Compacts 2-1-3, 4-1-3, and 6-3-3.

Inward buffer densification created an average buffer-IPyC gap thickness in Type Ai and Type ABi particles that was approximately twice the average gap thickness in Type Af and Type ABf particles. The

difference was evidently because of separation of buffer pieces in Type Af and Type ABf particles, which closed part of the gap after buffer fracturing.

\section{REFERENCES}

Demkowicz, P., 2010, “AGR-1 Post-Irradiation Examination Plan,” PLN-2828, Rev 1, March 2010.

Demkowicz, P., L. Cole, S. Ploger, and P. Winston, 2011, AGR-1 Irradiated Test Train Preliminary Inspection and Disassembly First Look, INL/EXT-10-20722, January 2011. 
Grover, S.B., D.A. Petti, and J.T. Maki, 2010, "Mission and Status of the First Two Next Generation Nuclear Plant Fuel Irradiation Experiments in the Advanced Test Reactor," ICONE18-30139,

Proceedings of the 18th International Conference on Nuclear Engineering, Xi'an China, May 17-21, 2010.

Grover, S.B., 2010. "Completion of the First NGNP Advanced Gas Reactor Fuel Irradiation Experiment, AGR-1, in the Advanced Test Reactor," Paper 104, Proceedings HTR-2010, Prague, Czech Republic, October 18-20, 2010.

Harp, J.M., 2011, "Analysis of Individual Compact Fission Product Inventory and Burnup for the AGR-1 TRISO Experiment Using Gamma Spectrometry,” ECAR-1682, September 2011.

Harp, J.M., and S.A.Ploger, 2011, "Examination of Graphite Fuel Compact Holders for the AGR-1 TRISO Experiment using Gamma Spectrometry," ECAR-1709, November 2011.

Hawkes, G.L., 2012, “AGR-1 Daily As-run Thermal Analyses,” ECAR-968, Rev. 2, January 25, 2012.

Hunn, J.D., A.K. Kercher, P.A. Menchhofer, and J.R. Price, 2005, Results from ORNL Characterization of Nominal $350 \mu \mathrm{m}$ NUCO Kernels from the BWXT 59344 Batch, ORNL/TM-2005/541, January 2005 .

Hunn, J.D., and R.A. Lowden, 2006-1, Data Compilation for AGR-1 Variant 1 Coated Particle Composite LEU01-47T, ORNL/TM-2006/020, April 2006.

Hunn, J.D., and R.A. Lowden, 2006-2, Data Compilation for AGR-1 Baseline Coated Particle Composite LEU01-46T, ORNL/TM-2006/019, April 2006.

Maki, J.T., 2006, AGR-1 Irradiation Experiment Test Plan, INL/EXT-05-00593, September 2006.

Miller, G.K., D.A. Petti, and J.T. Maki, 2004, "Consideration of the effects of partial debonding of the IPyC and particle asphericity on TRISO-coated fuel behavior," Journal of Nuclear Materials, Vol. 334 , pp. 79-89.

Simonds, J., 2010, "Technical Program Plan for the Next Generation Nuclear Plant/Advanced Gas Reactor Fuel Development and Qualification Program," PLN-3636, September 2010.

Sterbentz, J.W., 2011, "JMOCUP As-run Daily Depletion Calculation for the AGR-1 Experiment in ATR B-10 Position,” ECAR-958, Rev. 1, August 11, 2011. 


\section{Appendix A}

\section{AGR-1 Compact Cross Sections}

Shown below are montages of cross sections from Compacts 2-1-3, 3-3-3, 4-1-3, 5-1-2, and 6-3-3. These figures (Figures A-1 through A-28) are generally presented as pairs where the first gives the identification numbers assigned to each particle with some exposed kernel (except as noted below) and the second figure provides distributions of particle types according to the classification scheme in Figure 11 of the main text. The second figure includes a tally of the types present with percentages.

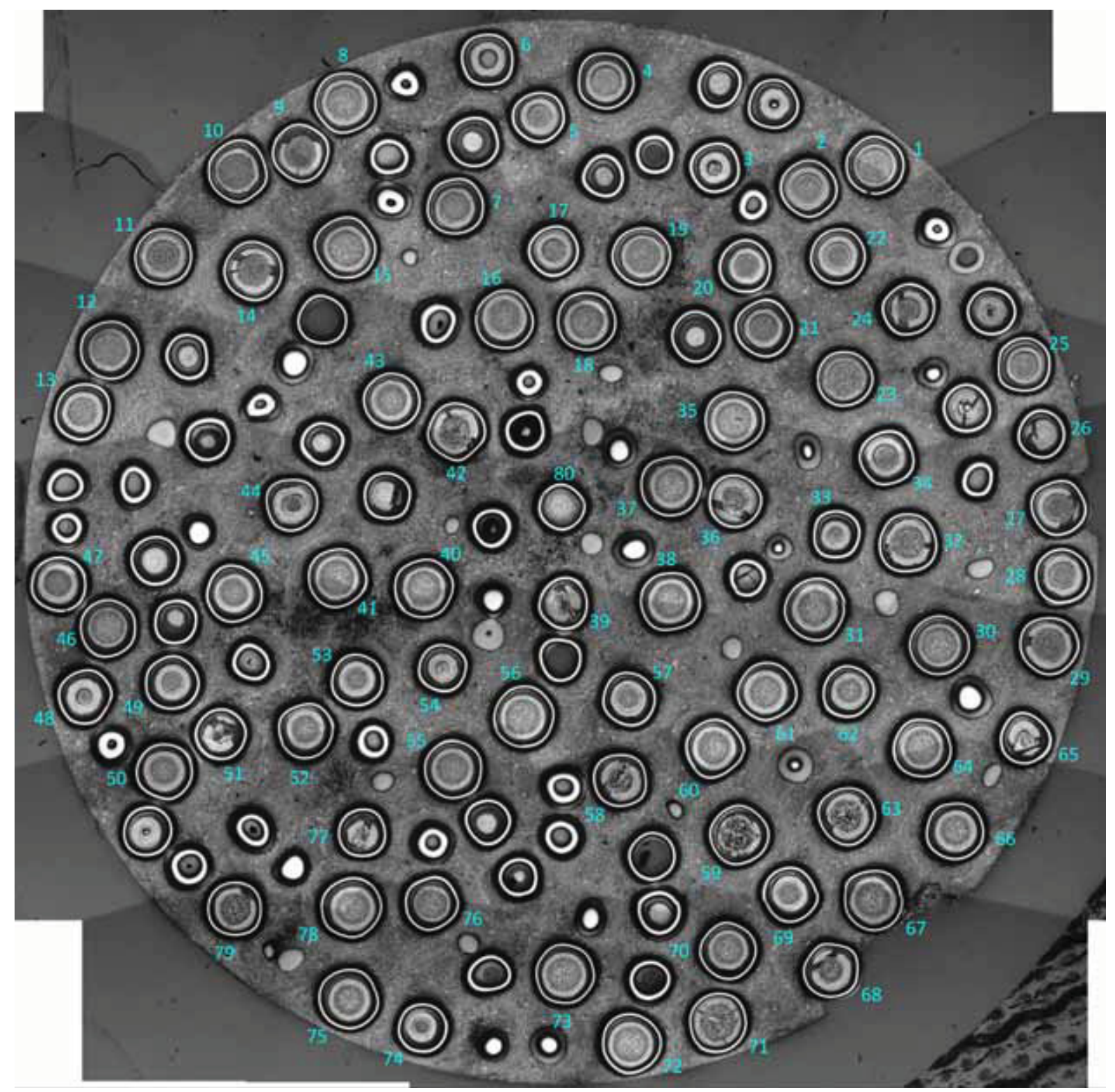

Figure A-1. Transverse cross section through middle of Compact 2-1-3 (Mount 136T) with particle identifiers.

Particle 80 had no exposed kernel but contained SiC cracks and a possible soot inclusion. 


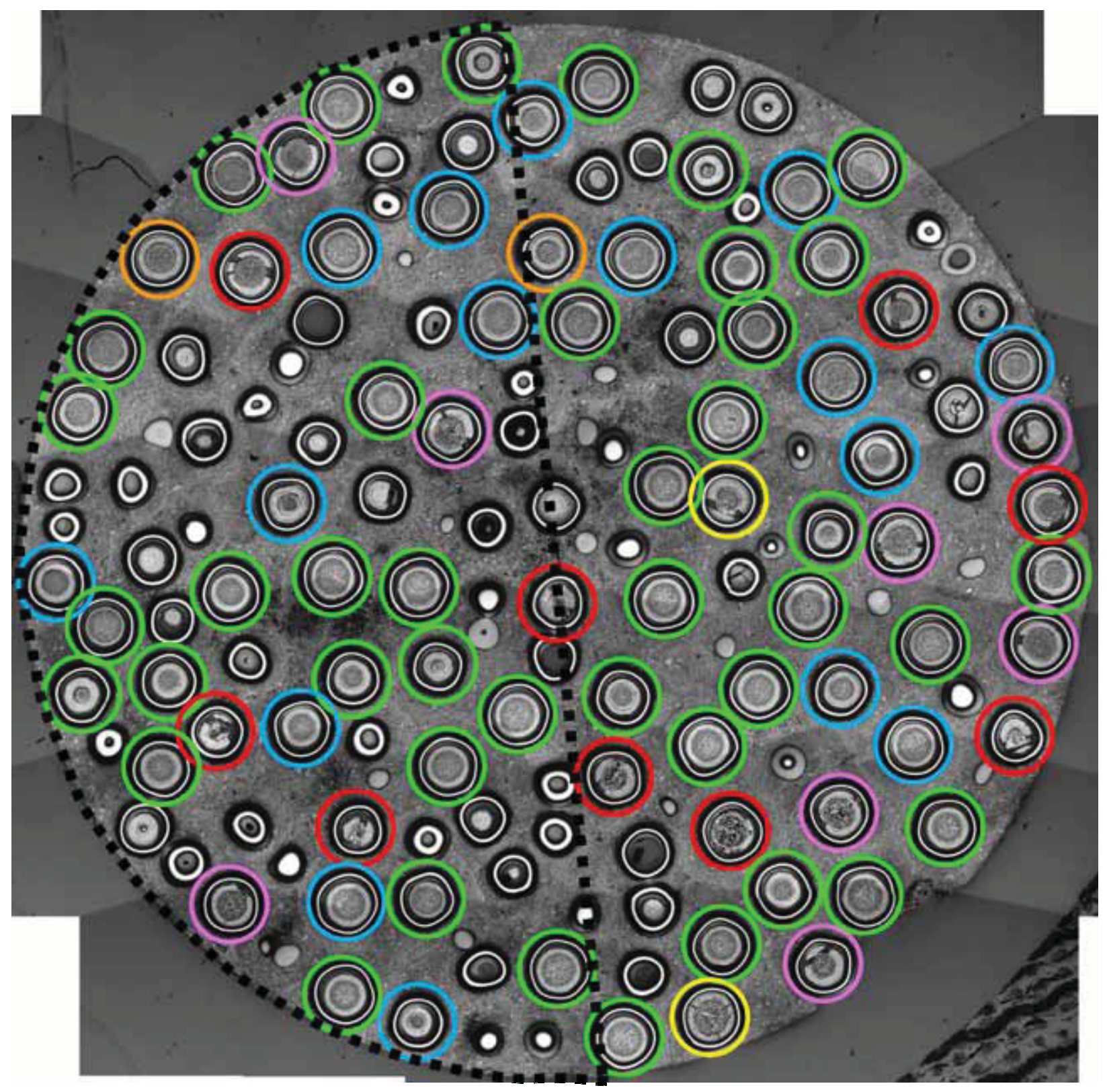

Figure A-2. Spatial distribution of particle morphologies across Compact 2-1-3 (Mount 136T).

The broken outline in Figure A-2 indicates the approximate position of the accompanying longitudinal sections (Mounts 137T and 138T in Figures A3 through A6). A total of 79 intact particles with exposed kernel were characterized as follows:

- 42 Type Ai particles (green circles), 53.2\%

- 9 Type Af particles (red circles), 11.4\%

- $\quad 16$ Type ABi particles (blue circles), 20.3\%

- 8 Type ABf particles (magenta circles), $10.1 \%$

- 2 Type Bi particles (orange circles), 2.5\%

- 2 Type Bf particles (yellow circles), $2.5 \%$. 


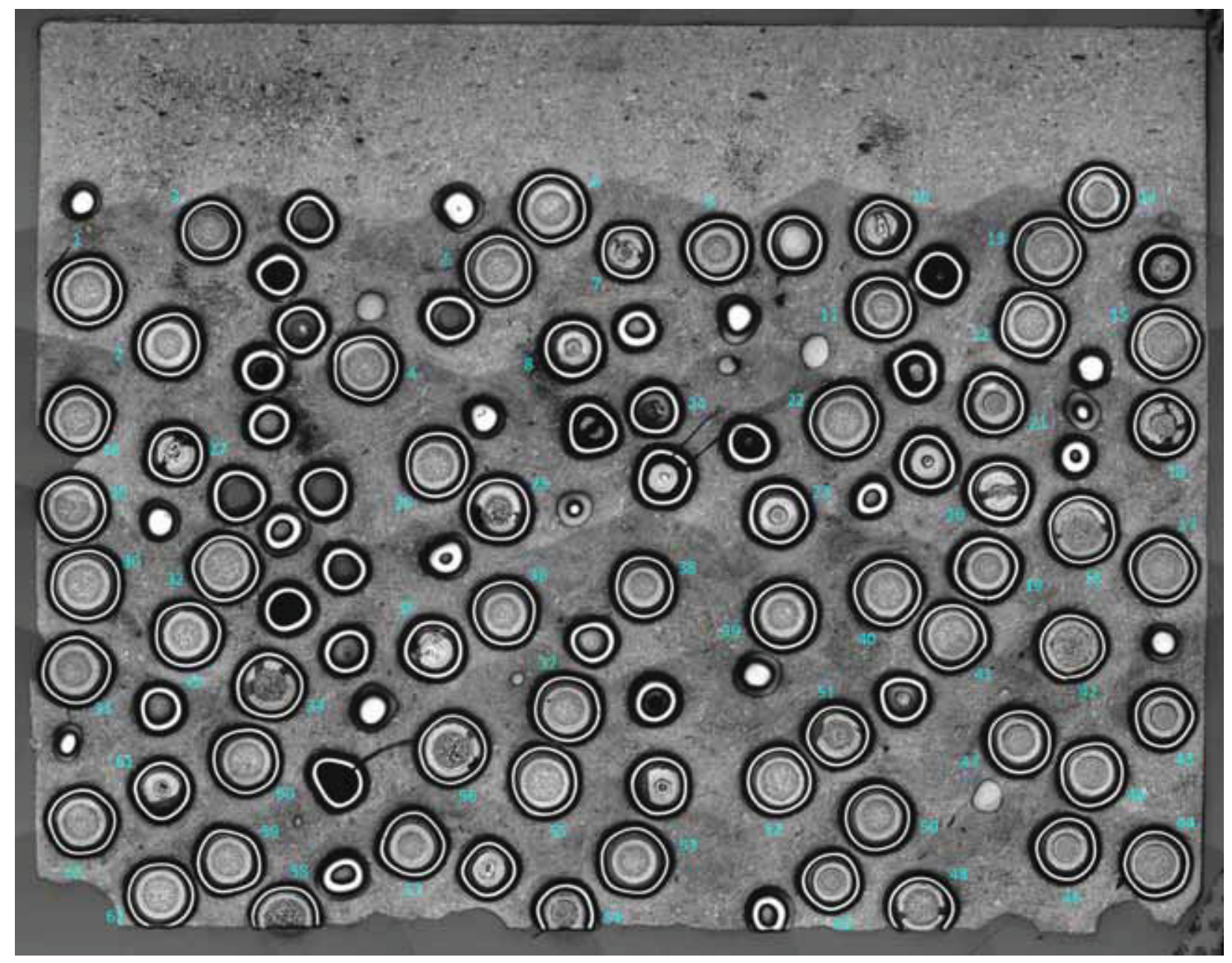

Figure A-3. Longitudinal cross section through top portion of Compact 2-1-3 (Mount 137T) with particle identifiers. 


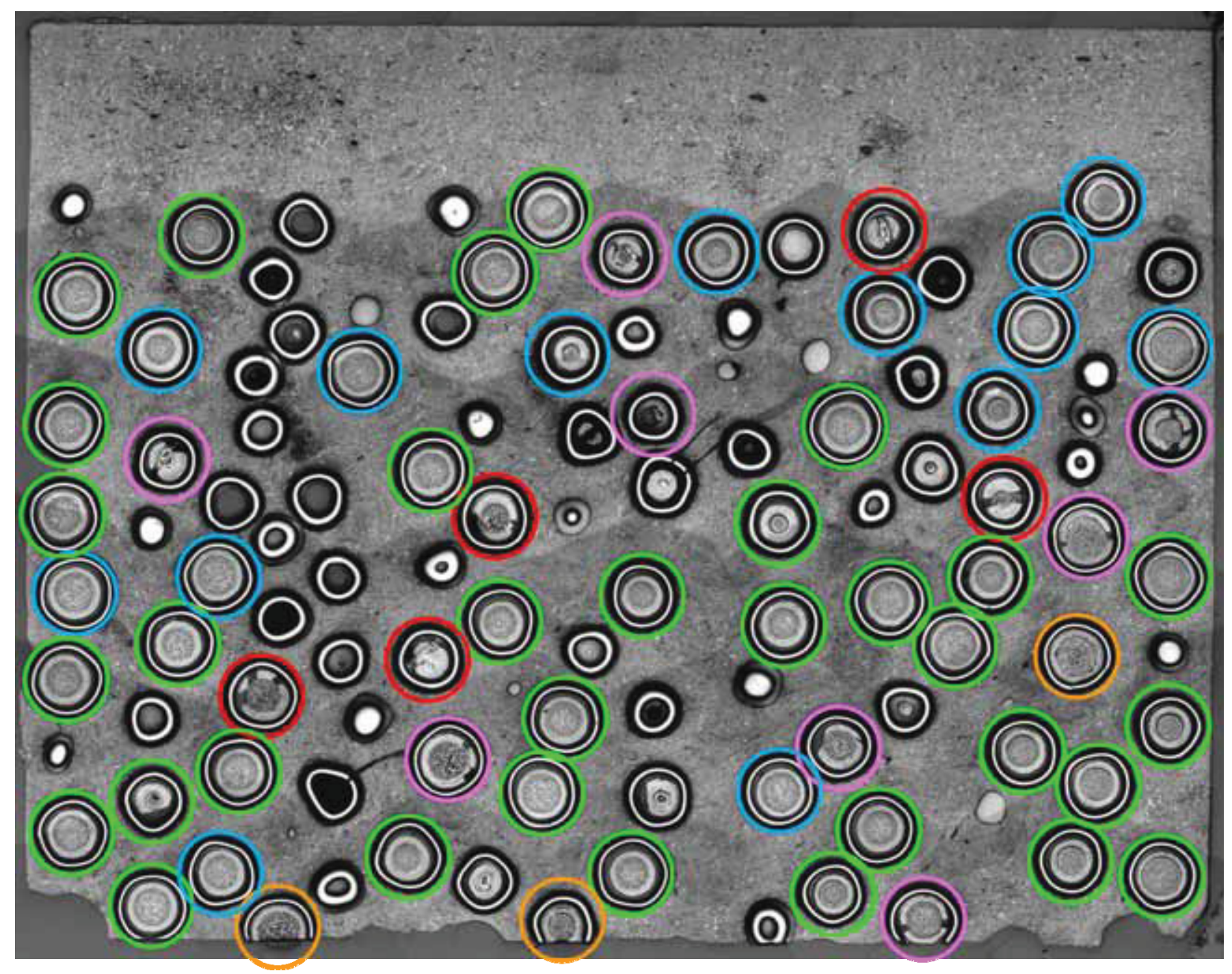

Figure A-4. Spatial distribution of particle morphologies across Compact 2-1-3 (Mount 137T).

A total of 63 intact particles with exposed kernel were characterizedas follows:

- 33 Type Ai particles (green circles), 52.4\%

- 5 Type Af particles (red circles), 7.9\%

- $\quad 14$ Type ABi particles (blue circles), 22.2\%

- 8 Type ABf particles (magenta circles), $12.7 \%$

- 3 Type Bi particles (orange circles), $4.8 \%$

- 0 Type Bf particles (yellow circles), $0.0 \%$. 


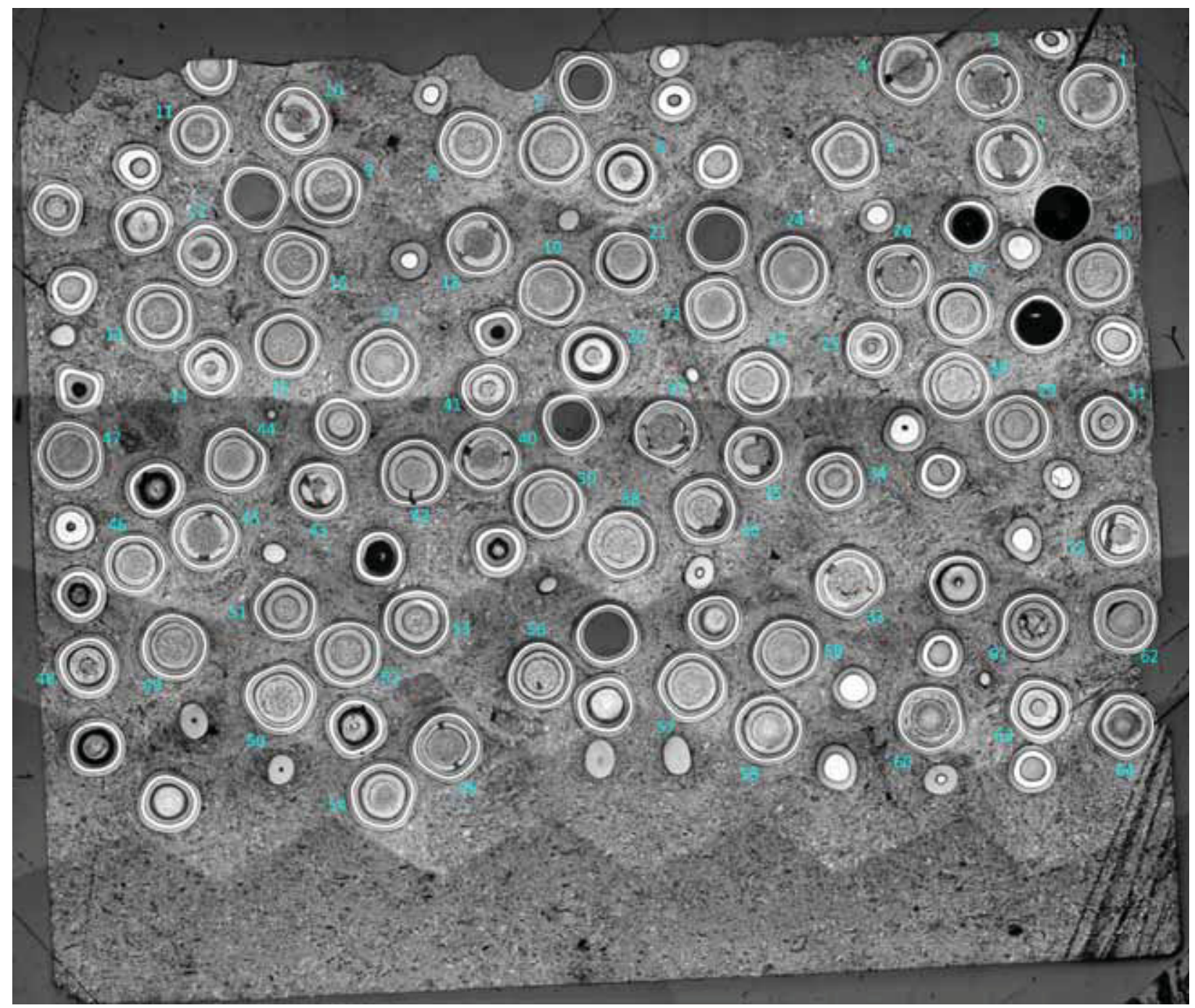

Figure A-5. Longitudinal cross section through bottom part of Compact 2-1-3 (Mount 138T) with particle identifiers. 


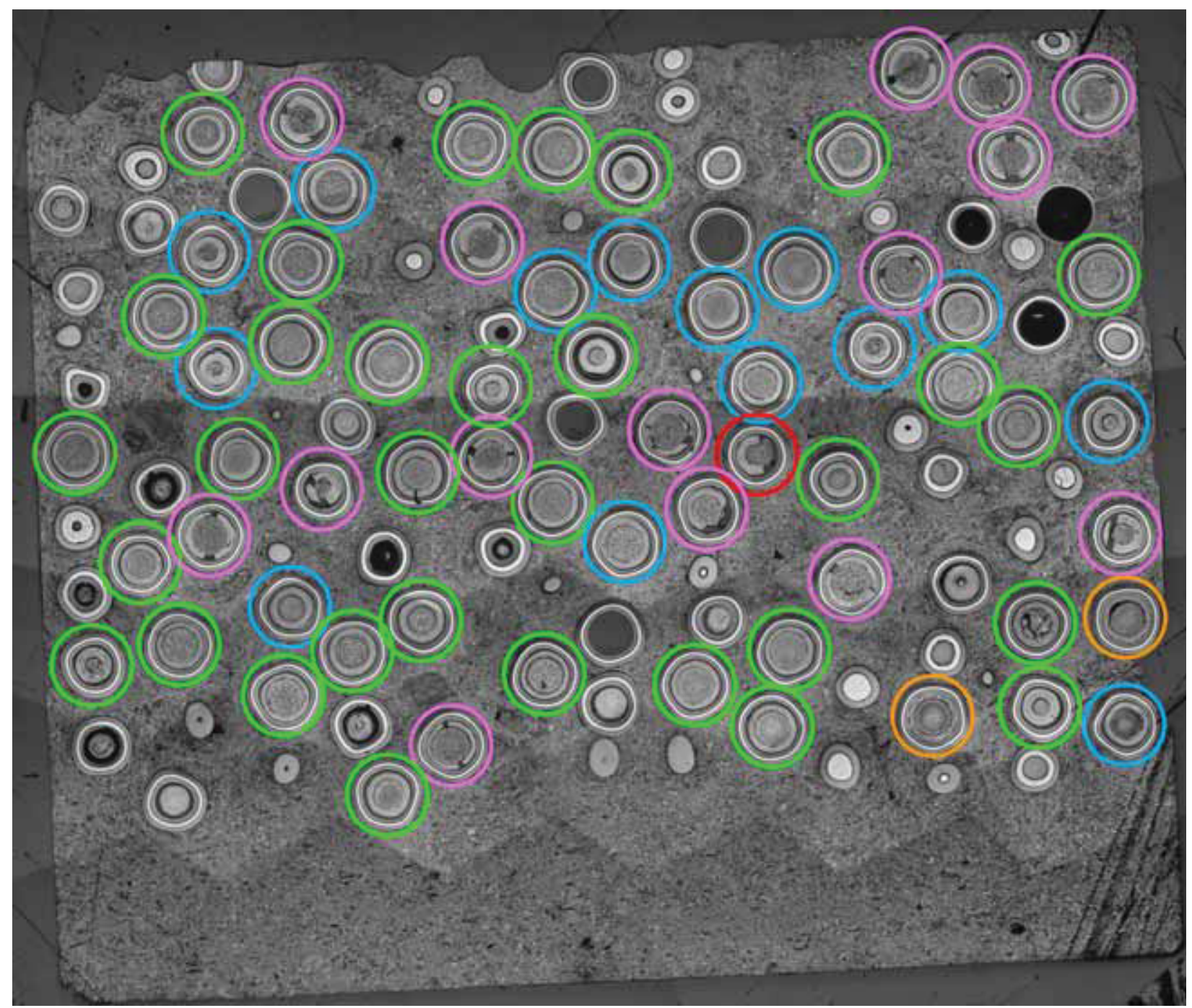

Figure A-6. Spatial distribution of particle morphologies across Compact 2-1-3 (Mount 138T).

A total of 64 intact particles with exposed kernel were characterized as follows:

- $\quad 32$ Type Ai particles (green circles), 50.0\%

- 1 Type Af particle (red circles), 1.6\%

- 14 Type ABi particles (blue circles), 21.9\%

- $\quad 15$ Type ABf particles (magenta circles), 23.4\%

- 2 Type Bi particles (orange circles), 3.1\%

- 0 Type Bf particles (yellow circles), $0.0 \%$. 


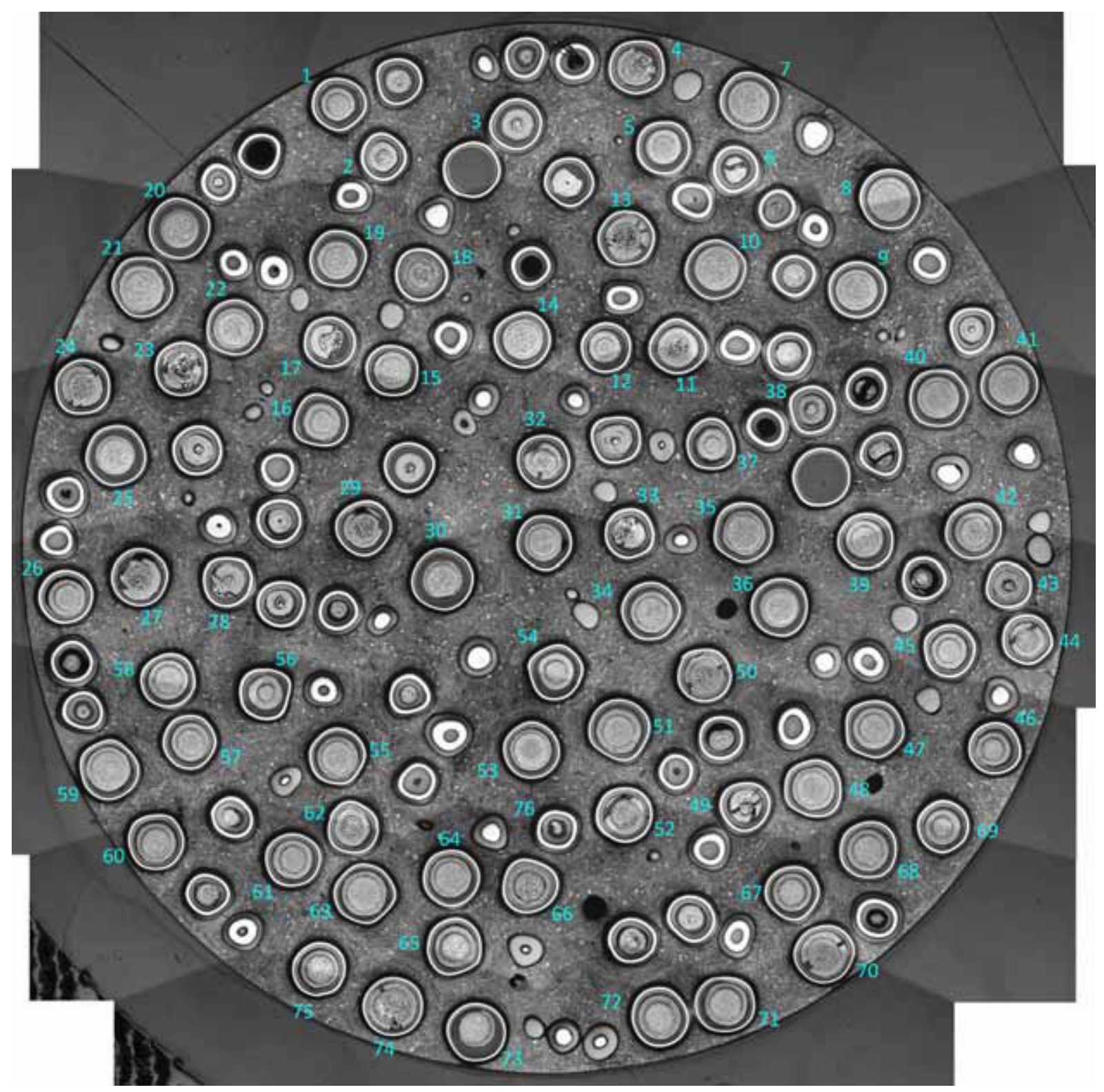

Figure A-7. Transverse cross section through middle of Compact 3-3-3 (Mount 56T) with particle identifiers.

Particle 76 was not sectioned at an elevation exhibiting kernel, but this particle was imaged because it contained a SiC crack. 


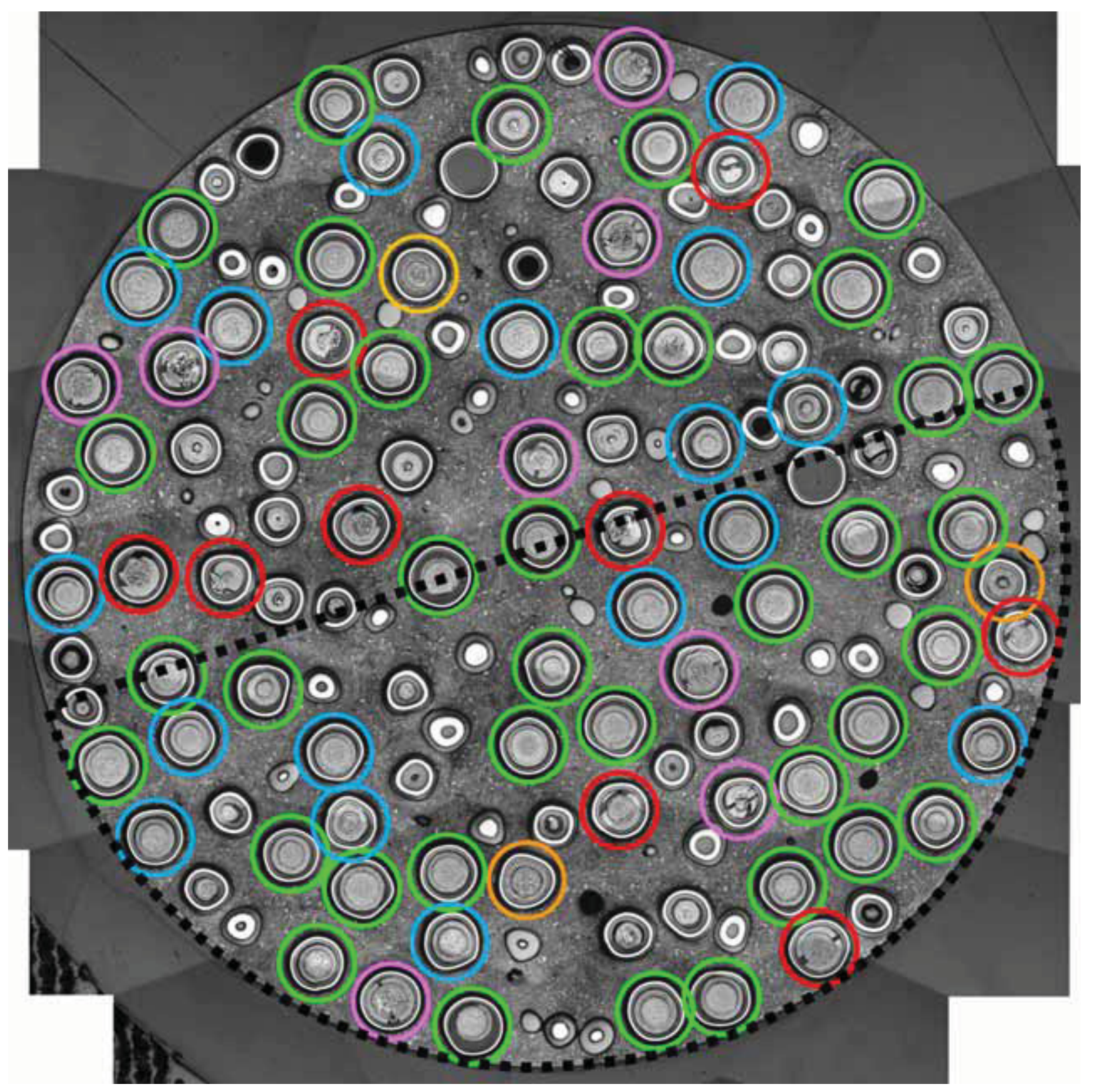

Figure A-8. Spatial distribution of particle morphologies across Compact 3-3-3 (Mount 56T).

The broken outline indicates the approximate position of the accompanying longitudinal sections (Mounts 62T and 68T in Figures A-9 and A-10). A total of 75 intact particles with exposed kernel were characterized as follows:

- 38 Type Ai particles (green circles), 50.7\%

- 9 Type Af particles (red circles), 12.0\%

- $\quad 17$ Type ABi particles (blue circles), 22.7\%

- 8 Type ABf particles (magenta circles), $10.7 \%$

- 3 Type Bi particles (orange circles), $4.0 \%$

- 0 Type Bf particles (yellow circles), $0.0 \%$. 


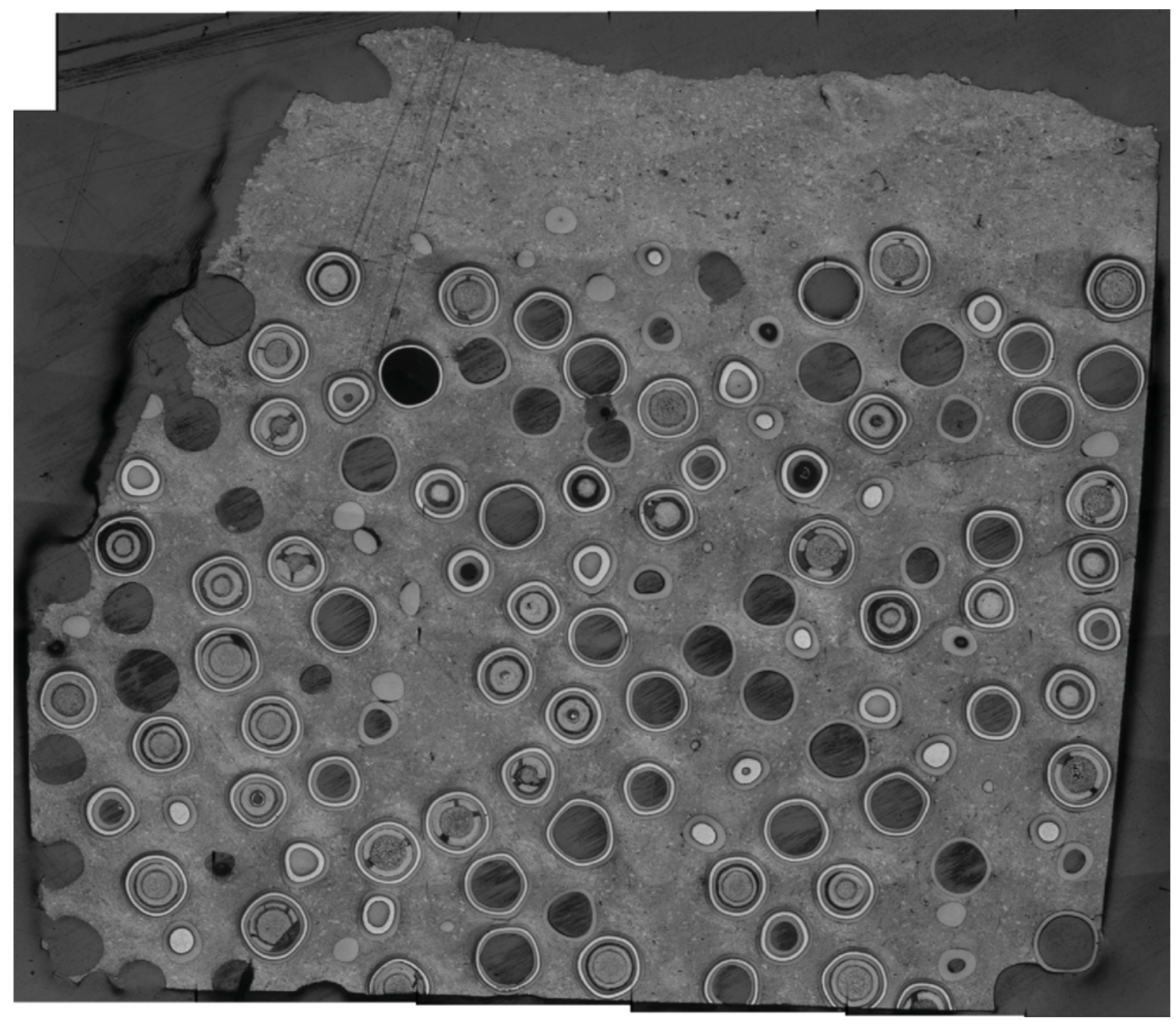

Figure A-9. Longitudinal cross section through top portion of Compact 3-3-3 (Mount 62T).

Images in this montage were taken with the older Leaf Volare camera. Mount 62T has not been incrementally ground and back-potted beyond saw damage. Particle morphologies have not been classified because of the possibility of preferential loss of kernels and buffers. The angled upper left corner in Figure A-9 is because of accidental grinding. This portion of Compact 3-3-3 dropped against a rapidly rotating saw spindle (knurled surface) after the transverse cut. 


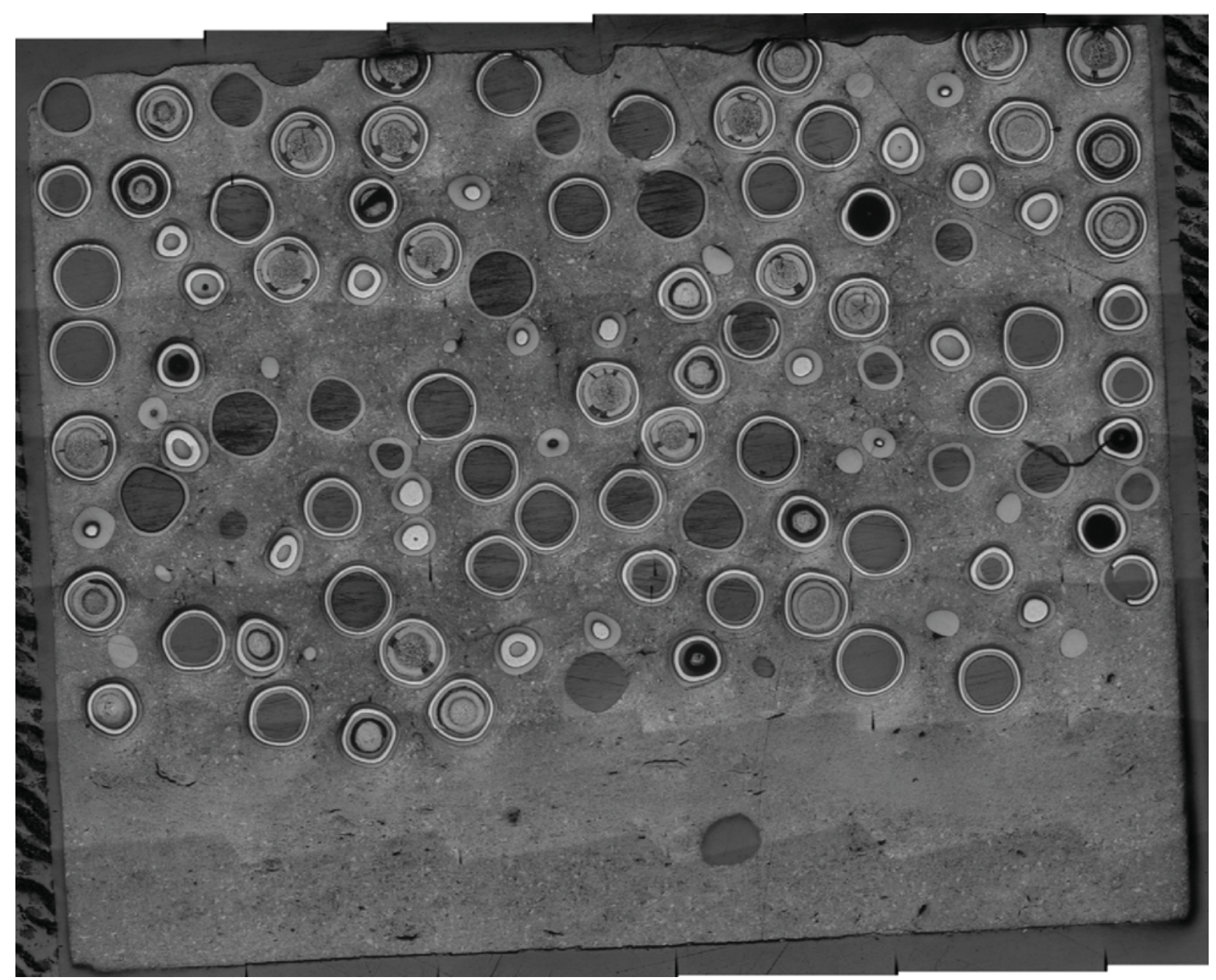

Figure A-10. Longitudinal cross section through bottom part of Compact 3-3-3 (Mount 68T).

Images in this montage were taken with the older Leaf Volare camera. Mount 68T has not been incrementally ground and back-potted beyond saw damage. Particle morphologies have not been classified because of the possibility of preferential loss of kernels and buffers. 


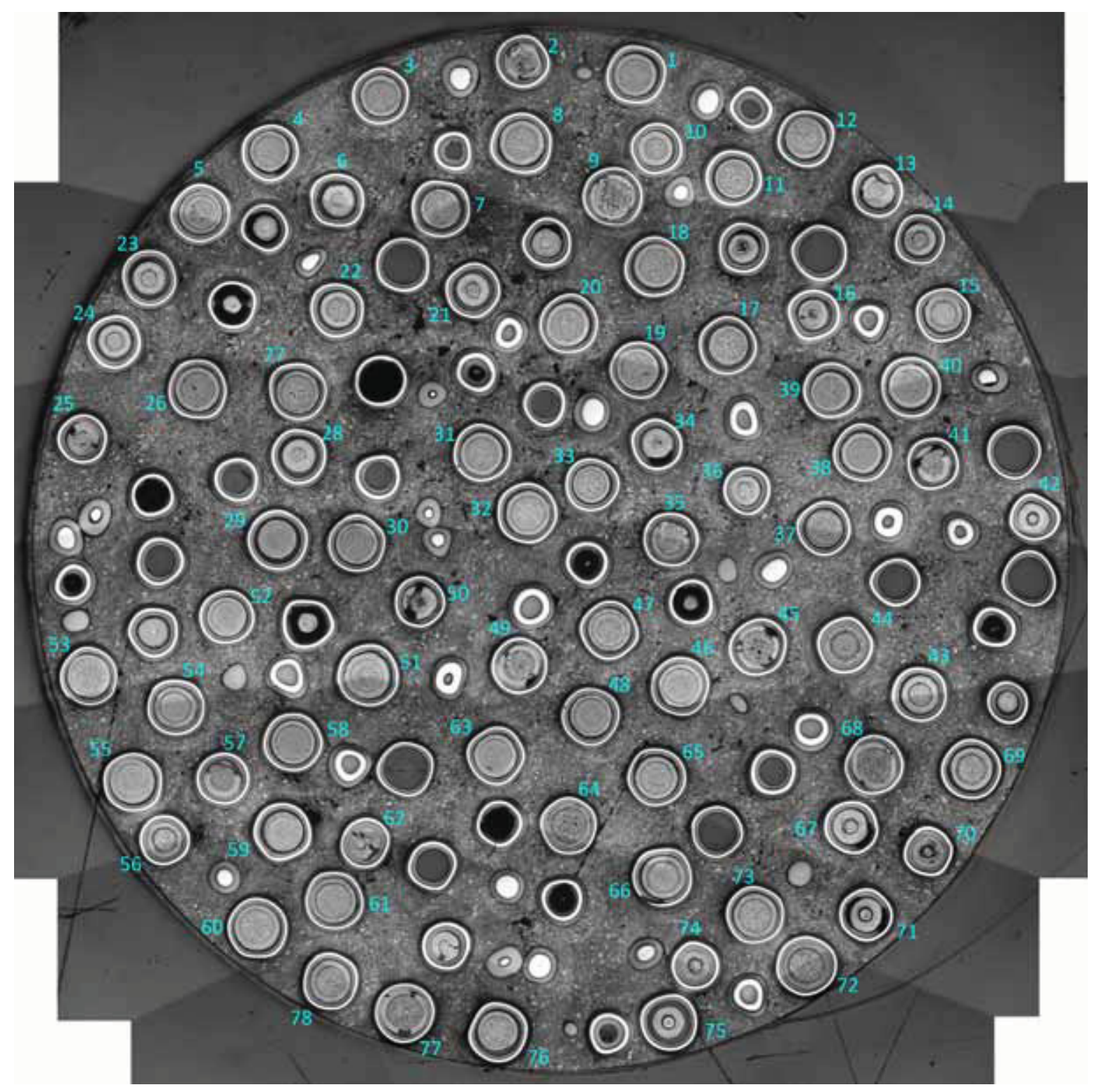

Figure A-11. Transverse cross section through middle of Compact 4-1-3 (Mount 59T) with particle identifiers. 


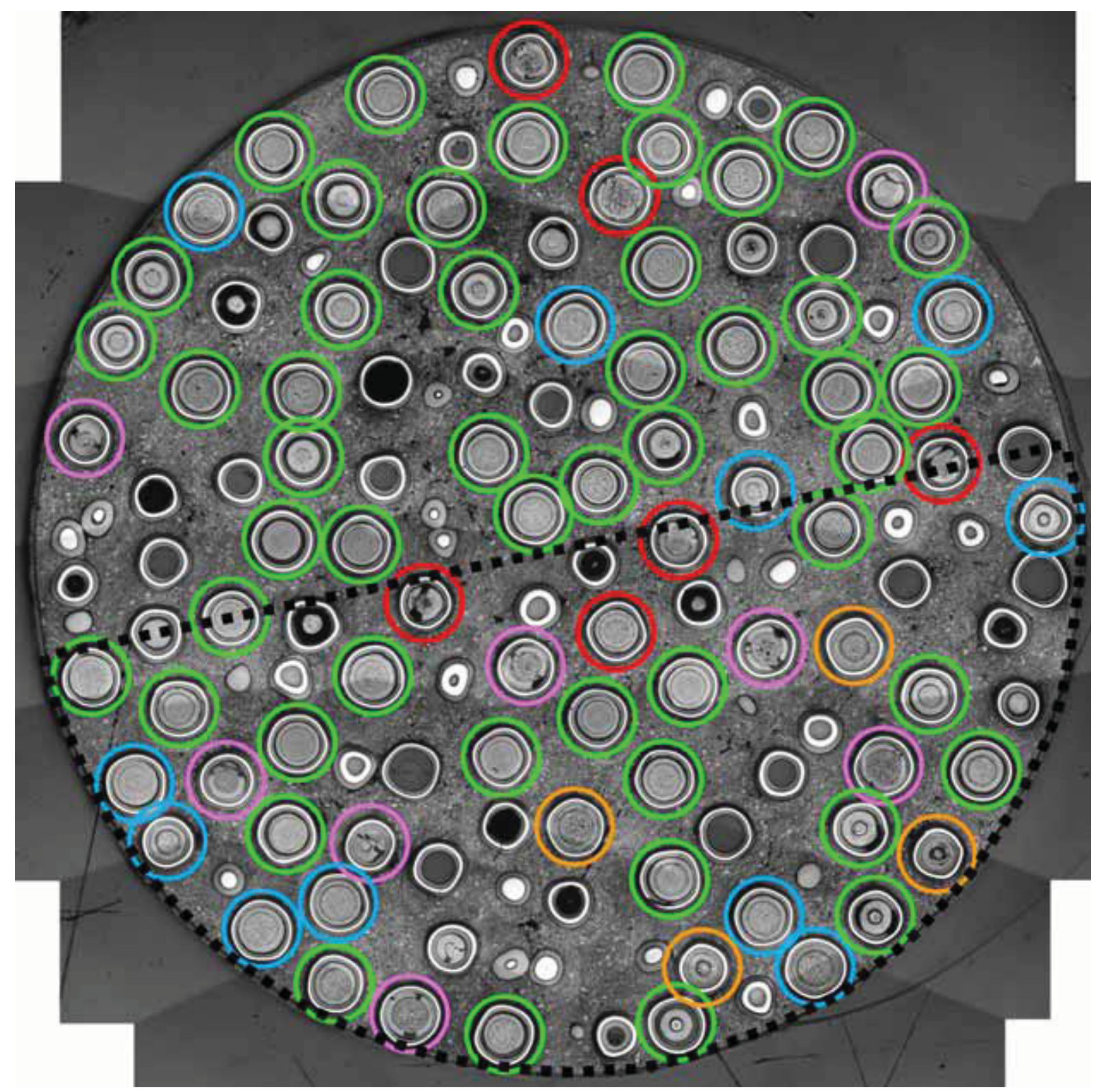

Figure A-12. Spatial distribution of particle morphologies across Compact 4-1-3 (Mount 59T).

The broken outline indicates the approximate position of the accompanying longitudinal sections (both in Mount 64T, Figures A13 through A16). A total of 78 intact particles with exposed kernel were characterized as follows:

- 49 Type Ai particles (green circles), 62.8\%

- 6 Type Af particles (red circles), 7.7\%

- $\quad 11$ Type ABi particles (blue circles), $14.1 \%$

- 8 Type ABf particles (magenta circles), 10.3\%

- 4 Type Bi particles (orange circles), $5.1 \%$

- 0 Type Bf particles (yellow circles), $0.0 \%$. 


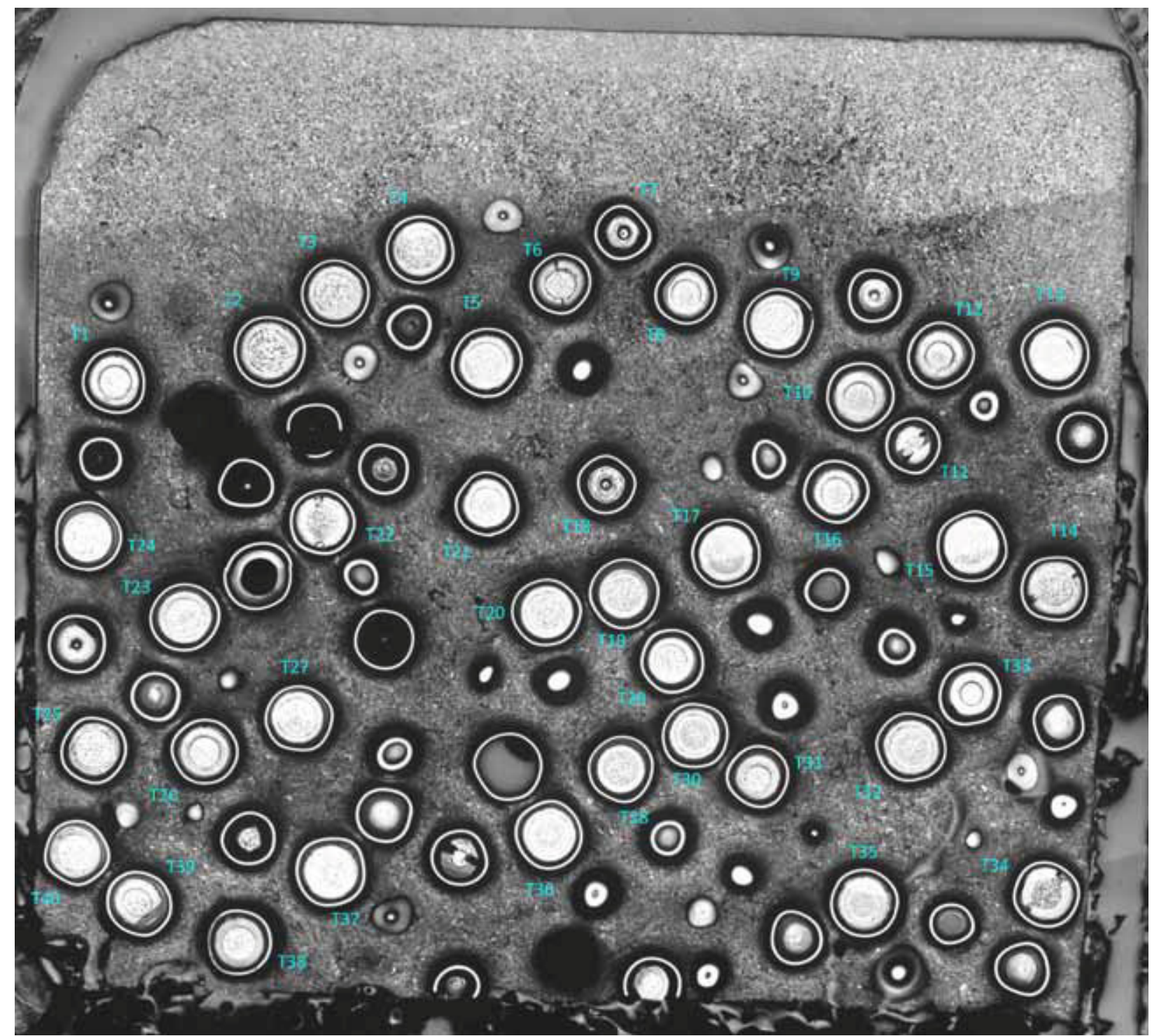

Figure A-13. Longitudinal cross section through top portion of Compact 4-1-3 (in Mount 64T) with particle identifiers. The "T" before the numbers denotes the top longitudinal section. 


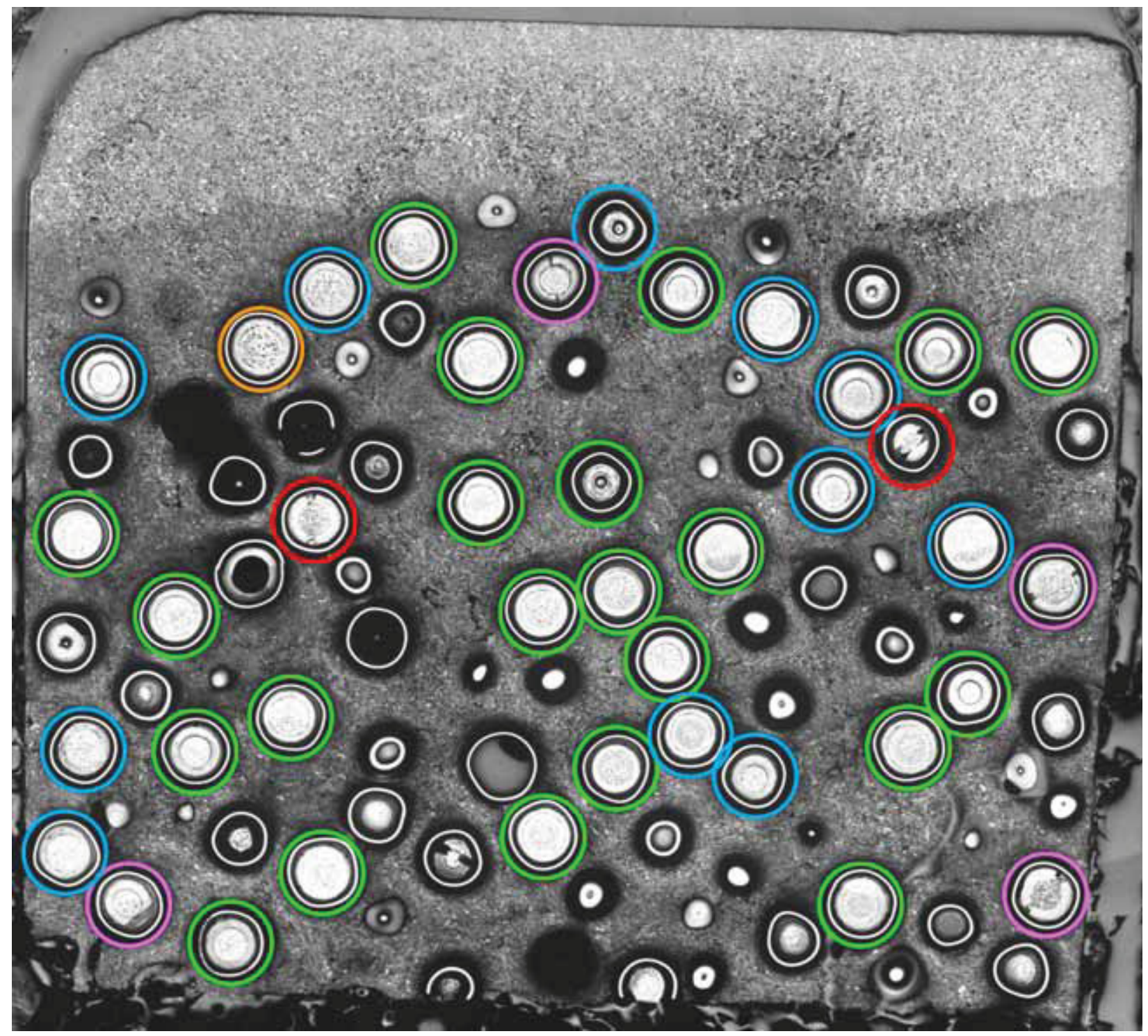

Figure A-14. Spatial distribution of particle morphologies across top portion of Compact 4-1-3 (in Mount 64T).

A total of 40 intact particles with exposed kernel were characterized as follows:

- 22 Type Ai particles (green circles), 55.0\%

- 2 Type Af particles (red circles), 5.0\%

- $\quad 11$ Type ABi particles (blue circles), 27.5\%

- 4 Type ABf particles (magenta circles), 10.0\%

- 1 Type Bi particle (orange circle2), 2.5\%

- 0 Type Bf particles (yellow circles), 0.0\%. 


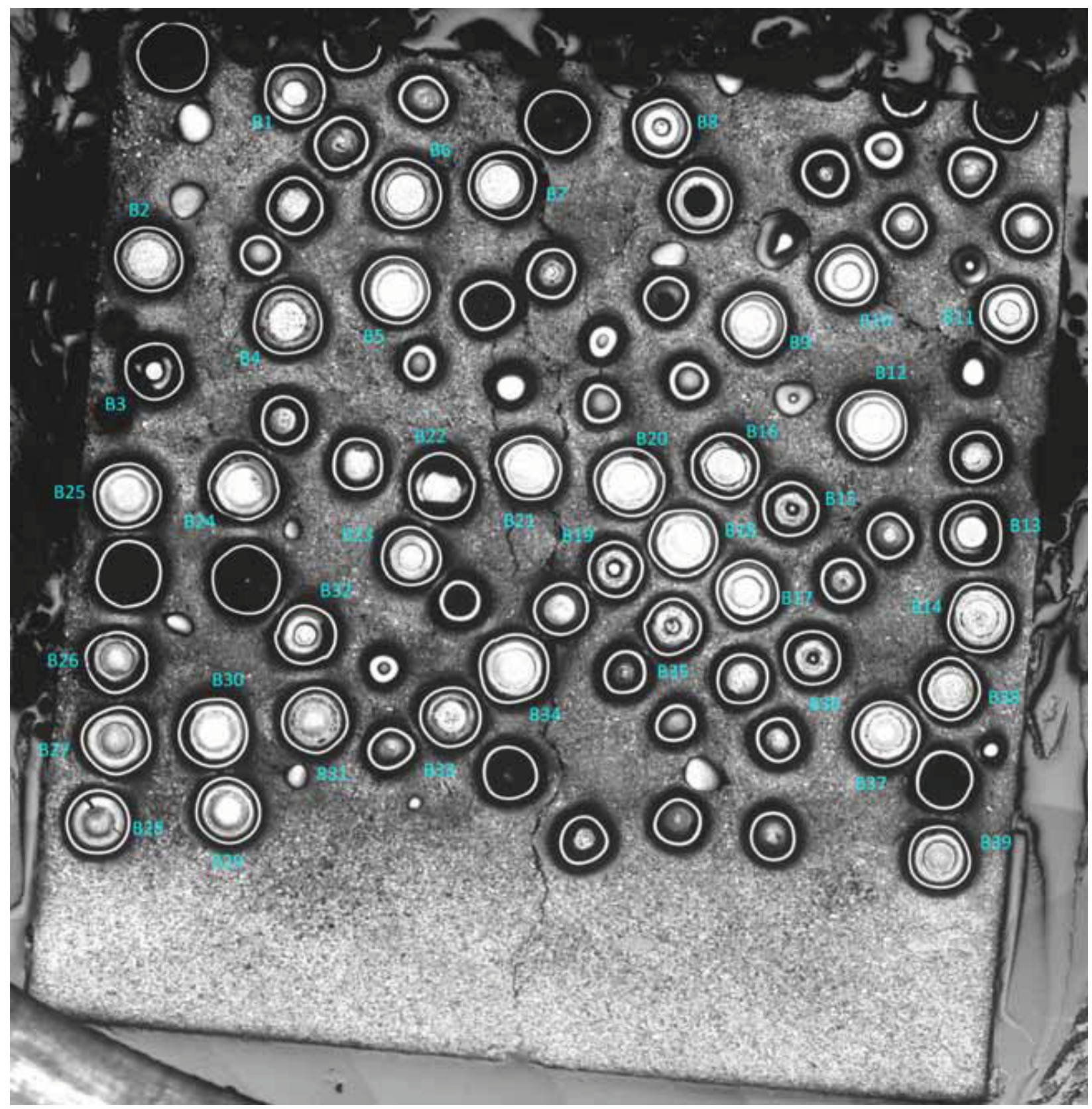

Figure A-15. Longitudinal cross section through bottom portion of Compact 4-1-3 (in Mount 64T) with particle identifiers. The "B" before the numbers denotes the bottom longitudinal section. 


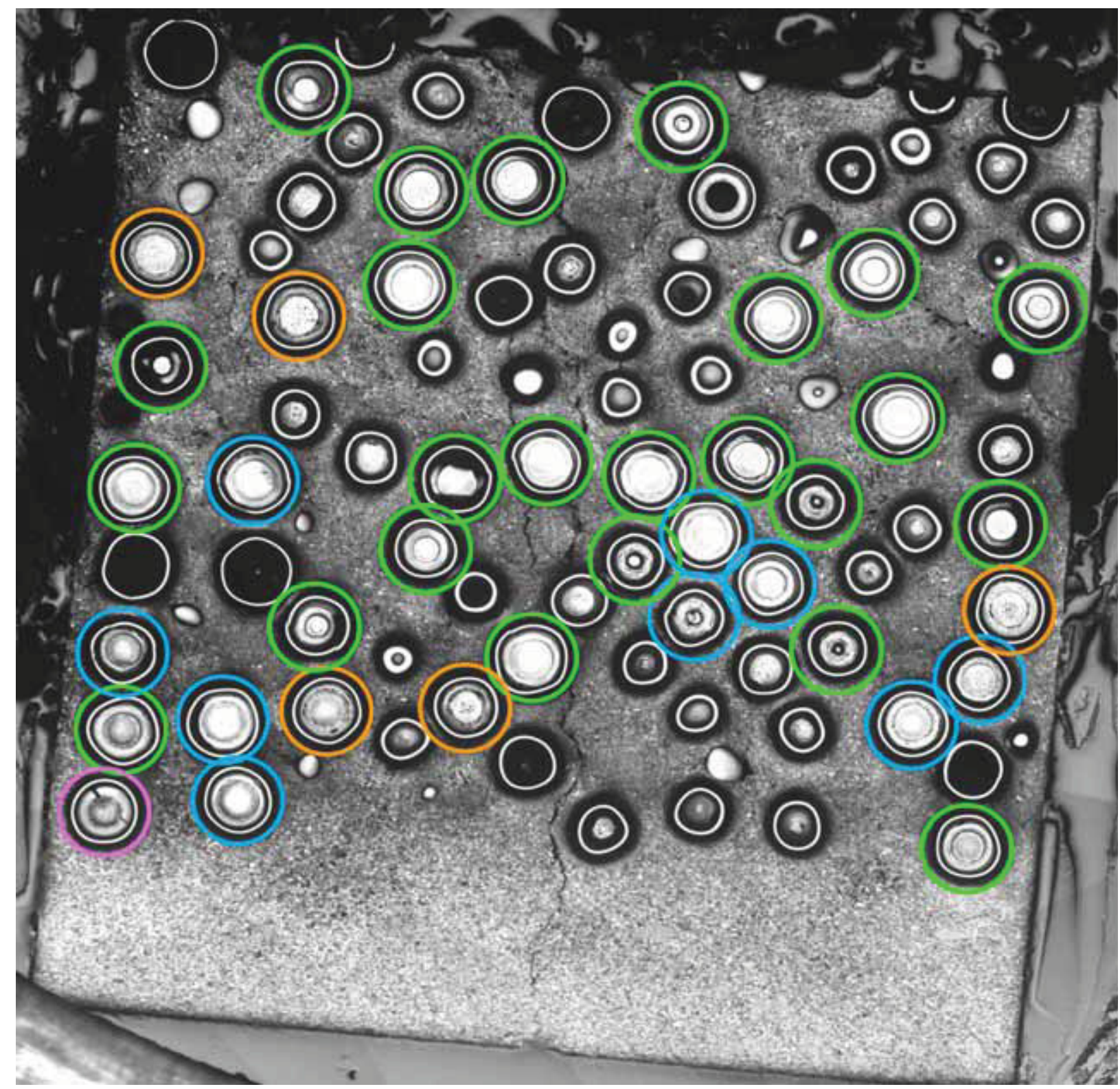

Figure A-16. Spatial distribution of particle morphologies across bottom portion of Compact 4-1-3 (in Mount 64T).

A total of 39 intact particles with exposed kernel were characterized as follows:

- 24 Type Ai particles (green circles), 61.5\%

- 0 Type Af particles (red circles), 0.0\%

- 9 Type ABi particles (blue circles), 23.1\%

- 1 Type ABf particle (magenta circles), 2.6\%

- 5 Type Bi particles (orange circles), 12.8\%

- 0 Type Bf particles (yellow circles), $0.0 \%$. 


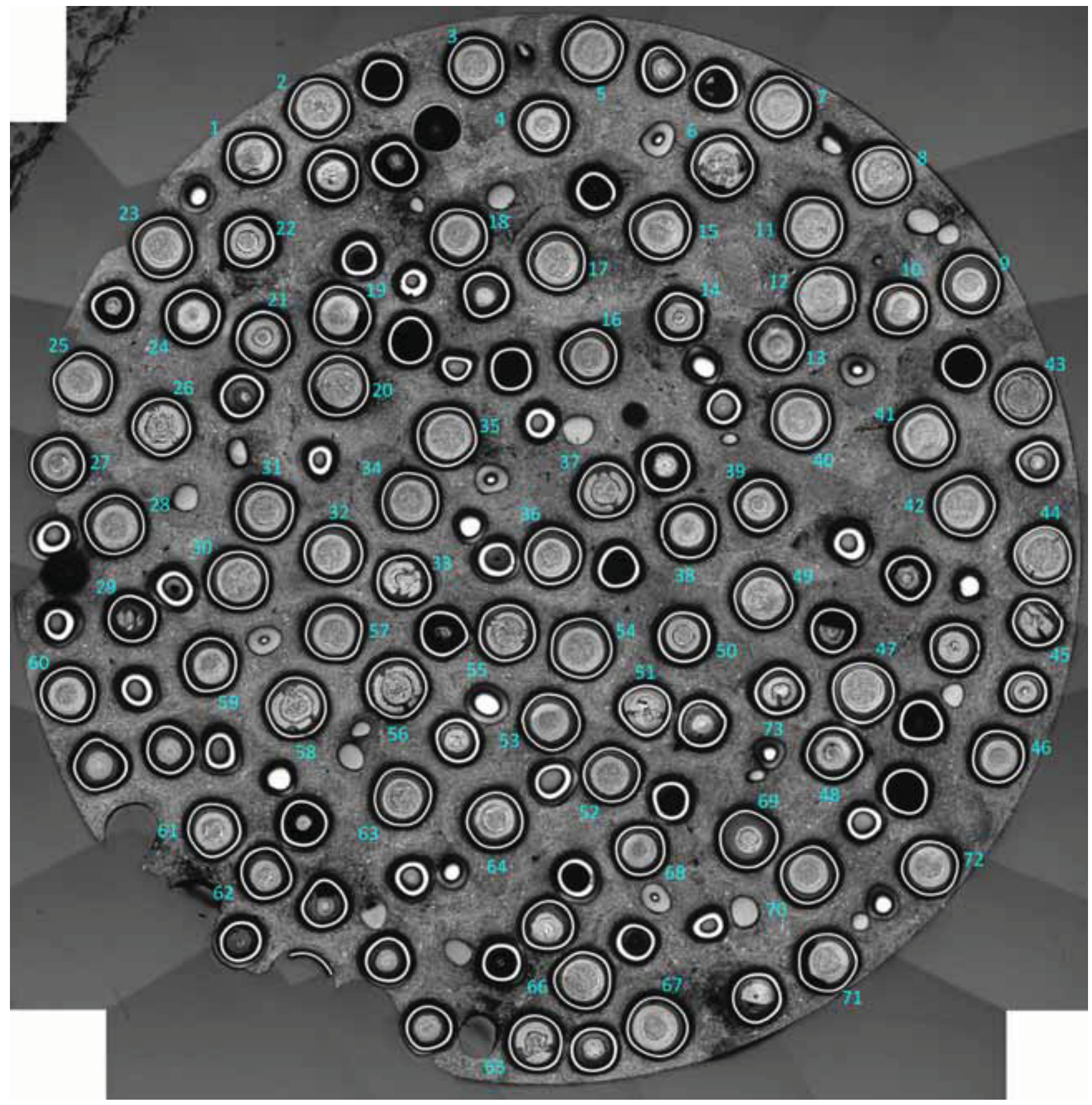

Figure A-17. Transverse cross section through middle of Compact 5-1-2 (Mount 133T) with particle identifiers.

Particle 73 was not sectioned at an elevation exhibiting kernel, but this particle was imaged because it had an unusual buffer fracture. 


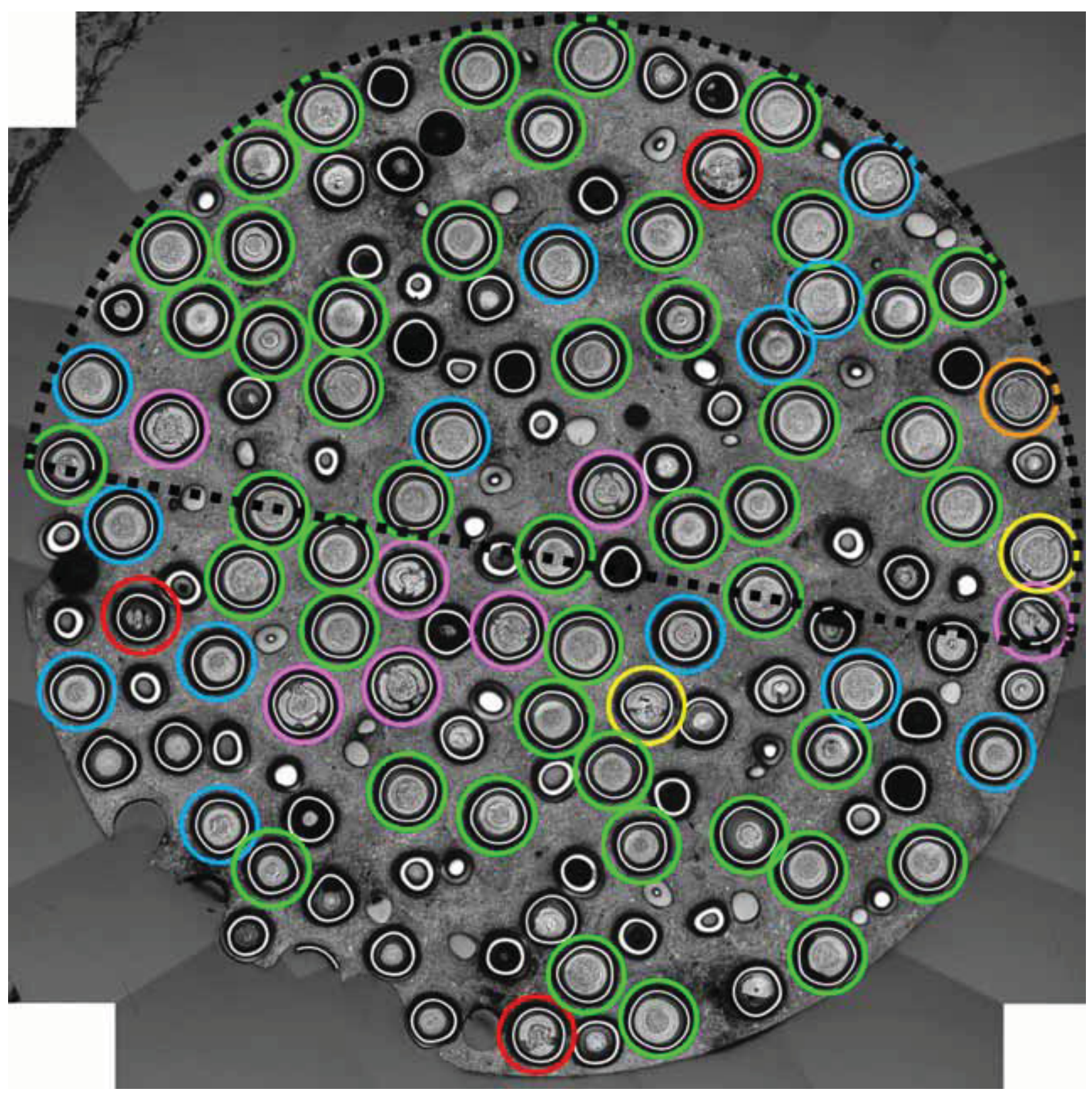

Figure A-18. Spatial distribution of particle morphologies across Compact 5-1-2 (Mount 133T).

The broken outline indicates the approximate position of the accompanying longitudinal sections (Mounts 134T and 135T in Figures A-19 through A-22). A total of 72 intact particles with exposed kernel were characterized as follows:

- 46 Type Ai particles (green circles), 63.9\%

- 3 Type Af particles (red circles), $4.2 \%$

- 13 Type ABi particles (blue circles), 18.1\%

- 7 Type ABf particles (magenta circles), 9.7\%

- 1 Type Bi particle (orange circles), $1.4 \%$

- 2 Type Bf particles (yellow circles), $2.8 \%$. 


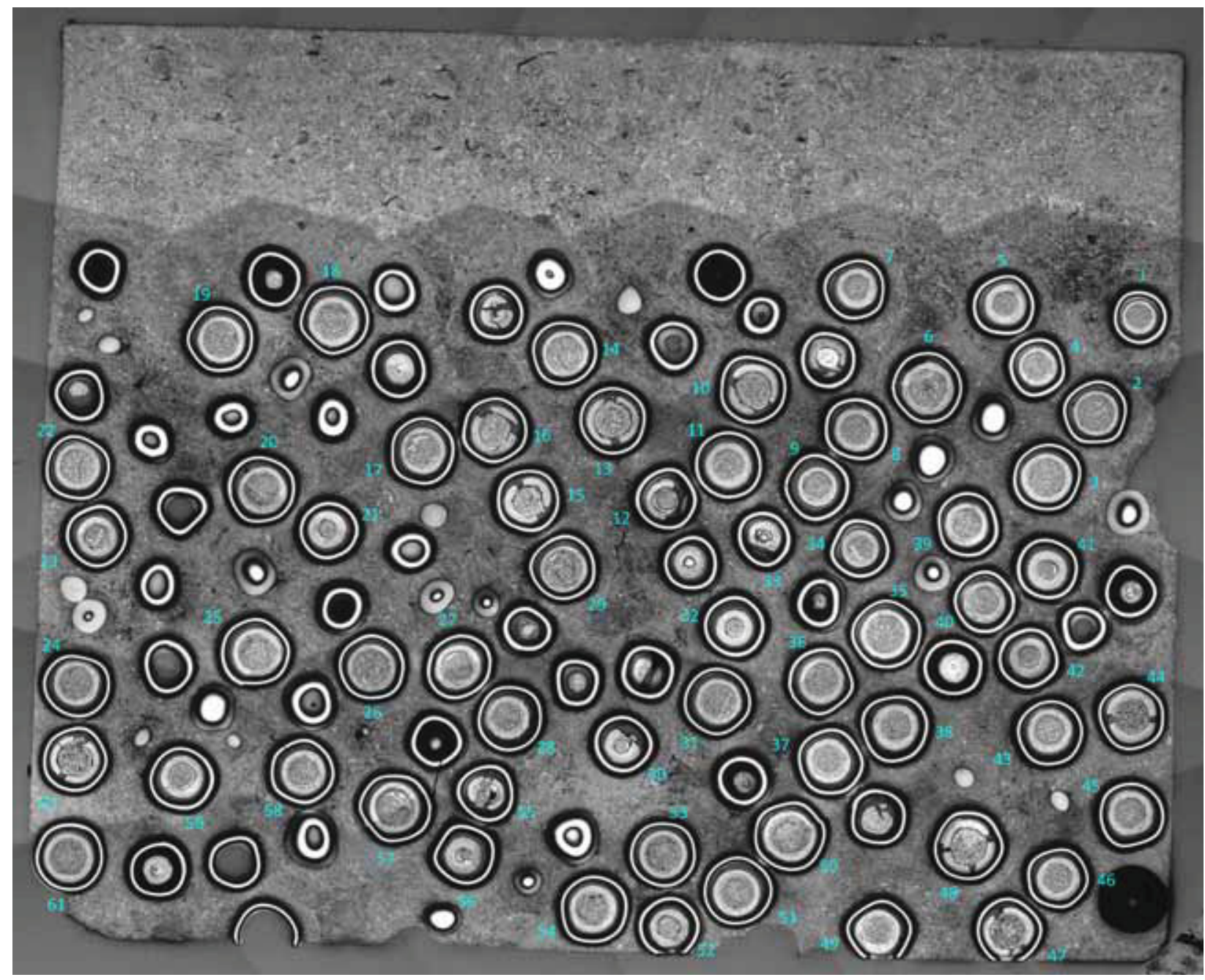

Figure A-19. Longitudinal cross section through top portion of Compact 5-1-2 (Mount 134T) with particle identifiers. 


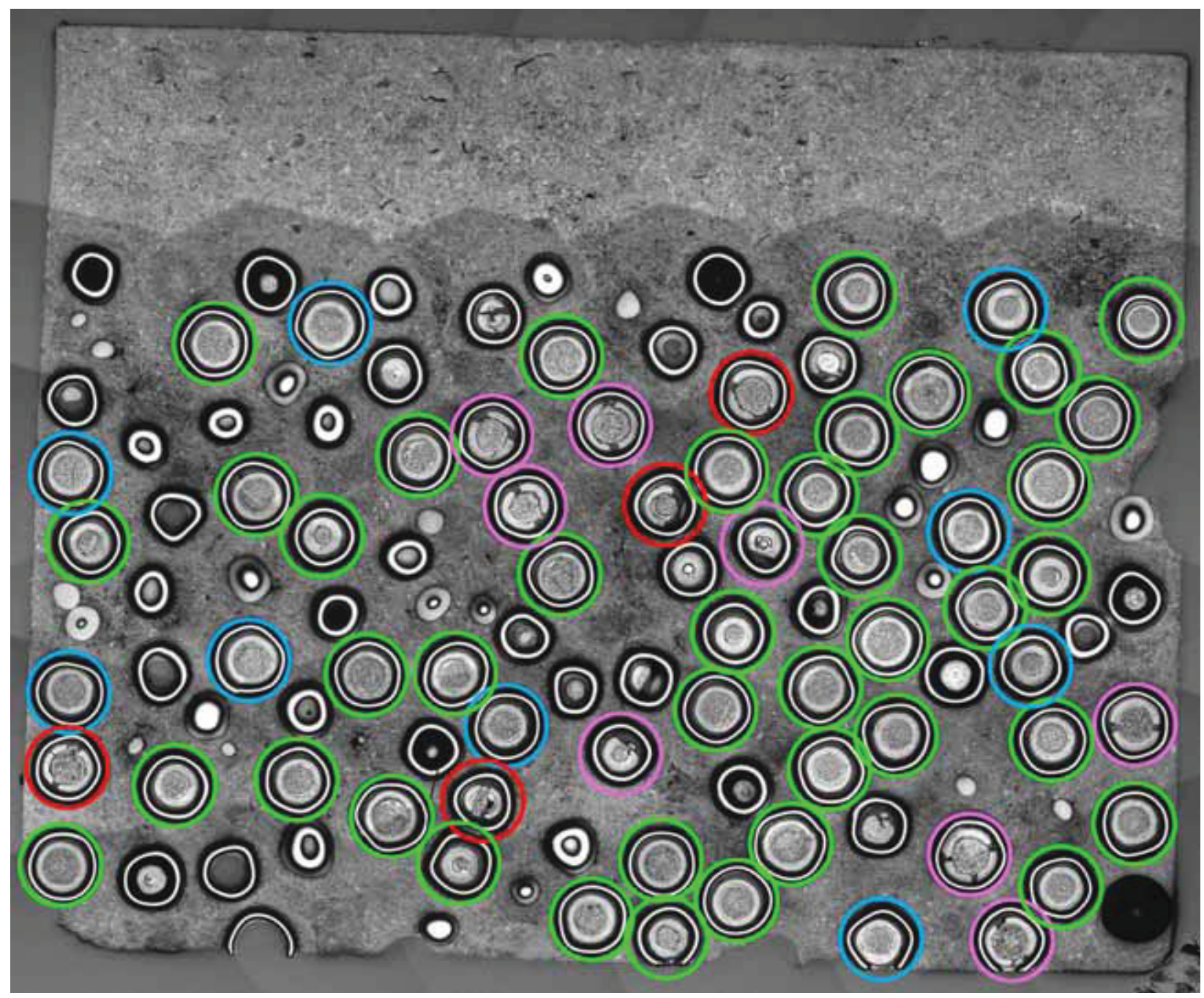

Figure A-20. Spatial distribution of particle morphologies across top portion of Compact 5-1-2 (Mount 134T).

A total of 61 intact particles with exposed kernel were characterized as follows:

- 40 Type Ai particles (green circles), 65.6\%

- 4 Type Af particles (red circles), 6.6\%

- 9 Type ABi particles (blue circles), $14.8 \%$

- 8 Type ABf particles (magenta circles), $13.1 \%$

- 0 Type Bi particles (orange circles), $0.0 \%$

- 0 Type Bf particles (yellow circles), $0.0 \%$. 


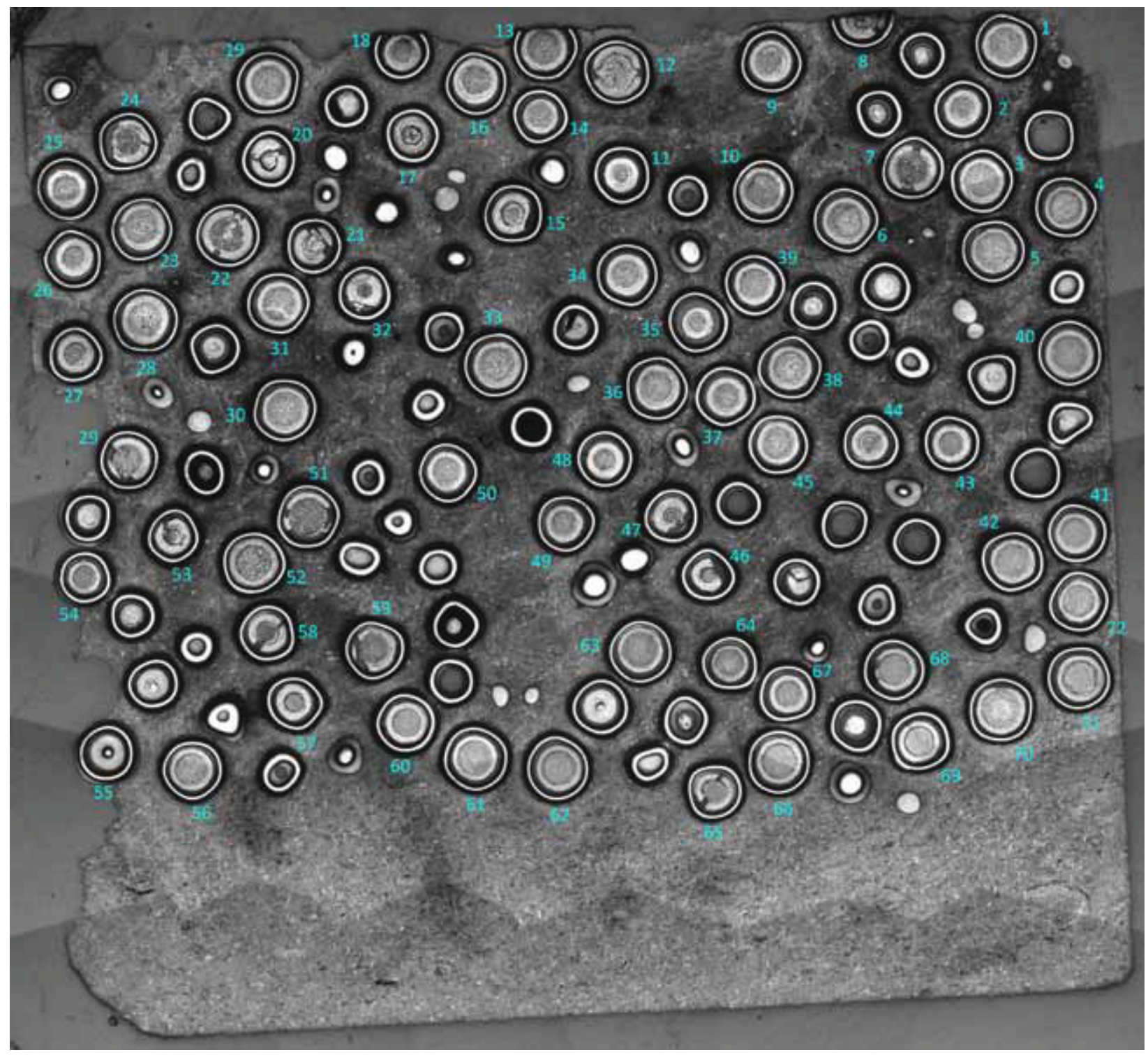

Figure A-21. Longitudinal cross section through bottom portion of Compact 5-1-2 (Mount 135T) with particle identifiers. 




Figure A-22. Spatial distribution of particle morphologies across bottom portion of Compact 5-1-2 (Mount 135T).

A total of 72 intact particles with exposed kernel were characterized as follows:

- 37 Type Ai particles (green circles), 51.4\%

- 7 Type Af particles (red circles), 9.7\%

- $\quad 17$ Type ABi particles (blue circles), 23.6\%

- 9 Type ABf particles (magenta circles), 12.5\%

- 2 Type Bi particles (orange circles), 2.8\%

- $\quad 0$ Type Bf particles (yellow circles), $0.0 \%$. 


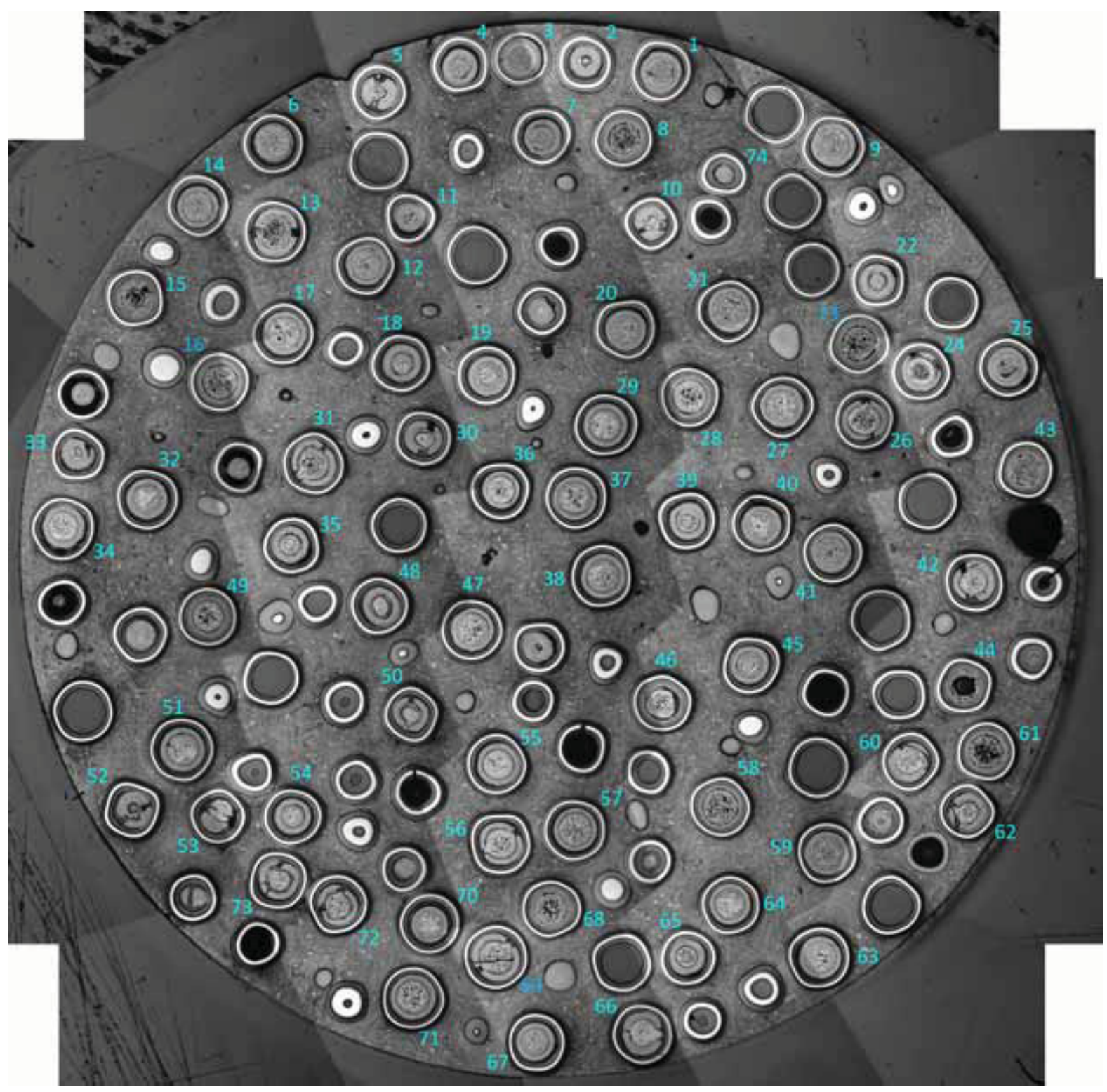

Figure A-23. Transverse cross section through middle of Compact 6-3-3 (Mount 57T) with particle identifiers.

Particle 74 was not sectioned at an elevation exhibiting kernel, but this particle was imaged because it contained a deep scratch in the $\mathrm{SiC}$ layer. 


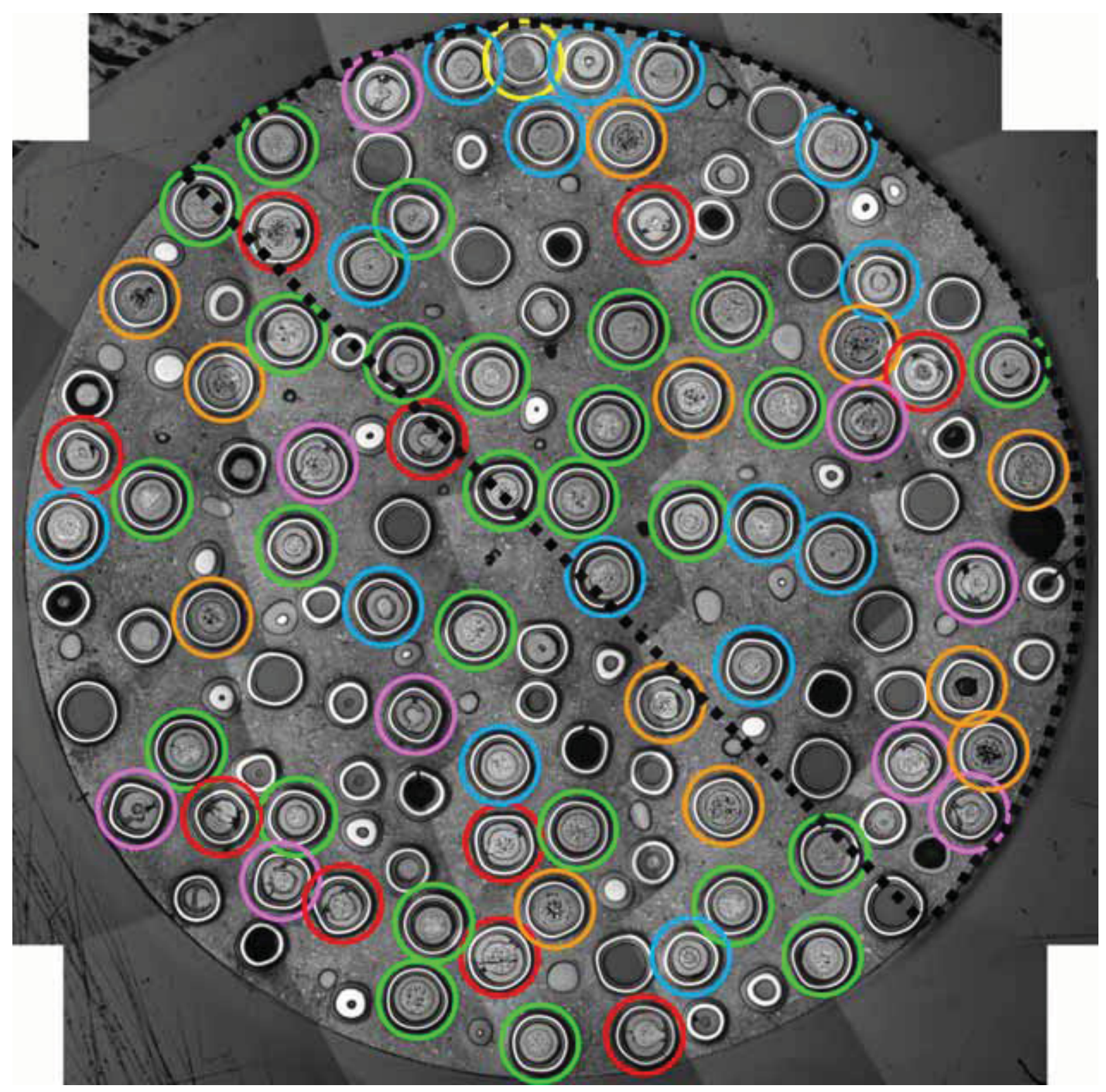

Figure A-24. Spatial distribution of particle morphologies across Compact 6-3-3 (Mount 57T).

The broken outline indicates the approximate position of the accompanying longitudinal sections (both in Mount 67T, Figures A-25 through A-28). A total of 73 intact particles with exposed kernel were characterized as follows:

- 26 Type Ai particles (green circles), 35.6\%

- 10 Type Af particles (red circles), $13.7 \%$

- 15 Type ABi particles (blue circles), 20.5\%

- 9 Type ABf particles (magenta circles), $12.3 \%$

- 12 Type Bi particles (orange circles), 16.4\%

- 1 Type Bf particle (yellow circles), 1.4\%. 


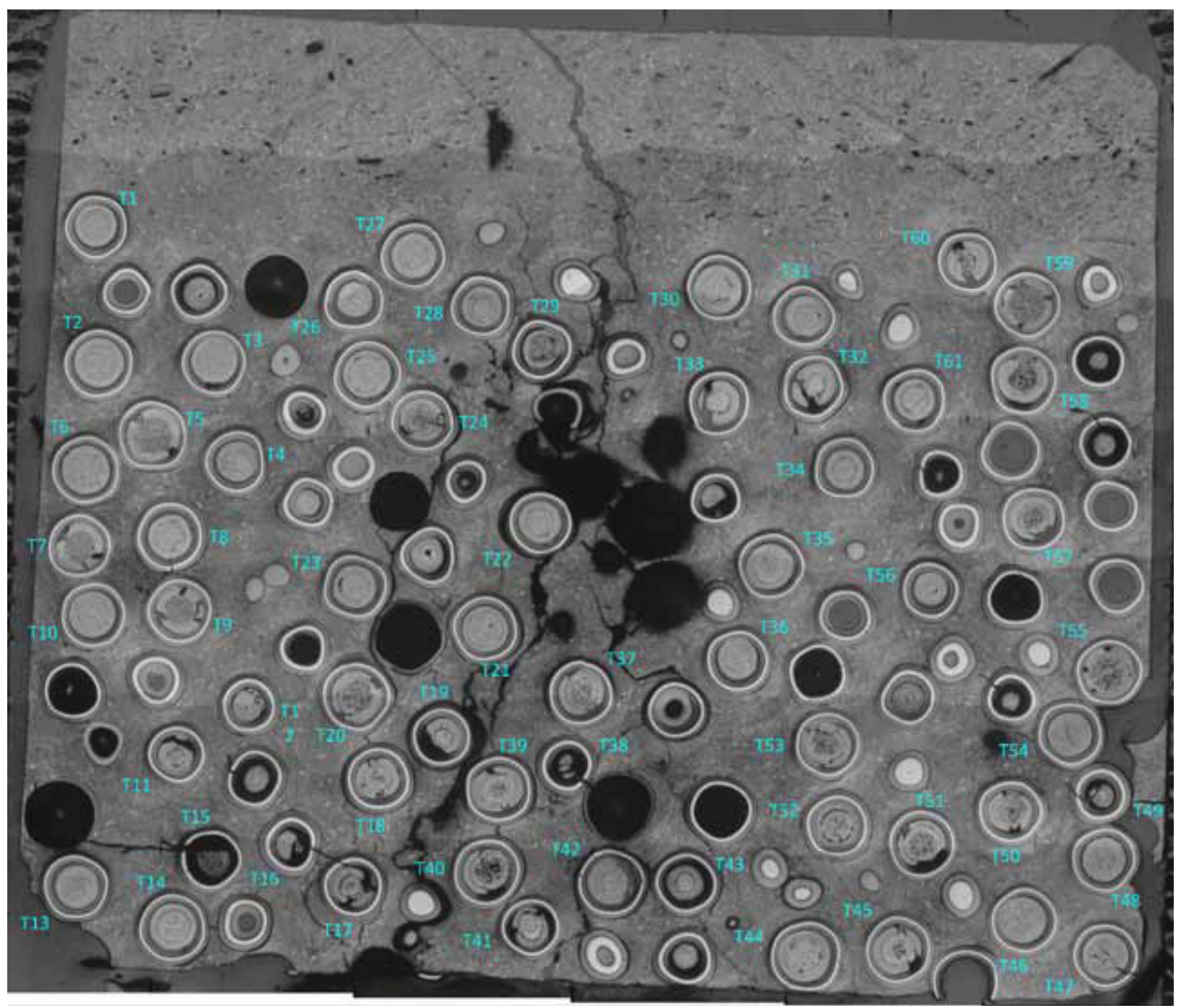

Figure A-25. Longitudinal cross section through top portion of Compact 6-3-3 (in Mount 67T) with particle identifiers. The " $T$ " before the numbers denotes the top longitudinal section. This portion of the compact fractured during sawing. 


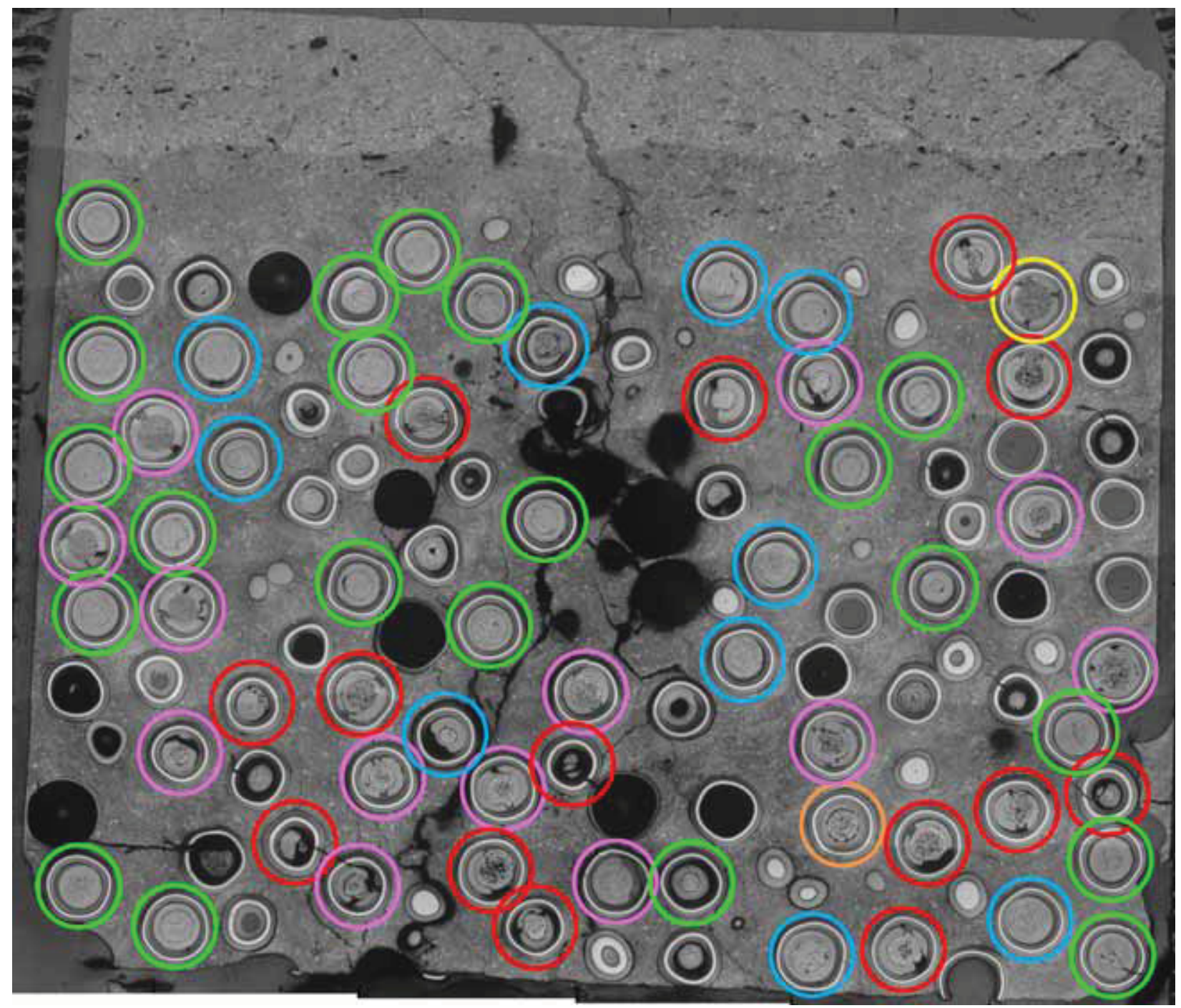

Figure A-26. Spatial distribution of particle morphologies across top portion of Compact 6-3-3 (in Mount 67T).

In this mount 60 of 61 intact particles with exposed kernel were characterized as follows, but too much buffer was missing in Particle T15 for definite classification:

- 21 Type Ai particles (green circles), 35.0\%

- 14 Type Af particles (red circles), 23.3\%

- $\quad 10$ Type ABi particles (blue circles), 16.7\%

- 13 Type ABf particles (magenta circles), 21.7\%

- 1 Type Bi particle (orange circles), 1.7\%

- 1 Type Bf particle (yellow circles), 1.7\%. 


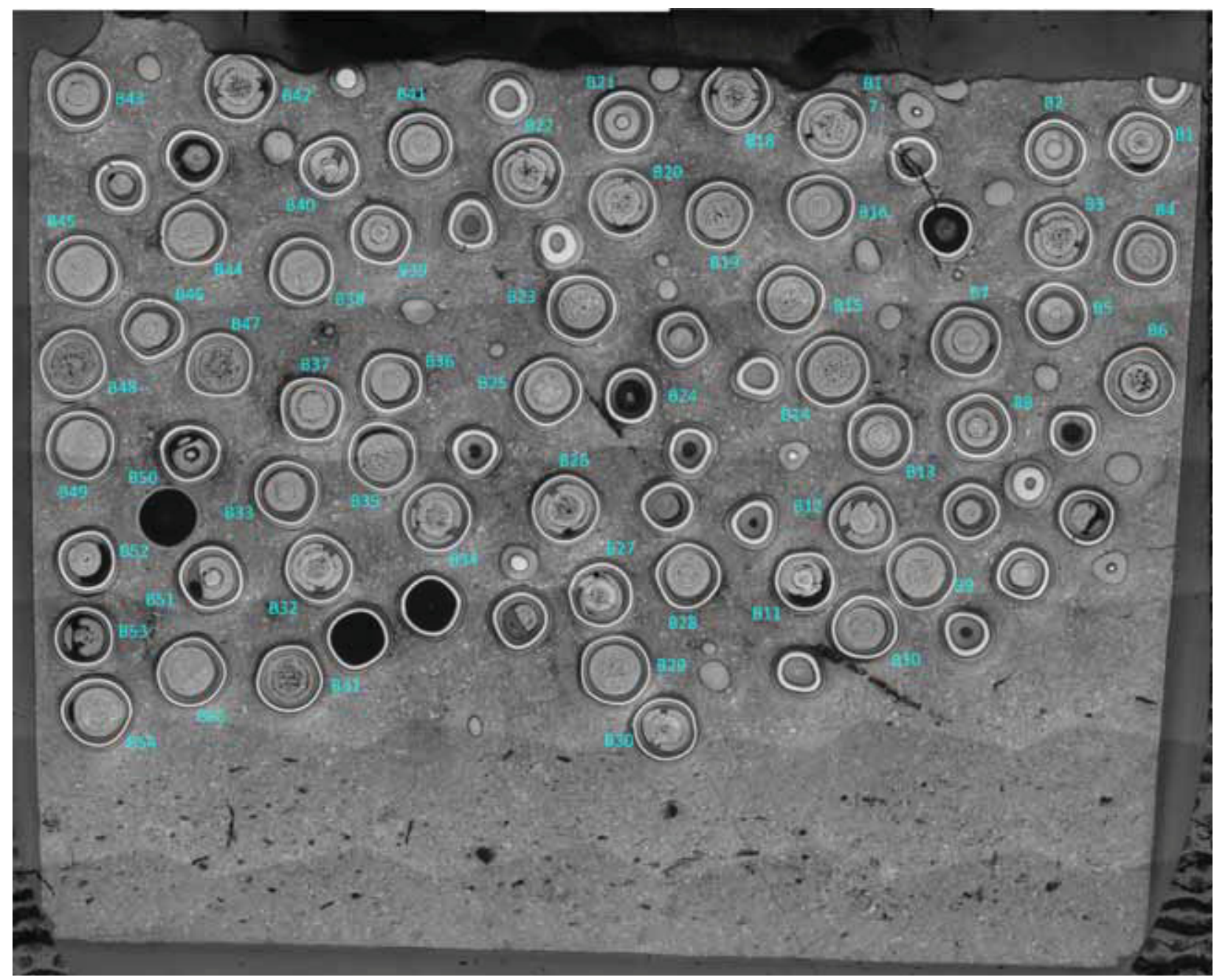

Figure A-27. Longitudinal cross section through bottom portion of Compact 6-3-3 (in Mount 67T) with particle identifiers. The "B" before the numbers denotes the bottom longitudinal section. 


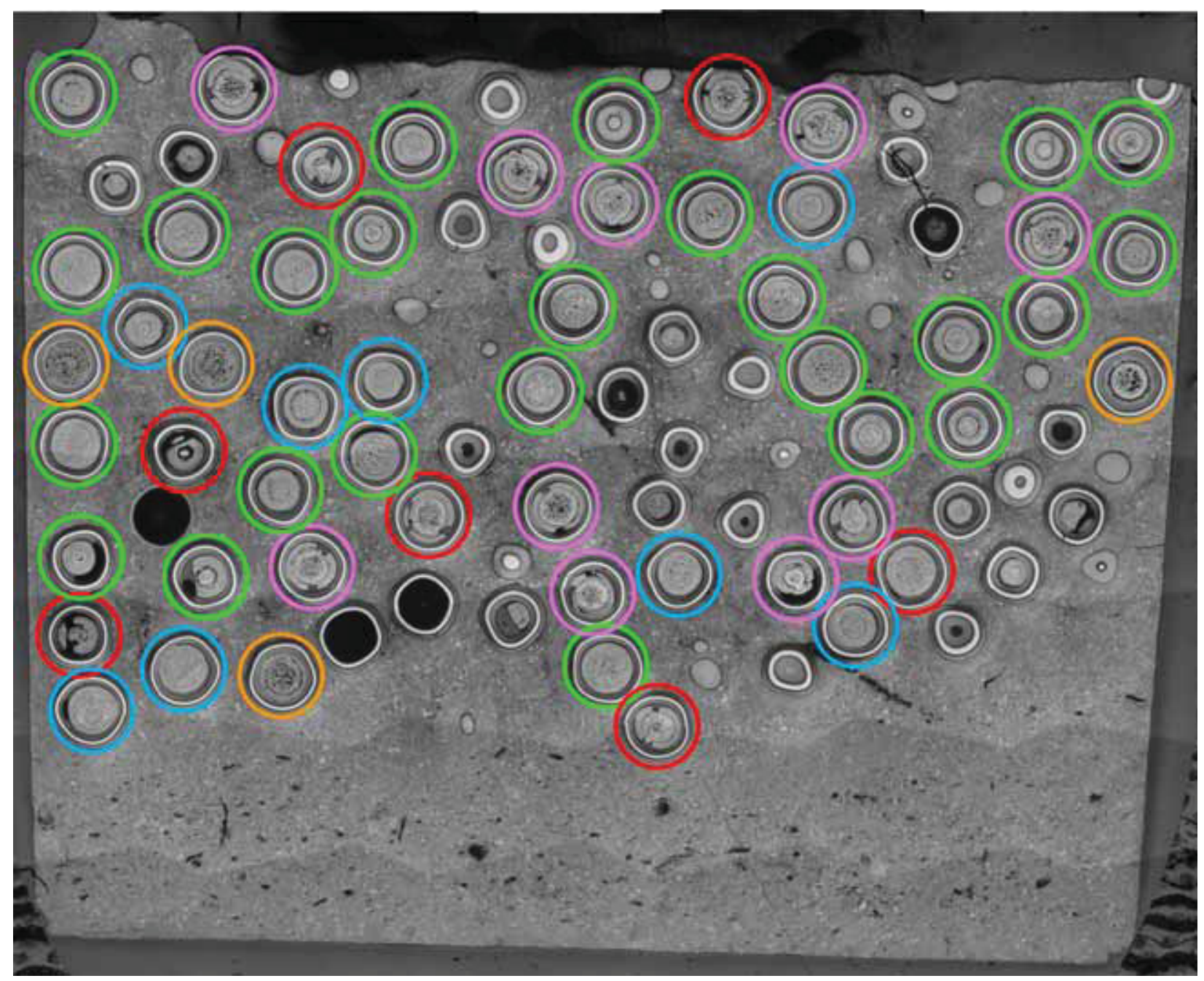

Figure A-28. Spatial distribution of particle morphologies across bottom portion of Compact 6-3-3 (in Mount 67T).

In this mount 54 of 55 intact particles with exposed kernel were characterized as follows, but too much buffer was missing in Particle B24 for definite classification:

- 25 Type Ai particles (green circles), 46.3\%

- 7 Type Af particles (red circles), 13.0\%

- 8 Type ABi particles (blue circles), $14.8 \%$

- $\quad 10$ Type ABf particles (magenta circles), $18.5 \%$

- 4 Type Bi particles (orange circles), 7.4\%

- $\quad 0$ Type Bf particles (yellow circles), $0.0 \%$. 